\title{
Gradient expansion approach to multiple-band Fermi liquids
}

\author{
Ryuichi Shindou, ${ }^{1, *}$ and Leon Balents ${ }^{1}$ \\ ${ }^{1}$ Department of Physics, University of California, Santa Barbara, California 93106, USA
}

(Dated: today)

\begin{abstract}
Promoted by the recent progress of Berry phase physics in spin galvanomagnetic communities, we develop a systematic derivation of the reduced Keldysh equation (RKE) which captures the low-energy dynamics of quasi-particles constrained within doubly degenerate bands forming a single Fermi surface. The derivation begins with the Keldysh equation for a quite general multiple-band interacting Fermi systems, which is originally an $N_{b}$ by $N_{b}$ matrix-formed integral (or infinite-order differential) equation, with $N_{b}$ being the total number of bands. To derive the RKE for quasi-particle on a Fermi surface in question, we project out the fully occupied/empty band degrees of freedom perturbatively in the gradient expansion, whose coupling constant measures how a system is disequilibrated. As for the electron-electron interactions, however, we only employ the so-called adiabatic assumption of the Fermi liquid theory, so that the electron correlations effect onto the adiabatic transport of quasi-particles, i.e. the hermitian (real) part of the self-energy, is taken into account in an unbiased manner. The RKE thus derived becomes an $S U(2)$ covariant differential equation and treats the spin and charge degrees of freedom on an equal footing. Namely, the quasi-particle spin precessions due to the non-abelian gauge fields are automatically encoded into its covariant derivatives. When further solved in favor of spectral functions, this covariant differential equation also suggests that quasi-particles on a doubly degenerate Fermi surface acquire spin-selective Berry curvature corrections under the applied electromagnetic fields. This theoretical observation gives us some hints of possible experimental methodology for measuring the $S U(2)$ Berry's curvatures by spin-resolved photoemission experiments. Due to the non-trivial frequency dependence of (the hermitian part of) self energy, our RKE is composed of Berry's curvatures in the $d+1$ dual space, i.e. $k-\omega$ space, so that the dual electric field is already introduced. To provide a simple way to understand this "temporal" component of the $U(1)$ Berry's curvature, we also provide the dual analogue of the Ampere law, where the "spatial" rotation of the electric field in combination with the "temporal" derivative of the well-known magnetic component are determined by the $U(1)$ magnetic monopole "current".
\end{abstract}

PACS numbers: 71.10.Ay, 71.27.+a, 79.60.-i, 72.15.Gd

\section{INTRODUCTION}

Gauge fields often appear in effective low energy theories in condensed matter physics, whenever the system's low energy manifold is restricted by some "local" constraints $\stackrel{1}{1}$. Classic examples include doped MottHubbard insulators, two dimensional electron gases in the fractional quantum Hall regime, low-dimensional quantum spin systems and highly frustrated magnets.

In these "strong-coupling" problems, the constraints are implemented locally in real space. For example, the on-site Coulomb interaction in the Hubbard model or t-J model forbids double occupancy on every site. In strong magnetic fields, the wavefunction for electrons in a two dimensional electron gas must be annihilated by the Landau level lowering operator, which is a differential operator defined locally for each value of the twodimensional coordinate. In the quantum dimer model, spins are presumed to be bound into singlets which cover each site exactly once. In many cases, these local constraints take the form of a version of "Gauss' law" imposed on suitably introduced "electromagnetic field" and "charge" variables. Due to the Gauss' law constraint, the low energy effective theory for such systems becomes mathematically equivalent to a form of (usually compact) quantum electro-dynamics $1,3,4$.
Another kind of constrained system occurs in the weak coupling region, such as in a Fermi liquid (FL), where the states at the fermi surface are supposed to be well separated from the other fully occupied/empty bands by a sufficiently large (direct) band gap. The low energy manifold is thus spanned only by the Bloch states of those conduction bands forming this Fermi surface. Even in the presence of strong electron correlations, we may still assume that the low energy Hilbert space is spanned only by those quasi-particle excitations constrained within a Fermi surface, as far as electron-electron interactions can be introduced adiabatically in comparison with the direct band gap.

In most of the literature, Fermi liquids are assumed to be well described, by ignoring electron correlations completely. In such a non-interacting case, the projection to a single low-energy band or degenerate bands has been studied extensively $5.6,7,8,9.10$, and yields an effective equation of motion (EOM) for the conduction electrons in this band, say the $\alpha$-th band, moving under the influence of external electric field $\mathbf{e}$ and magnetic field $\mathbf{b}$. Such an EOM contains an dual magnetic field $\mathcal{B}^{\alpha}$, which acts on a quasi-particle like a Lorentz force in $k$-space:

$$
\frac{d R}{d T}=\mathbf{z}^{\dagger}\left\{\hat{\mathbf{v}}_{\alpha}+\mathcal{B}^{\alpha} \times \frac{d k}{d T}\right\} \mathbf{z}
$$




$$
\begin{aligned}
\frac{d k}{d T} & =-\mathbf{e}+\mathbf{b} \times \frac{d R}{d T} \\
i \frac{d \mathbf{z}}{d T} & =\left\{\mathbf{M}_{\alpha} \cdot \mathbf{b}+\sum_{i}^{d} \mathcal{A}^{\alpha} \cdot \frac{d k}{d T}\right\} \mathbf{z} .
\end{aligned}
$$

$\mathbf{z}$ is a $\mathrm{CP}^{N-1}$ vector in FLs with $N$-fold degenerate conduction bands, i.e. $\mathbf{z}=\left(\mathrm{z}_{1}, \mathrm{z}_{2}, \cdots, \mathrm{z}_{N}\right)$ with $\mathbf{z}^{\dagger} \mathbf{z}=1$. This complex-valued vector describes the internal degrees of freedom associated with the degeneracy at each $k$-point. Correspondingly, the dual magnetic field, magnetic gauge field $\mathcal{A}^{\alpha}$ and "orbital magnetization" $\mathbf{M}_{\alpha}$ are all $N$ by $N$ Hermitian matrices. In a non-interacting fermi gas, they are defined solely in terms of the periodic part of Bloch wavefunctions of conduction bands $\left|u_{\sigma}\right\rangle(\sigma=1, \cdots, N \in \alpha)$;

$$
\begin{aligned}
\mathcal{B}_{i}^{\alpha} & \equiv i \epsilon_{i j m} \partial_{k_{j}} \mathcal{A}_{m}^{\alpha}+i \epsilon_{i j m} \mathcal{A}_{j}^{\alpha} \mathcal{A}_{m}^{\alpha} \\
{\left[\mathcal{A}_{j}^{\alpha}\right]_{\left(\sigma \mid \sigma^{\prime}\right)} } & \equiv\left\langle u^{\alpha \sigma} \mid \partial_{k_{j}} u^{\alpha \sigma^{\prime}}\right\rangle \\
{\left[\mathbf{M}_{\alpha, m}\right]_{\left(\sigma \mid \sigma^{\prime}\right)} } & \equiv \frac{i \epsilon_{m n l}}{2}\left\langle\partial_{k_{n}} u^{\alpha \sigma}\left|\hat{H}-E_{\alpha}\right| \partial_{k_{l}} u^{\alpha \sigma^{\prime}}\right\rangle
\end{aligned}
$$

with $E_{\alpha}$ being the energy dispersion for the $\alpha$-th band. Especially, the third EOM dictates that the $C P^{N-1}$ vector $\mathbf{z}$ precesses due to the nontrivial matrix structures of $\left[\mathbf{M}_{\alpha}\right]$ (Zeeman field) and $\left[\mathcal{A}_{j}^{\alpha}\right]$ (Wilczek-Zee phase) respectively.

One purpose of this paper is to enlarge the regime of validity of this effective EOM, so as to include metals for which electron-electron interactions are significant, i.e. Fermi liquids rather than Fermi gases. The $S U(N)$ effective EOM mentioned above is clearly valid only for a non-interacting Fermi gas, or within a mean-field description of ordered states such as (ferro)magnetic metals in which the ordered moment does not fluctuate at all. The fundamental framework of Fermi liquid theory, however, implies that these effective EOMs should be properly generalized into a realistic metal, where the electronelectron interaction/magnetic fluctuations are not weak. In fact, Haldane has recently argued that the "renormalized" Bloch wavefunction for a quasi-particle should be taken as the eigenvector of spectral function $\underline{21}, 23$ In this article, we provide a quite general derivation of the effective EOM for quasi-particles based on the Keldysh formalism, which confirms in part that this notion is valid in an arbitrary $U(1)$ Fermi liquid.

For $N=1$ case, the form of this EOM is identical to Eq.(11). However, in passing from the non-interacting version of gauge field definition, i.e. Eq.(3), to that of the many-body case, we encounter an additional complication. Because of the energy dependence of the self-energy, the renormalized Bloch wavefunction for quasi-particles - defined from the Green's function - depends on the energy or frequency $\omega$, in addition to crystal momentum $k$, i.e. $\left|u^{\alpha}(k, \omega)\right\rangle$. Accordingly, we are naturally led to introduce a sort of dual version of the electric field and associated electrostatic potential:

$$
\mathcal{E}_{j}^{\alpha} \equiv i\left(\partial_{\omega} \mathcal{A}_{j}^{\alpha}-\partial_{k_{j}} \mathcal{A}_{0}^{\alpha}\right)+i\left[\mathcal{A}_{0}^{\alpha}, \mathcal{A}_{j}^{\alpha}\right]
$$

$$
\left[\mathcal{A}_{0}^{\alpha}\right]_{\left(\sigma \mid \sigma^{\prime}\right)} \equiv\left\langle u^{\alpha \sigma} \mid \partial_{\omega} u^{\alpha \sigma^{\prime}}\right\rangle
$$

which, as well as the magnetic component, generally have non-trivial structures in the $(d+1)$-dimensional dual space.

As will be shown in this paper, the renormalized $k$ space Lorentz force appearing in the effective EOM for quasi-particles is composed both of these magnetic and electric component estimated on shell:

$$
\begin{aligned}
\tilde{\mathcal{B}}^{\alpha} & \equiv \overline{\mathcal{B}}^{\alpha}-\overline{\mathcal{E}}^{\alpha} \times \mathbf{v}_{\alpha}, \\
\overline{\mathcal{B}}^{\alpha} & \equiv\left(\mathcal{B}^{\alpha}\right)_{\mid \omega=\epsilon_{\alpha, k}}, \overline{\mathcal{E}}^{\alpha} \equiv\left(\mathcal{E}^{\alpha}\right)_{\mid \omega=\epsilon_{\alpha, k}}, \mathbf{v}_{\alpha, j}=\frac{\partial \epsilon_{\alpha, k}}{\partial k_{j}}(8)
\end{aligned}
$$

$\epsilon_{\alpha, k}$ is renormalized energy dispersion for a quasi-particle in question, which is defined as the pole of the singlepoint Green function with respect to $\omega$ (see Eq.(23)). In this sense, this newly introduced electric component provides another source of the Lorentz force in the $k$ space, particular only to interacting Fermi systems.

From the viewpoint of this EOM alone, however, the significance of the temporal component of the Berry's curvature is not clear. Namely, the renormalized Lorentz field $\tilde{\mathcal{B}}^{\alpha}$ can be also viewed as just the "magnetic component" defined in the codimensional space associated with the quasi-particle energy dispersion $\omega=\epsilon_{\alpha, k}$;

$$
\begin{aligned}
\tilde{\mathcal{B}}_{j}^{\alpha} & \equiv i \epsilon_{j m l} \partial_{k_{m}} \tilde{\mathcal{A}}_{k_{l}}^{\alpha}+i \epsilon_{j m l} \tilde{\mathcal{A}}_{k_{m}}^{\alpha} \tilde{\mathcal{A}}_{k_{l}}^{\alpha}, \\
\tilde{\mathcal{A}}_{k_{m}}^{\alpha} & \equiv\left\langle\tilde{u}^{\alpha} \mid \partial_{k_{m}} \tilde{u}^{\alpha}\right\rangle, \\
\left|\tilde{u}^{\alpha}(k)\right\rangle & \equiv\left|u^{\alpha}(k, \omega)\right\rangle_{\mid \omega=\epsilon_{\alpha, k}},
\end{aligned}
$$

(see Fig:11). Therefore, it is tempting to regard that the separate definition of the electric and magnetic fields, i.e. eqs. (215), is a sort of redundancy. Physically, this is somewhat sensible, since "sharply-peaked" quasiparticles ("infinite" life time with a definite dispersion relation $\left.\omega=\epsilon_{\alpha, k}\right)$ should be linked with those wavefunctions which are defined only in this codimensional subspace of the $\omega-k$ Euclidean space. Such thinking suggests that only derivatives along this subspace are meaningful, which thereby involve appropriate linear combinations of $\omega$ and $k$ derivatives.

Nevertheless, it can be shown that the dual electric and magnetic fields defined in the $\omega$ - $k$ Euclidean space do have distinct physical meaning. Specifically, we will show that these two fields enter into the linear response of the spectral weight to the applied electro and magnetic fields respectively (see eqs. (84-88) 24. This conclusion is obtained by solving the quasi-particle spectral function perturbatively with respect to the gradient expansion. Therein, we observe, in both $U(1)$ and $S U(2)$ case, that these Berry's curvatures characterize the 1st order correction to the weight of the quasi-particle spectral function. Since the spectral weight in principle can be detected in a momentum resolved way, one may at least envision several photoemission experiments as candidate tools to make a "contour map" of the dual electro and magnetic fields separately (see sec. IV). 


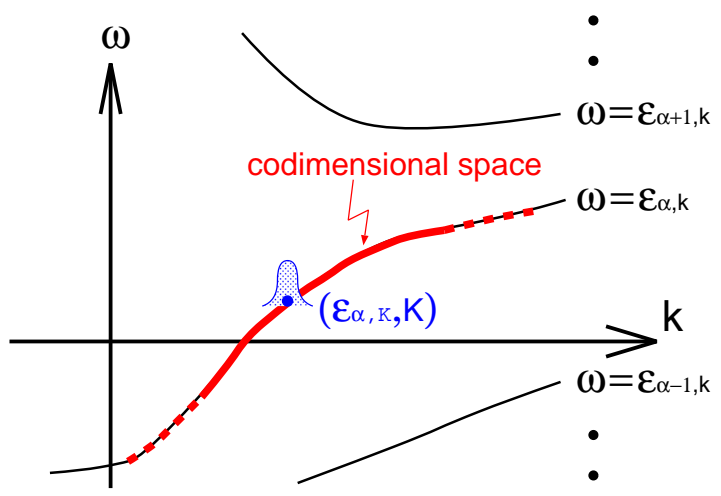

FIG. 1: $\omega-k$ Euclidean space and its codimensional subspace associated with $\omega=\epsilon_{\alpha, k}$ (Red line). $\tilde{\mathcal{B}}^{\alpha}$ can be viewed as just the "magnetic component" of Berry's curvature defined in latter subspace.

A momentum-resolved measurement of the dual electromagnetic fields is of interest not only as a matter of principle, but also potentially in diverse experimentally active areas such as anomalous and spin Hall effects in metals and semiconductors, and unconventional superconductivity in some ferromagnetic metals. Recent experimental activities in metals $13,15,16$ and semiconductors 11,12 have focused on novel observations of anomalous and spin Hall effects, leading to controversy over the origin of these effects. On the one hand are a variety of proposals of intrinsic effects, related to Berry phases and the above-defined dual electromagnetic fields in the clean band theory limit $\stackrel{6,12}{ }$ However, such Hall effects could also be of an extrinsic origin, i.e. a result of spin-dependent scattering of electrons from the spinorbit potential of impurity atoms 17 . Currently, the discrimination between these scenarios is rather indirect and based primarily upon the comparison to diverse theoretical model calculations and approximations whose physical applicability is difficult to judge $\underline{18}$. If one could somehow experimentally "visualize" the distribution of these dual electromagnetic fields in a momentum space, independently from Hall/galvanomagnetic measurements, experimentalists could readily judge for themselves whether intrinsic or extrinsic contributions are dominant in their material sample, by comparing with their transport measurements.

A second potential use for a measurement of dual electromagnetic fields stems for a recent proposal by Shi and $\mathrm{Niu}^{20}$ of a new formulation of the many-body problem based on the non-commutative quantum mechanics. They begin with a quantization of the EOM given in Eq.(11), leading to a non-zero commutator between position operators, $\left[\hat{R}_{j}, \hat{R}_{m}\right]=i \epsilon_{j m l} \mathcal{B}_{l}(\hat{k})^{\underline{6}}$, in addition to the usual commutators of momenta and position, i.e. $\left[\hat{k}_{j}, \hat{k}_{m}\right]=i \epsilon_{j m l} \mathbf{b}_{l}(\hat{R})$ and $\left[\hat{R}_{j}, \hat{k}_{m}\right]=i \delta_{j m}$. As is the case with the momentum in the presence of a real magnetic field $\mathbf{b}$, one can also introduce the canonical position op- erator $\hat{R}_{j}^{\prime} \equiv \hat{R}_{j}-i \mathcal{A}_{j}(\hat{k})$ such that their commutators become free from the dual magnetic field; $\left[\hat{R}_{j}^{\prime}, \hat{R}_{m}^{\prime}\right]=0$. At a price for this, however, the electron-electron interaction acquires an additional phase factor, which can transform a repulsive electron-electron interaction into an attractive one. Observing this, Shi and Niu attributed to the $k$-space Berry phase an unique origin of the superconducting phases realized only within ferromagnetic metals, such as in $\mathrm{UGe}_{2}, \mathrm{ZrZn}_{2}$ and URhGe. A detailed experimental information constraining $\mathcal{B}(k)$ as a function of $k$, in combination with a measurement of band dispersions would clearly provide these effective theoretical approaches an "ab-initio" model Hamiltonian, only to aid a quantitative comparison with material physics.

The structure of this paper is as follows. In section II, we introduce a quite general multiple-band continuum model and the Keldysh equation for the lesser (greater) Green function/spectral function. In section III, based on this (dissipationless) Keldysh equation, we provide a systematic procedure for carrying out the projection into the low-energy band, so as to obtain the effective (reduced) Keldysh equation for those Green functions of quasi-particles forming a Fermi surface.

Thanks to the Fermi liquid assumption, this effective Keldysh equation can be further solved in favor of the spectral function, perturbatively in the gradient expansion. Then, we observe in section IV that the Berry's curvatures, i.e. both dual electric fields and magnetic fields, indeed enter into the 1st order correction to the renormalization factor for quasi-particles. Using this solution for the spectral function, we further derive in section $\mathrm{V}$ the effective Boltzmann equation for the occupation number of quasi-particles in the phase space. Based on this equation, we can finally read off the $U(1)$ effective EOM in interacting Fermi liquids. Section VI is devoted to the summary and discussions of the present paper.

A number of appendices describe other topics useful in understanding the main text in more detail. For completeness, we briefly review the Keldysh formalism and our notations in the appendix A. The appendix B is devoted to demonstrating the logical consistency between our derived $U(1)$ effective EOM and the so-called Ishikawa-Matsuyawa-Haldane formula ${ }^{31}$ within the linear response regime. Appendix $\mathrm{C}$ describes how a $U(1)$ magnetic monopole "current" in $\omega-k$ space (which extends the notion of magnetic monopole density in $k$-space into the $\omega-k$ space) determines the distribution of dual electromagnetic fields in the $U(1)$ case. As a specific example which gives a quantitative idea of the significance of the many-body correction i.e. $\mathcal{E}$, we also present in appendix D some specific model calculations based on an electron-phonon coupling Hamiltonian. 


\section{KELDYSH FORMALISM}

\section{A. effective continuum model}

We begin with a general semi-microscopic model in which the electronic spectrum is described by a $k \cdot p$ type expansion about some (arbitrary) point in the Brillouin zone. One may keep as many bands as are deemed close enough in energy to be relevant to the physics, and our arguments do not depend upon the order in the expansion in $k$. Familiar examples would be the multiple-band Luttinger models, Dresselhaus models, and Rashba model commonly studied in semiconductors, in which the expansion point is chosen at the $\Gamma$ point. The advantage of this formulation is that we can Fourier transform, in a usual way, to a continuous real space coordinate $r$, i.e. $k \rightarrow-i \nabla_{r}$. The non-interacting Hamiltonian is thereby expressed in terms of the slowly varying "envelope fields" $\psi_{\alpha}(r)$ :

$$
\mathcal{H}_{0}=\sum_{\alpha, \alpha^{\prime}} \int d r \psi_{\alpha}^{\dagger}(r)\left[\hat{H}_{0}\left(-i \nabla_{r}, r, t\right)\right]_{\alpha \alpha^{\prime}} \psi_{\alpha^{\prime}}(r),
$$

where the band index $\alpha^{(\prime)}$ runs from 1 to $N_{b}$. Specific informations about lattice, orbital and spin-orbit couplings are encoded into the matrix structure of $\left[\hat{H}_{0}\right]$. Following the standard literatures ${ }^{25}$, we will employ a short-ranged electron-electron interaction potential;

$$
\begin{aligned}
\mathcal{H}_{1}=\sum \int & \int V_{\alpha_{1} \alpha_{2} \alpha_{2}^{\prime} \alpha_{1}^{\prime}}\left(r_{1}, r_{2}\right) \\
& \times \psi_{\alpha_{1}}^{\dagger}\left(r_{1}\right) \psi_{\alpha_{2}}^{\dagger}\left(r_{2}\right) \psi_{\alpha_{2}^{\prime}}\left(r_{2}\right) \psi_{\alpha_{1}^{\prime}}\left(r_{1}\right),
\end{aligned}
$$

though the form of our results does not depend in detail upon this.

\section{B. dissipationless Keldysh equation}

Apart from the perturbation theory at equilibrium, the Keldysh formalism is constituted by the lesser and greater Green functions;

$$
\begin{aligned}
& g^{>}\left(1 \mid 1^{\prime}\right) \equiv-i\left\langle\psi(1) \psi^{\dagger}\left(1^{\prime}\right)\right\rangle, \\
& g^{<}\left(1 \mid 1^{\prime}\right) \equiv i\left\langle\psi^{\dagger}\left(1^{\prime}\right) \psi(1)\right\rangle,
\end{aligned}
$$

where the time evolution of $\psi(1) \equiv \psi_{\alpha_{1}}\left(r_{1}, t_{1}\right)$ is determined by the interacting Hamiltonian introduced above;

$$
-i \frac{\partial \psi(1)}{\partial t_{1}} \equiv\left[\mathcal{H}_{0}+\mathcal{H}_{1}, \psi(1)\right]
$$

Because these Green functions are not the time-ordered ones, they acquire the translational symmetry in space and time at equilibrium. Furthermore, they are always Hermite matrices with respect to space and time coordinates and band indices.
Putting its derivation aside (see the Appendix A), let us begin with the Keldysh equation for these lesser and greater Green functions;

$$
\begin{aligned}
& {\left[G_{0}^{-1}-\Sigma^{\mathrm{HF}}-\sigma, \mathrm{g}^{<(>)}\right]_{\otimes,-}-\left[\Sigma_{\mathrm{c}}^{<(>)}, \mathrm{b}\right]_{\otimes,-}} \\
& =\frac{1}{2}\left[\Sigma_{\mathrm{c}}^{>}, \mathrm{g}^{<}\right]_{\otimes,+}-\frac{1}{2}\left[\Sigma_{\mathrm{c}}^{<}, \mathrm{g}^{>}\right]_{\otimes,+} .
\end{aligned}
$$

The (anti-)commutator here is defined by the convolution with respect to time $t$, space $r$ and band index $\alpha$;

$$
\begin{aligned}
{[\hat{B}, \hat{C}]_{\otimes, \pm}\left(1,1^{\prime}\right) \equiv \int } & d \overline{1} \hat{B}(1 \mid \overline{1}) \cdot \hat{C}\left(\overline{1} \mid 1^{\prime}\right) \\
& \pm \int d \overline{1} \hat{C}(1 \mid \overline{1}) \cdot \hat{B}\left(\overline{1} \mid 1^{\prime}\right)
\end{aligned}
$$

with $\int d 1 \equiv \sum_{\alpha_{1}} \int d r_{1} d t_{1}$. We denoted the bare Green function as $\hat{G}_{0}$ which is composed of the quadratic part of the Hamiltonian;

$$
\begin{aligned}
& \hat{G}_{0}^{-1}\left(1 \mid 1^{\prime}\right) \equiv \\
& {\left[i \partial_{t_{1}} \hat{1}-\hat{H}_{0}\left(-i \nabla_{r_{1}}, r_{1}, t_{1}\right)\right]_{\alpha_{1} \alpha_{1}^{\prime}} \delta\left(t_{1}-t_{1}^{\prime}\right) \delta^{d}\left(r_{1}-r_{1}^{\prime}\right) .}
\end{aligned}
$$

" $d$ " above represents the spatial dimension of our system.

Electron-electron interaction effects, on the other hand, are encoded in the self-energy such as $\hat{\Sigma}^{\mathrm{HF}}\left(1 \mid 1^{\prime}\right)$ and $\hat{\Sigma}_{\mathrm{c}}^{<(>)}\left(1 \mid 1^{\prime}\right)$, which are self-consistently given in terms of lesser/greater Green functions (see eqs. A5. A7) in the Appendix A). The former self-energy is the Hartree-Fock part, which is temporally instantaneous, i.e. $\quad \Sigma^{\mathrm{HF}}\left(1 \mid 1^{\prime}\right) \sim \delta\left(t_{1}-t_{1^{\prime}}\right)$. The latter one is usually dubbed as the lesser (greater) collisional self-energy, which is at least 2nd order in electron-electron interactions and thus temporally non-instantaneous. The physical role of this collisional self-energy is two-folded, corresponding to its following decomposition;

$$
\begin{aligned}
\sigma\left(1 \mid 1^{\prime}\right) & \equiv \frac{1}{2} \frac{t_{1}-t_{1^{\prime}}}{\left|t_{1}-t_{1^{\prime}}\right|}\left(\Sigma_{\mathrm{c}}^{>}\left(1 \mid 1^{\prime}\right)-\Sigma_{\mathrm{c}}^{<}\left(1 \mid 1^{\prime}\right)\right) \\
& \equiv \frac{1}{2}\left(\Sigma^{\mathrm{R}}\left(1 \mid 1^{\prime}\right)+\Sigma^{\mathrm{A}}\left(1 \mid 1^{\prime}\right)\right) \\
\Gamma\left(1 \mid 1^{\prime}\right) & \equiv i\left(\Sigma_{\mathrm{c}}^{>}\left(1 \mid 1^{\prime}\right)-\Sigma_{\mathrm{c}}^{<}\left(1 \mid 1^{\prime}\right)\right) \\
& \equiv i\left(\Sigma^{\mathrm{R}}\left(1 \mid 1^{\prime}\right)-\Sigma^{\mathrm{A}}\left(1 \mid 1^{\prime}\right)\right)
\end{aligned}
$$

with $\sigma\left(1 \mid 1^{\prime}\right)^{*} \equiv \sigma\left(1^{\prime} \mid 1\right)$ and $\Gamma\left(1 \mid 1^{\prime}\right)^{*} \equiv \Gamma\left(1^{\prime} \mid 1\right) . \Sigma^{\mathrm{R}}\left(1 \mid 1^{\prime}\right)$ and $\Sigma^{\mathrm{A}}\left(1 \mid 1^{\prime}\right)$ in the right hand side denotes the retarded and advanced self-energy respectively. Thus, $\sigma\left(1 \mid 1^{\prime}\right)$ stands for the real (Hermitian) part of the self-energy associated with the time-ordered Green function, which plays role of the renormalization of the quasi-particle energy and wavefunction. On the other hand, $\Gamma\left(1 \mid 1^{\prime}\right)$ is its imaginary (anti-hermitian) part, bringing about a finite life-time for quasi-particles.

In a same way, we can define the real/imaginary part of the Green function;

$$
\mathrm{b}\left(1 \mid 1^{\prime}\right) \equiv \frac{1}{2} \frac{t_{1}-t_{1^{\prime}}}{\left|t_{1}-t_{1^{\prime}}\right|}\left(\mathrm{g}^{>}\left(1 \mid 1^{\prime}\right)-\mathrm{g}^{<}\left(1 \mid 1^{\prime}\right)\right)
$$




$$
\begin{aligned}
& \equiv \frac{1}{2}\left(\mathrm{~g}^{\mathrm{R}}\left(1 \mid 1^{\prime}\right)+\mathrm{g}^{\mathrm{A}}\left(1 \mid 1^{\prime}\right)\right), \\
\mathrm{A}\left(1 \mid 1^{\prime}\right) & \equiv i\left(\mathrm{~g}^{>}\left(1 \mid 1^{\prime}\right)-\mathrm{g}^{<}\left(1 \mid 1^{\prime}\right)\right) \\
& \equiv i\left(\mathrm{~g}^{\mathrm{R}}\left(1 \mid 1^{\prime}\right)-\mathrm{g}^{\mathrm{A}}\left(1 \mid 1^{\prime}\right)\right),
\end{aligned}
$$

with $\mathrm{b}\left(1 \mid 1^{\prime}\right)^{*} \equiv \mathrm{b}\left(1^{\prime} \mid 1\right)$ and $\mathrm{A}\left(1 \mid 1^{\prime}\right)^{*} \equiv \mathrm{A}\left(1^{\prime} \mid 1\right)$. Note that especially the latter one is nothing but the spectral function. As is clear from eq. (13), the Keldysh equation for this spectral function is composed only by the real/imaginary part of the self-energy and Green functions introduced above;

$$
\left[G_{0}^{-1}-\Sigma^{\mathrm{HF}}-\sigma, \mathrm{A}\right]_{\otimes,-}-[\Gamma, \mathrm{b}]_{\otimes,-}=0 .
$$

We will dub $G_{0}^{-1}\left(1 \mid 1^{\prime}\right)-\Sigma^{\mathrm{HF}}\left(1 \mid 1^{\prime}\right)-\sigma\left(1 \mid 1^{\prime}\right)$ as a "Lagrangian" $\mathrm{L}\left(1 \mid 1^{\prime}\right)$ in a sense that, when Fouriertransformed with respect to its relative coordinate, i.e. $1-1^{\prime}$, it reduces to $\omega$ minus a "renormalized" Hamiltonian for quasi-particles (q.p.). Namely, its eigenvalues specify the renormalized energy dispersions for q.p. as their zeros with respect to $\omega$, while their corresponding eigenvectors constitute an orthogonal set. Latter of which therefore can be regarded as (a periodic part of) the renormalized Bloch wavefunction. Accordingly, we are led to define the Berry curvatures and gauge connections in the dual space in terms of the unitary matrix diagonalizing this Lagrangian (see eqs. (39), (38) and (21) respectively). As is shown in this paper, our effective Boltzmann/Keldysh equation legally derived via the projection process claims that the Berry's curvatures thus introduced indeed govern the effective EOM for q.p. in interacting Fermi systems.

On the other hand, the commutator between the antihermitian part of the self-energy $\Gamma$ and the hermitian part of the Green function $\mathrm{b}$ introduces a finite life time of q.p., i.e. the broadening of the spectral functions. When it comes to the q.p. closed to a Fermi surface, however, the Fourier-transformed $\Gamma$ as a function of $\omega$ becomes as small as $O\left((\omega-\mu)^{2}, T^{2}\right)$. Namely, the 2nd expression of eq. (15) dictates that, when analytically continued from a Matsubara Green function, $\Gamma$ at equilibrium is composed of a delta function, which imposes the energy conservation on its internal lines. As a result, the momentum integral regions associated with the internal lines are restricted to be only near a Fermi surface at low temperature $\mathrm{e}^{26}$.

$\Gamma$ in those electron-boson coupled systems with bosons having a finite excitation energy gets even smaller than this power-low decay. When $|\omega-\mu|$ is much smaller than this excitation energy $\omega_{0}$, (the lowest order) perturbative calculations readily show that $\hat{\Gamma}$ vanishes exponentially, such as $e^{-|\omega-\mu| / \omega_{0}}$ or $e^{-T / \omega_{0}}$ (see Appendix D). Anyway, in both of these two cases, the broadening of the q.p. spectral function at sufficiently low $T$ is by far smaller than the thermal line-broadening of the spectral function $(\sim T)$, which validates the so-called adiabatic assumption of Fermi liquid theory.

Meanwhile, the hermitian part of the collisional selfenergy $\hat{\sigma}$ remains finite even on a Fermi surface $(\omega=\mu)$ at zero temperature $(T=0)$. Namely, eq. (14) indicates that, when analytically continued from a Matsubara Green function, $\hat{\sigma}$ at equilibrium is composed of the principal integral rather than the delta function and thus free from the energy conservation imposed on its internal lines (see the appendix D for some example). As such, apart from the life time part $\Gamma$, its momentum integrals region for the internal lines are not restricted near the Fermi surface, which even causes the ultra-violet cut-off dependence of $\hat{\sigma}$ at $T=0$.

Because of these two different features generic in $\hat{\Gamma}$ and $\hat{\sigma}$, we ignore in this paper the (intrinsic) lifetime effect $\hat{\Gamma}$, while fully take into account the renormalization effects due to $\hat{\sigma}$. Namely, instead of eq. (18), we begin with the following dissipationless Keldysh equation for spectral functions:

$$
[\hat{\mathrm{L}}, \hat{\mathrm{A}}]_{\otimes,-}=\hat{0} .
$$

Even this $S U\left(N_{b}\right)$ dissipationless Keldysh equation is still non-trivial, due to the presence of the band index, whose effect is the central issue of this paper. In the following, we will present a general method of projecting out irrelevant band degrees of freedom associated with fully occupied bands and fully empty bands, so as to derive perturbatively the reduced Keldysh (kinetic) equation only for the relevant bands constituting a Fermi surface.

\section{REDUCED KELDYSH EQUATION}

\section{A. gradient expansion}

We will derive these reduced Keldysh equations perturbatively with respect to the gradient expansion. The coupling constant of this expansion is a dimensionless quantity which measures how much a system is disequilibrated. To define this expansion accurately, notice first that the lesser and greater Green function at equilibrium acquire the translational invariance in space and time coordinates; $\mathrm{g}^{<(>)}\left(1 \mid 1^{\prime}\right)=\mathrm{g}_{\alpha_{1} \alpha_{1^{\prime}}}^{<(>)}\left(r_{1}-r_{1^{\prime}}, t_{1}-t_{1^{\prime}}\right)$. Being given in terms of these Green functions, the lesser/greater self-energy and Lagrangian also become translationally invariant at equilibrium; $\mathrm{L}\left(1 \mid 1^{\prime}\right)=\mathrm{L}_{\alpha_{1} \alpha_{1^{\prime}}}\left(r_{1}-r_{1^{\prime}}, t_{1}-\right.$ $\left.t_{1^{\prime}}\right)$. As such, when Fourier-transformed, the convolution encoded in the dissipationless Keldysh equation reduces a simple product only with respect to band index;

$$
\begin{aligned}
& {[\hat{\mathrm{L}}(q, \omega), \hat{\mathrm{A}}(q, \omega)]_{-}=\hat{0},} \\
& {\left[\begin{array}{c}
\mathrm{L}_{\alpha \beta} \\
\mathrm{A}_{\alpha \beta}
\end{array}\right](q, \omega) \equiv \int d r d t e^{-i q r+\omega t}\left[\begin{array}{c}
\mathrm{L}_{\alpha \beta} \\
\mathrm{A}_{\alpha \beta}
\end{array}\right](r, t) .}
\end{aligned}
$$

Accordingly, we have only to diagonalize the Lagrangian so that an arbitrary diagonal $\hat{A}$ in this eigenbasis satisfies eq. (19) at equilibrium. We will regard this trivial limit as the non-perturbed case and take into account disequilibrations as perturbations. 
When a system is disequilibrated, the lesser/greater Green functions generally depend on the center of mass coordinate in space and time, i.e. $R \equiv \frac{r_{1}+r_{1^{\prime}}}{2}$ and $T \equiv$ $\frac{t_{1}+t_{1^{\prime}}}{2}$

$$
\mathrm{g}^{<(>)}\left(1 \mid 1^{\prime}\right)=\mathrm{g}_{\alpha_{1} \alpha_{1^{\prime}}}^{<(>)}(r, t ; R, T) .
$$

However, as long as a system is not so far from its equilibrium case, their dependences on $R$ and $T$ are slowly varying in comparison with the lattice spacing and inverse of band width respectively. As such, we could quantify the "distance" from equilibrium by a dimensionless ratio between this slowly varying length (time) scale and a lattice spacing (inverse of the band width). To extract the latter length/time scale, we have only to Fourier-transform the relative coordinate, i.e. $r$ and $t$, so that the crystal momentum $q$ and energy (frequency) $\omega$ are introduced 34 ;

$$
\begin{aligned}
& \hat{\mathrm{g}}^{<}(\omega, q ; T, R)=-i \int d r d t e^{-i q r+i \omega t} \hat{\mathrm{g}}^{<}(r, t ; R, T), \\
& \hat{\mathrm{g}}^{>}(\omega, q ; T, R)=i \int d r d t e^{-i q r+i \omega t} \hat{\mathrm{g}}^{>}(r, t ; R, T) .
\end{aligned}
$$

Then, the derivatives of these Green functions with re- spect to $q$ and $\omega$ are quantities of the order of the Fermi length and inverse of a band width respectively. Thus, the (inner) products between the derivatives of these Green functions with respect to $Q \equiv(\omega, q)$ and those with respect to $X \equiv(T, R)$ can be regarded as a small dimensionless quantity, as far as a system is only weakly disequilibrated;

$$
\hat{\mathrm{g}}^{a} \cdot \hat{\mathrm{g}}^{b} \gg \partial_{X} \hat{\mathrm{g}}^{a} \cdot \partial_{Q} \hat{\mathrm{g}}^{b} \gg \partial_{X} \partial_{X^{\prime}} \hat{\mathrm{g}}^{a} \cdot \partial_{Q} \partial_{Q^{\prime}} \hat{\mathrm{g}}^{b}, \cdots
$$

The superscripts " $a$ " and " $b$ " specify the lesser or greater green functions. Since Lagrangian and spectral function are given in terms of these functions self-consistently, we can readily adopt the following relations also:

$$
\begin{aligned}
\hat{\mathrm{L}} \cdot \hat{\mathrm{A}} & \gg \partial_{X} \hat{\mathrm{L}} \cdot \partial_{Q} \hat{\mathrm{A}}, \partial_{Q} \hat{\mathrm{L}} \cdot \partial_{X} \hat{\mathrm{A}} \\
& \gg \partial_{X} \partial_{X^{\prime}} \hat{\mathrm{L}} \cdot \partial_{Q} \partial_{Q^{\prime}} \hat{A}, \cdots
\end{aligned}
$$

Observing this, we expand the convolution in the (dissipationless) Keldysh equation, in powers of $\partial_{Q} \partial_{X} \equiv$ $-\partial_{\omega} \partial_{T}+\partial_{q_{j}} \partial_{R_{j}}$

$$
-[\hat{\mathrm{L}}, \hat{\mathrm{A}}]_{-}=\frac{i}{2}\left[\partial_{X_{j}} \hat{\mathrm{L}}, \partial_{Q_{j}} \hat{\mathrm{A}}\right]_{+}-\frac{1}{8}\left(\left[\partial_{X_{j}} \partial_{X_{k}} \hat{\mathrm{L}}, \partial_{Q_{j}} \partial_{Q_{k}} \hat{\mathrm{A}}\right]_{-}-\left\{X_{k} \leftrightarrow Q_{k}\right\}\right)-\left\{X_{j} \leftrightarrow Q_{j}\right\}+\cdots
$$

While we kept up to the 2nd order, one could explicitly write down the higher than this, by using the following formula for the Moyal product ${ }^{27}$;

$$
\begin{aligned}
(A \otimes B)(Q ; X) \equiv & \int d\left(1-1^{\prime}\right) e^{i Q \cdot\left(1-1^{\prime}\right)} \\
& \times \int d \overline{1} A(1 \mid \overline{1}) B\left(\overline{1} \mid 1^{\prime}\right), \\
= & e^{i \frac{1}{2}\left(\partial_{X}^{A} \partial_{Q}^{B}-\partial_{Q}^{A} \partial_{X}^{B}\right)} A(Q ; X) B(Q ; X),
\end{aligned}
$$

where $\partial_{X_{i}}^{A} \partial_{Q_{j}}^{B} \partial_{Q_{m}}^{A} \partial_{X_{l}}^{B}(A B) \equiv\left(\partial_{X_{i}} \partial_{Q_{m}} A\right)\left(\partial_{Q_{j}} \partial_{X_{l}} B\right)$. Note that the $j$ and $k$-summation in eq.(20) run from 0 to $d$, which will be made implicit from now on. The (anti-)commutators in eq.(20) are taken only with respect to band indices, while the non-local correlation effect encoded into the space-time convolution of eq.(19) is now perturbatively taken into account via the gradient expansion.

\section{B. projection process}

Our projection process is nothing but to solve eq. (20), perturbatively in the gradient expansion, using the $s u b$ stitution method. To be more specific, we are looking for
$\hat{A}$ which satisfies this equation at a given order accuracy in the gradient expansion. To do this in a well-controlled fashion, we will first solve the off-diagonal elements of the spectral function in favor of its diagonal elements, by looking into the off-diagonal components of the matrixformed KE given in eq. (20). Substituting these solutions back into the diagonal components of the same KE, we therefore obtain sort of differential equations given only for the diagonal elements of the spectral function (sec.III B,C,D). Then, we will further determine appropriate form of these diagonal elements, such that these differential equations are satisfied (sec. IV).

As in standard perturbation theories, we begin with diagonalizing the zero-th order part. Introduce the unitary matrix which diagonalizes the Lagrangian $\hat{L}$ in the left hand side of eq. (20);

$$
\hat{L}_{d} \equiv \hat{U}^{\dagger} \hat{\mathbf{L}} \hat{U} .
$$

Then, a spectral function in this basis has only to be diagonal so as to satisfy eq.(20) at the zero-th order;

$$
\hat{A} \equiv \hat{U}^{\dagger} \hat{\mathrm{A}} \hat{U}=\left[\begin{array}{ccc}
A_{1} & & \mathbf{0} \\
& \ddots & \\
\mathbf{0} & & A_{N_{b}}
\end{array}\right] .
$$


We used "SansSerif" for the spectral/green function represented in the old basis, while "Roman" for those in the new basis.

While each diagonal elements can be arbitrary at this level, from the physical point of view, they should be delta functions;

$$
A_{\alpha} \equiv \delta\left(L_{d, \alpha}^{(0)}\right)=Z_{\alpha}^{(0)} \delta\left(\omega-\epsilon_{\alpha, q}^{(0)}\right) .
$$

Namely, $\epsilon_{\alpha, q}$ above denotes the (renormalized) energy dispersion for the $\alpha$-th band q.p., while $Z_{\alpha}$ stands for this q.p. spectral weight. The superscript (0) simply represents that they are quantities of the zero-th order in gradient expansion. When employing this form as the 0 -th order solution, we will actually be able to satisfy the Keldysh equation up to the 1st order in the gradient expansion (see eq. (74)). Thus, we can justify posteriori that eq.(23) is the appropriate 0 -th order solution, based on which its higher order correction can be built up (see also the arguments in section IV).

Under this unitary transformation, the usual derivative encoded in Eq. (20) is replaced by the "covariant" derivative;

$$
\hat{U}^{\dagger}\left(\partial_{X} \hat{\mathrm{B}}\right) \hat{U}=\left[\hat{D}_{X}, \hat{B}\right] \equiv \partial_{X} \hat{B}+\hat{\mathcal{A}}_{X} \hat{B}-\hat{B} \hat{\mathcal{A}}_{X} .
$$

with $\hat{B}=U^{\dagger} \cdot \hat{\mathrm{B}} \cdot U$ and $\hat{\mathcal{A}}_{X}=\hat{U}^{\dagger} \partial_{X} \hat{U}$. Namely, in terms of this derivative, our matrix-formed differential equation reads

$$
-\left[\hat{L}_{d}, \hat{A}\right]=\frac{i}{2}\left[\left[\hat{D}_{X_{j}}, \hat{L}_{d}\right],\left[\hat{D}_{Q_{j}} \hat{A}\right]\right]_{+}+\cdots \equiv \hat{F}(\hat{A}) .
$$

To find the spectral function satisfying Eq. (25) up to higher order in the gradient expansion, let us next look into the off-diagonal components of this covariant differential equation;

$$
-L_{d, \alpha} A_{\alpha \beta}+A_{\alpha \beta} L_{d, \beta}=F_{\alpha \beta}\left(\left\{A_{\gamma}\right\},\left\{A_{\gamma \eta}\right\}\right) .
$$

$F_{\alpha \beta}$ is a functional of a set of diagonal elements of the spectral function, i.e. $\left\{A_{\gamma} \equiv A_{\gamma \gamma}\right\}$, and a set of its offdiagonal elements $\left\{A_{\gamma \eta}\right\}(\gamma \neq \eta)$. Notice first that the right hand side of eq. (26) is at least 1st order in gradient expansion. On the one hand, its left hand side is basically proportional to a direct band gap between $\alpha$-th band and $\beta$-th band, which is finite even at equilibrium, i.e. $L_{d, \alpha} \neq L_{d, \beta}$. Thus the off-diagonal elements of the spectral function are of the order of $\mathcal{O}\left(\left|\partial_{X} \partial_{Q}\right|\right)$, while its diagonal elements remain finite even at equilibrium.

As such, we first solve these off-diagonal elements in favor of the diagonal elements of the spectral function, iteratively in gradient expansion. Specifically, to the 1st order's accuracy, we have only to replace the off-diagonal elements in the right hand side of eq. (26) by zero;

$$
A_{\alpha \beta}=F_{\alpha \beta}\left(\left\{A_{\gamma}\right\},\{0\}\right) \cdot\left(L_{d, \beta}-L_{d, \alpha}\right)^{-1} \equiv A_{\alpha \beta}^{(1)} .
$$

Using this, one could further obtain the solution of $A_{\alpha \beta}$ to the 2 nd order accuracy;

$$
A_{\alpha \beta}^{(2)}\left(\left\{A_{\gamma}\right\}\right)=F_{\alpha \beta}\left(\left\{A_{\gamma}\right\},\left\{A_{\gamma \eta}^{(1)}\right\}\right),
$$

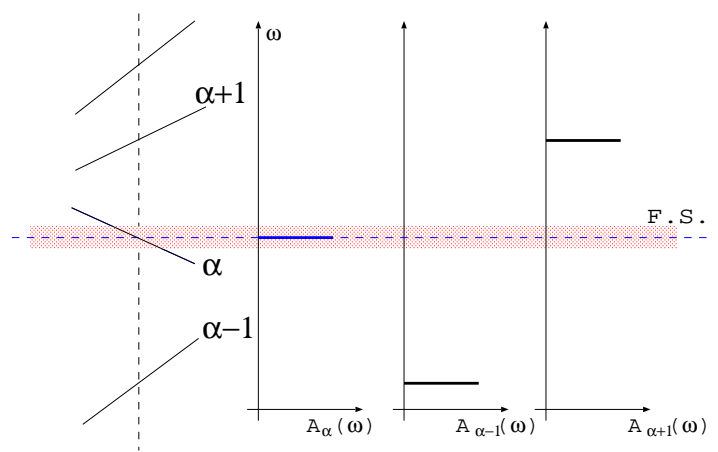

FIG. 2: A schematic picture of the spectral function for the $\alpha$-th band and its neighboring bands.

or higher than that,

$$
A_{\alpha \beta}^{(n+1)}\left(\left\{A_{\gamma}\right\}\right)=F_{\alpha \beta}\left(\left\{A_{\gamma}\right\},\left\{A_{\gamma \eta}^{(n)}\right\}\right) .
$$

When $n$ being infinity, this clearly becomes an exact relation between diagonal elements and off-diagonal elements.

Substituting these solutions into the diagonal components of the covariant differential equation, we then have $N_{b}$ decoupled equations which are given in terms only of a set of diagonal elements of the spectral function;

$$
\begin{aligned}
0 & =F_{\alpha}\left(\left\{A_{\gamma}\right\},\left\{A_{\gamma \eta}\right\}\right) \\
& =F_{\alpha}\left(\left\{A_{\gamma}\right\},\left\{A_{\gamma \eta} \equiv A_{\gamma \eta}^{(\infty)}\left(\left\{A_{\gamma}\right\}\right)\right\}\right),
\end{aligned}
$$

with $F_{\alpha} \equiv F_{\alpha \alpha}$. Then, a remaining task is to determine a set of $N_{b}$ diagonal elements of $\hat{A}$, such that they observe these equations. $\left\{A_{\gamma}\right\}$ thus obtained in combination with $A_{\gamma \eta} \equiv A_{\gamma \eta}^{(\infty)}\left(\left\{A_{\gamma}\right\}\right)$ are in principle equivalent to the exact solution of the an original dissipationless Keldysh equation, i.e. eq. (20).

However, the exact solution is apparently impossible, since it would require us, for example, to perform the iteration of eq. (29) at an infinite time. Thereby, we are going to indulge ourselves in executing this sequence of the process, up to a given order in gradient expansion. The highest order up to which we have succeeded in obtaining eq. (30) is currently the 2nd order. Up to this order, we can readily ignore the 3rd order gradient expansion term (and higher than that) denoted by "..." in eq. (20). Furthermore, $F_{\alpha \alpha}$ already containing at least one pair of $\partial_{X} \partial_{Q}$, the difference between eq. (27) and eq. (28) ends up with the 3rd order contributions, when substituted into $F_{\alpha \alpha}$ as in eq. (30). Thus, we are ready to use eq. (27).

The final simplification we will employ is that, for given $k, R$ and $T$, each quasi-particle bands have spectral weights at energetically well separated regions from one another. To be concrete, let us refer to a band which contains a Fermi surface as the $\alpha$-th band. Then, the diagonal elements of the spectral function corresponding to the other bands, i.e. $A_{\gamma \neq \alpha}$, have negligible weights at the 
low energy region, i.e. $\left|\omega-\epsilon_{\alpha}\right| \simeq|\omega-\mu| \ll \min _{\beta}\left|\Delta_{\alpha \beta}\right|$. This can be seen precisely at equilibrium, where $A_{\gamma \neq \alpha}$ is sharply peaked at an energy region separated from $\mu$ by the direct band gap $\Delta_{\gamma \alpha}$ (see Fig. 2). Even off equilibrium, higher order gradient expansion corrections to $A_{\gamma}$ turn out not to make additional incoherent weights other than the delta function we originally have at equilibrium. Namely, the corrections to $A_{\gamma}$ appear in eq. (23) only as the energy dispersion shift and the additive spectral weight (see for example eq. (88));

$$
\begin{gathered}
\epsilon_{\alpha}^{(0)} \rightarrow \epsilon_{\alpha}^{(0)}+\epsilon_{\alpha}^{(1)}, \\
Z_{\alpha}^{(0)} \rightarrow Z_{\alpha}^{(0)}+Z_{\alpha}^{(1)} .
\end{gathered}
$$

Accordingly, as far as this energy shift, i.e. $\epsilon_{\gamma}^{(1)}$, does not change the relative position of each quasi-particle energy dispersions, we are posterior $i$ allowed to replace $A_{\gamma \neq \alpha}$ by zero, for $\left|\omega-\epsilon_{\alpha}\right| \ll \min _{\beta} \Delta_{\alpha \beta}$.

To summarize the simplifications possible at the 2nd order analysis, we have only to derive the following equations;

$$
\begin{aligned}
0 & =F_{\alpha}\left(\left\{A_{\alpha}, A_{\gamma \neq \alpha} \equiv 0\right\},\left\{A_{\gamma \eta}^{(1)}\right\}\right), \\
A_{\gamma \eta}^{(1)} & \equiv F_{\gamma \eta}\left(\left\{A_{\alpha}, A_{\gamma \neq \alpha} \equiv 0\right\},\{0\}\right) \cdot\left(L_{d, \eta}-L_{d, \gamma}\right)^{-1} .
\end{aligned}
$$

In the next next subsection, we will substitute the latter into the former, so as to obtain the (differential) equation only for $A_{\alpha}$. We will dub the equation thus obtained as a reduced Keldysh equation (RKE). In the section IV, we will further find a $A_{\alpha}$ satisfying this reduced Keldysh equation. Thereby, we actually observe that $A_{\alpha}$ thus obtained is sharply peaked at $\omega=\epsilon_{\alpha}$, while having no incoherent weights at high energy sides. This observation will support posteriori the logical consistency built in our prescription described above.

\section{C. $S U(2)$ FLs and additional coupling constant}

In the argument of the previous subsection, we have implicitly assumed that a Fermi surface in question is composed only by a single band (especially eqs. (21/22)). In general, this is true either in those metals without any centrosymmetric lattice point or in ferromagnetic metals. In such FLs, there remains only a charge degree of freedom, while the (pseudo-)spin degree of freedom at each $k$-point is usually quenched. This charge degree of freedom is then described by the spectral function $A_{\alpha}$, which is a scalar quantity. Accordingly, the RKE, i.e. eq. (31), becomes just a differential equation for this scalar function, which we can name as a $U(1)$ RKE.

On the one hand, in usual paramagnetic metals having a centrosymmetric lattice point, each $k$-point is (at least) doubly degenerate, describing spin-degree of freedom for quasi-particles. Namely, eigenvalues of our Lagrangian are always two-folded at equilibrium. Thus, a Fermi surface is composed by two degenerate bands, which we could name as $S U(2) \mathrm{FL}$;

$$
\hat{L}_{d}=\hat{U}^{\dagger} \hat{\mathbf{L}} \hat{U}=\left[\begin{array}{ccc}
\hat{L}_{d, 1} & & \mathbf{0} \\
& \ddots & \\
\mathbf{0} & & \hat{L}_{d, N_{b}}
\end{array}\right] .
$$

Here 2 by 2 matrices $\hat{L}_{d, \gamma}$ should be proportional to a unit matrix at equilibrium.

As such, the spectral function which satisfies eq. (20) at the zero-th order in gradient expansion has only to be block-diagonalized,

$$
\hat{A}=\hat{U}^{\dagger} \hat{\mathrm{A}} \hat{U}=\left[\begin{array}{ccc}
\hat{A}_{1} & & \mathbf{0} \\
& \ddots & \\
\mathbf{0} & & \hat{A}_{N_{b}}
\end{array}\right],
$$

with arbitrary $2 \times 2$ matrices $\hat{A}_{\gamma}\left(\gamma=1, \cdots, N_{b}\right)$.

Corresponding to this generic degeneracy at the zeroth order, we will derive the RKEs in SU(2) FLs in favor of these 2 by 2 spectral functions, i.e. $\hat{A}_{\gamma}\left(\gamma=1, \cdots, N_{b}\right)$, so that spin and charge degrees of freedom are treated on an equal footing. As will be shown later, the RKE thus derived becomes a 2 by 2 matrix-formed differential equation, which we will dub as $S U(2)$ RKE.

Apart from relatively minor modifications, the derivation of the $S U(2)$ RKE also goes along with the same procedure as in $U(1)$ case. Specifically, it also begins with the inter-band components of $\mathrm{KE}$, which now take a 2 by 2 matrix-form;

$$
-\hat{L}_{d, \gamma} \hat{A}_{\gamma \eta}+\hat{A}_{\gamma \eta} \hat{L}_{d, \eta}=\hat{F}_{\gamma \eta}\left(\left\{\hat{A}_{\gamma}\right\},\left\{\hat{A}_{\gamma \eta}\right\}\right),
$$

where $\hat{A}_{\gamma \eta}$ is a 2 by 2 matrix, connecting the $\gamma$-th band and $\eta$-th band.

Being proportional to a unit matrix at equilibrium, 2 by 2 matrices $\hat{L}_{d, \gamma}$ may be decoupled into its zero-th order part and a small degeneracy lifting part;

$$
\hat{L}_{d, \gamma}=L_{d, \gamma}^{(0)} \hat{1}-\epsilon_{\gamma} \hat{\sigma}_{z} .
$$

A finite $\hat{\epsilon}_{\gamma} \equiv \epsilon_{\gamma} \hat{\sigma_{z}}$ is generally originated from weak $R$ and $T$-dependences of the Green functions. Thus $\epsilon_{\gamma}$ (divided by a characteristic band width) should be treated as a same order of quantity as $\left|\partial_{X} \partial_{Q}\right|$. To convince ourselves of this more directly, consider a specific situation in the presence of a small magnetic field $\mathbf{b}$, or equivalently a slowly varying magnetic gauge potential. In such a case, the Zeeman coupling energy between (bare) spin and $\mathbf{b}$ clearly should be included in $\hat{\epsilon}_{\gamma}$. On the one hand, $\left|\partial_{X} \partial_{Q}\right|$ contribution turns out to be proportional to a spatial derivative of the external gauge field, which is therefore proportional to $\mathbf{b}$ also (for example compare eq. (48) with eqs. (70-71)). As is obvious from this example, $\left|\partial_{X} \partial_{Q}\right| \equiv \lambda_{1}$ and $\epsilon / \Delta \equiv \lambda_{2}$ should be treated as same order of quantities;

$$
\lambda_{1} \sim \lambda_{2} \sim \lambda .
$$


Observing the inter-band components of the covariant differential equations, i.e. eq. (33), we first relate the inter-band elements of $\hat{A}$ with a set of $N_{b}$ intra-band elements of $\hat{A}$. To the 1 st order in $\lambda_{1}$ or $\lambda_{2}$, i.e. $\mathcal{O}\left(\lambda_{1}, \lambda_{2}\right)$, we have

$$
\hat{A}_{\gamma \eta}=\hat{F}_{\gamma \eta}\left(\left\{\hat{A}_{\gamma}\right\},\{\hat{0}\}\right) \cdot\left(\hat{L}_{d, \eta}-L_{d, \gamma}^{(0)} \hat{1}\right)^{-1} \equiv \hat{A}_{\gamma \eta}^{(1)} .
$$

Substituting these 1st order solutions into the intra-band components of $\mathrm{KE}$, we then obtain the following $S U(2)$ RKE to the accuracy of $\mathcal{O}\left(\lambda_{1}^{2}, \lambda_{2}^{2}, \lambda_{1} \lambda_{2}\right)$;

$$
\begin{aligned}
-\left[\hat{L}_{d, \alpha}, \hat{A}_{\alpha}\right] & =\left[\hat{\epsilon}_{\alpha}, \hat{A}_{\alpha}\right]=\hat{F}_{\alpha}\left(\left\{\hat{A}_{\alpha}, \hat{A}_{\gamma \neq \alpha} \equiv 0\right\},\left\{\hat{A}_{\gamma \eta}^{(1)}\right\}\right), \\
\hat{A}_{\gamma \eta}^{(1)}\left(\hat{A}_{\alpha}\right) & \equiv \hat{F}_{\gamma \eta}\left(\left\{\hat{A}_{\alpha}, \hat{A}_{\gamma \neq \alpha} \equiv 0\right\},\left\{\hat{A}_{\gamma \eta} \equiv \hat{0}\right\}\right) \cdot\left(\hat{L}_{d, \eta}-L_{d, \gamma}^{(0)} \hat{1}\right)^{-1} .
\end{aligned}
$$

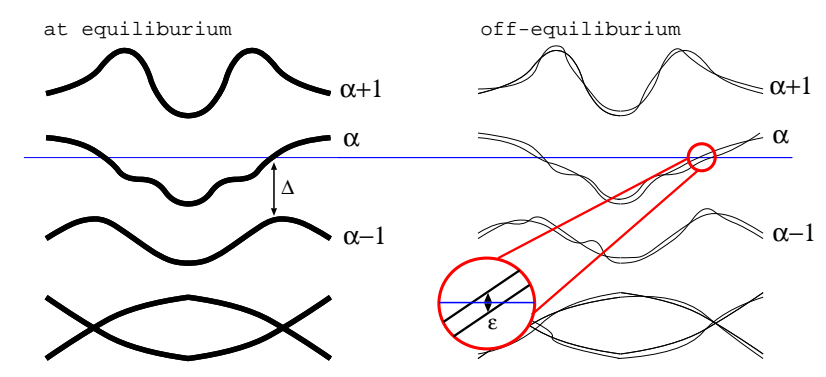

FIG. 3: A schematic picture of the energy dispersion in $S U(2)$ FLs. (Right): Each dispersion is doubly degenerate at equilibrium. (Left): When a system is weakly disequilibrated, the doubly degeneracy at each $k$-point is lifted. The associated splitting energy, i.e. $\epsilon$, is however much smaller than the typical band gap/width at equilibrium, i.e. $\Delta$.

The intra-band elements of $\hat{A}$ for the bands other than the $\alpha$-th band, i.e. $\hat{A}_{\gamma \neq \alpha}$, were already replaced by zero, because of the same reason as we argued in the $U(1)$ case.

In the next subsection, we will calculate eq. (35) in combination with eq. (36) more explicitly, so as to obtain an actual form of the $S U(2)$ RKE up to the order of $\mathcal{O}\left(\lambda^{2}\right)$. Out of the $S U(2)$ RKE thus derived, one can immediately obtain the RKE in $U(1) \mathrm{FLs}$, by regarding $\hat{A}_{\alpha}$ as a scalar function and putting $\hat{\epsilon}_{\alpha}$ to be zero.

\section{Actual Derivations of $S U(2)$ RKEs}

In this subsection, we will perform the actual calculation of eqs. (35) and (36) in a covariant way, only to arrive at the $S U(2)$ RKE associated with the $\alpha$-th band in question. By referring "in a covariant way", we mean that every step in the following manipulation does not change its explicit form either under an $S U(2)$ rotation $\hat{v}_{\alpha}$ within the $\alpha$-th band or, under an $S U\left(2 N_{b}-2\right)$ rotation $\hat{V}_{\bar{\alpha}}$ within its complementary space;

$$
\hat{U} \rightarrow \hat{U} \cdot\left[\begin{array}{cc}
\hat{v}_{\alpha} & \hat{0} \\
\hat{0} & \hat{V}_{\bar{\alpha}}
\end{array}\right] .
$$

This is because any steps of the actual calculation are composed either by the inter-band covariant derivatives or by the intra-band covariant derivatives, both of which will be accurately defined in the next subsubsection. In addition to their definitions, some formula frequently used in the next next subsubsection will be also summarized in advance for clarity.

\section{1. arithmetic preliminaries}

We have already defined the $S U\left(2 N_{b}\right)$ gauge field and associated "covariant" derivative in eq. (24). In an analogous way, the $S U(2)$ gauge fields and covariant derivatives for the two-fold degenerate $\alpha$-th band are defined as follows;

$$
\begin{gathered}
{\left[\hat{D}_{X}^{\alpha}, \hat{B}_{\alpha}\right] \equiv \partial_{X} \hat{B}_{\alpha}+\left[\hat{\mathcal{A}}_{X}^{\alpha}, \hat{B}_{\alpha}\right],} \\
{\left[\hat{\mathcal{A}}_{X}^{\alpha}\right]_{\left(\sigma \mid \sigma^{\prime}\right)} \equiv\left[\hat{U}^{\dagger} \partial_{X} \hat{U}\right]_{\left(\alpha \sigma \mid \alpha \sigma^{\prime}\right)},}
\end{gathered}
$$

where $X$ could be any coordinates in the phase space. We sub(super)scribe $\alpha$ or $\bar{\alpha}$ such as $\hat{B}_{\alpha}$ or $\hat{B}_{\bar{\alpha}}$, only to suggest that this matrix is a 2 by 2 matrix associated with the $\alpha$-th band or a $\left(2 N_{b}-2\right) \times\left(2 N_{b}-2\right)$ matrix in its complementary space respectively. When sub(super)scribed " $\alpha \bar{\alpha}$ " such as $\hat{B}_{\alpha \bar{\alpha}}^{(\alpha \bar{\alpha})}$, a matrix ought to be regarded as a $2 \times\left(2 N_{b}-2\right)$ matrix.

Apart from the derivative in the full Hilbert space, the covariant derivatives in its subspace do not commute with one another in general,

$$
\begin{aligned}
& {\left[\hat{D}_{X}^{\alpha},\left[\hat{D}_{X^{\prime}}^{\alpha}, \hat{B}_{\alpha}\right]\right]-\left[\hat{D}_{X^{\prime}}^{\alpha},\left[\hat{D}_{X}^{\alpha}, \hat{B}_{\alpha}\right]\right]=-i\left[\hat{\Omega}_{X X^{\prime}}^{\alpha}, \hat{B}_{\alpha}\right]} \\
& \hat{\Omega}_{X X^{\prime}}^{\alpha} \\
& \equiv i\left[\hat{D}_{X}^{\alpha}, \hat{D}_{X^{\prime}}^{\alpha}\right] \\
& \quad=i \partial_{X} \hat{\mathcal{A}}_{X^{\prime}}^{\alpha}-i \partial_{X^{\prime}} \hat{\mathcal{A}}_{X}^{\alpha}+i\left[\hat{\mathcal{A}}_{X}^{\alpha}, \hat{\mathcal{A}}_{X^{\prime}}^{\alpha}\right]
\end{aligned}
$$

As is clear from the latter expression, $\hat{\Omega}_{X X^{\prime}}^{\alpha}$ would be identical to zero, if this doubly degenerate $\alpha$-th band were to subtend a complete set, i.e. $\sum_{\sigma= \pm}\left|u^{\alpha \sigma}\right\rangle\left\langle u^{\alpha \sigma}\right| \equiv \hat{1}$.

Note that this derivative and the associated curvature $\hat{\Omega}_{X X^{\prime}}^{\alpha}$ transform in a covariant way under the unitary 
transformation in eq. (37);

$$
\begin{aligned}
\hat{B}_{\alpha} & \rightarrow \hat{v}_{\alpha}^{\dagger} \hat{B}_{\alpha} \hat{v}_{\alpha}, \\
{\left[\hat{D}_{X}^{\alpha}, \hat{B}_{\alpha}\right] } & \rightarrow \hat{v}_{\alpha}^{\dagger}\left[\hat{D}_{X}^{\alpha}, \hat{B}_{\alpha}\right] \hat{v}_{\alpha}, \\
\hat{\Omega}_{X X^{\prime}}^{\alpha} & \rightarrow \hat{v}_{\alpha}^{\dagger} \hat{\Omega}_{X X^{\prime}}^{\alpha} \hat{v}_{\alpha},
\end{aligned}
$$

while the gauge field $\hat{\mathcal{A}}_{X}^{\alpha}$ is not;

$$
\hat{\mathcal{A}}_{X}^{\alpha} \rightarrow \hat{v}_{\alpha}^{\dagger} \hat{\mathcal{A}}_{X}^{\alpha} \hat{v}_{\alpha}+\hat{v}_{\alpha}^{\dagger} \partial_{X} \hat{v}_{\alpha} .
$$

In addition to this gauge field in the $\alpha$-th band, we can also define the inter-band gauge fields as the $2 \times\left(2 N_{b}-2\right)$ off-diagonal blocks of $\hat{\mathcal{A}}_{X}$;

$$
\hat{\mathcal{A}}_{X} \equiv \hat{U}^{\dagger} \partial_{X} \hat{U} \equiv\left[\begin{array}{cc}
\hat{\mathcal{A}}_{X}^{\alpha} & \hat{\mathcal{A}}_{X}^{\alpha \bar{\alpha}} \\
\hat{\mathcal{A}}_{X}^{\bar{\alpha} \alpha} & \hat{\mathcal{A}}_{X}^{\bar{\alpha}}
\end{array}\right] .
$$

Under the unitary transformation defined in eq. (37), the inter-band gauge field clearly transforms in a "covariant" way;

$$
\hat{\mathcal{A}}_{X}^{\alpha \bar{\alpha}} \rightarrow \hat{v}_{\alpha}^{\dagger} \hat{\mathcal{A}}_{X}^{\alpha \bar{\alpha}} \hat{V}_{\bar{\alpha}},
$$

while the intra-band gauge fields such as $\hat{\mathcal{A}}_{X}^{\alpha}$ and $\hat{\mathcal{A}}_{X}^{\bar{\alpha}}$ are not.

Using the latter ones, we can further define a $2 \times\left(2 N_{b}-\right.$ 2) matrix-form derivative, which plays role of a sort of inter-band covariant derivative;

$$
\left[\hat{D}_{X}^{\alpha \bar{\alpha}}, \hat{B}_{\alpha \bar{\alpha}}\right] \equiv \partial_{X} \hat{B}_{\alpha \bar{\alpha}}+\hat{\mathcal{A}}_{X}^{\alpha} \hat{B}_{\alpha \bar{\alpha}}-\hat{B}_{\alpha \bar{\alpha}} \hat{\mathcal{A}}_{X}^{\bar{\alpha}}
$$

where $\hat{B}_{\alpha \bar{\alpha}}$ stands for an arbitrary $2 \times\left(2 N_{b}-2\right)$ matrix. As long as this matrix is a covariant quantity, i.e. $\hat{B}_{\alpha \bar{\alpha}} \rightarrow \hat{v}_{\alpha}^{\dagger} \hat{B}_{\alpha \bar{\alpha}} \hat{V}_{\bar{\alpha}}$, the inter-band derivative above clearly transforms in a covariant way under eq. (37);

$$
\left[\hat{D}_{X}^{\alpha \bar{\alpha}}, \hat{B}_{\alpha \bar{\alpha}}\right] \rightarrow \hat{v}_{\alpha}^{\dagger}\left[\hat{D}_{X}^{\alpha \bar{\alpha}}, \hat{B}_{\alpha \bar{\alpha}}\right] \hat{V}_{\bar{\alpha}} .
$$

In terms of these quantities and derivatives, let us summarize henceforth several formula which become useful in the next subsubsection. Consider first a inter-band covariant derivative of a inter-band gauge field;

$$
\begin{aligned}
& {\left[\hat{D}_{X}^{\alpha \bar{\alpha}}, \hat{\mathcal{A}}_{X^{\prime}}^{\alpha \bar{\alpha}}\right]_{\left(\sigma \mid \beta \sigma^{\prime}\right)}} \\
& =\left[\left(\partial_{X} \hat{U}^{\dagger}\right) \partial_{X^{\prime}} \hat{U}\right]_{\left(\alpha \sigma \mid \beta \sigma^{\prime}\right)}+\left[\hat{U}^{\dagger} \partial_{X X^{\prime}}^{2} \hat{U}\right]_{\left(\alpha \sigma \mid \beta \sigma^{\prime}\right)} \\
& \quad-\sum_{\sigma^{\prime \prime}= \pm}\left[\left(\partial_{X} \hat{U}^{\dagger}\right) \hat{U}\right]_{\left(\alpha \sigma \mid \alpha \sigma^{\prime \prime}\right)}\left[\hat{U}^{\dagger} \partial_{X^{\prime}} \hat{U}\right]_{\left(\alpha \sigma^{\prime \prime} \mid \beta \sigma^{\prime}\right)} \\
& \quad+\sum_{\gamma \neq \alpha} \sum_{\sigma^{\prime \prime}= \pm}\left[\left(\partial_{X^{\prime}} \hat{U}^{\dagger}\right) \hat{U}\right]_{\left(\alpha \sigma \mid \gamma \sigma^{\prime \prime}\right)}\left[\hat{U}^{\dagger} \partial_{X} \hat{U}\right]_{\left(\gamma \sigma^{\prime \prime} \mid \beta \sigma^{\prime}\right)},
\end{aligned}
$$

where $\beta \neq \alpha$ and $\sigma, \sigma^{\prime}= \pm$. Then, applying into the last two terms the following identity,

$$
\sum_{\sigma= \pm}|\alpha \sigma\rangle\left\langle\alpha \sigma\left|=\hat{1}-\sum_{\gamma \neq \alpha} \sum_{\sigma= \pm}\right| \gamma \sigma\right\rangle\langle\gamma \sigma|
$$

we can exchange the subscripts of the covariant derivative and the gauge field with each other;

$$
\left[\hat{D}_{X}^{\alpha \bar{\alpha}}, \hat{\mathcal{A}}_{X^{\prime}}^{\alpha \bar{\alpha}}\right]_{\left(\sigma \mid \beta \sigma^{\prime}\right)} \equiv\left[\hat{D}_{X^{\prime}}^{\alpha \bar{\alpha}}, \hat{\mathcal{A}}_{X}^{\alpha \bar{\alpha}}\right]_{\left(\sigma \mid \beta \sigma^{\prime}\right)} .
$$

In the actual calculations, this equality becomes very powerful, when combined with the "decomposition rule" of covariant derivatives such as;

$$
\begin{aligned}
& {\left[\hat{D}_{X}^{\alpha}, \hat{B}_{\alpha \bar{\alpha}} \hat{B}_{\bar{\alpha}}^{\prime} \hat{B}_{\bar{\alpha} \alpha}^{\prime \prime}\right]=\left[\hat{D}_{X}^{\alpha \bar{\alpha}}, \hat{B}_{\alpha \bar{\alpha}}\right] \hat{B}_{\bar{\alpha}}^{\prime} \hat{B}_{\bar{\alpha} \alpha}^{\prime \prime}} \\
& \quad+\hat{B}_{\alpha \bar{\alpha}}\left[\hat{D}_{X}^{\bar{\alpha}}, \hat{B}_{\bar{\alpha}}^{\prime}\right] \hat{B}_{\bar{\alpha} \alpha}^{\prime \prime}+\hat{B}_{\alpha \bar{\alpha}} \hat{B}_{\bar{\alpha}}^{\prime}\left[\hat{D}_{X}^{\bar{\alpha} \alpha}, \hat{B}_{\bar{\alpha} \alpha}^{\prime \prime}\right] .
\end{aligned}
$$

One should also note that curvatures either in the $\alpha$-th band space or in its complementary space can be expressed also in terms of inter-band gauge fields;

$$
\begin{aligned}
& \hat{\Omega}_{X X^{\prime}}^{\alpha}=-i\left(\hat{\mathcal{A}}_{X}^{\alpha \bar{\alpha}} \hat{\mathcal{A}}_{X^{\prime}}^{\bar{\alpha} \alpha}-\hat{\mathcal{A}}_{X^{\prime}}^{\alpha \bar{\alpha}} \hat{\mathcal{A}}_{X}^{\bar{\alpha} \alpha}\right), \\
& \hat{\Omega}_{X X^{\prime}}^{\bar{\alpha}}=-i\left(\hat{\mathcal{A}}_{X}^{\bar{\alpha} \alpha} \hat{\mathcal{A}}_{X^{\prime}}^{\alpha \bar{\alpha}}-\hat{\mathcal{A}}_{X^{\prime}}^{\bar{\alpha} \alpha} \hat{\mathcal{A}}_{X}^{\alpha \bar{\alpha}}\right) .
\end{aligned}
$$

\section{2. $S U(2) \times S U\left(2 N_{b}-2\right)$ covariant manipulations}

Using the arithmetics described so far, we will study eqs. (3556) within the 2nd order accuracy in $\lambda$. As is the case for a standard (such as RayleighSchrodinger) perturbation theory, our 2nd order expression for eqs. (35136) is given both by eigen-energies at the zero-th order and its eigen-wavefunctions (see eq. (55) for example). Namely, differences between eigenvalues of $\hat{L}$ enter into a sort of "energy-denominator", while a "numerator" in a usual perturbation theory is now transcribed into a gauge field (connection), which is nothing but the matrix element of our perturbation part (i.e. $\partial_{X} \partial_{Q}$ ) among different eigenbases of $\hat{L}$. Accordingly, just as in a usual perturbation theory, our 2nd order expression for eqs. (35136) also depends on wavefunctions and eigenvalues not only for the $\alpha$-th band in question, but also for the bands other than the $\alpha$-th band.

On the other hand, extensive semi-classical analyses in a non-interacting system $5,6,7,8,9,10$ suggest that the low-energy effective theory for (quasi-)particles should be constituted only by Bloch wavefunctions and energy dispersions for the $\alpha$-th band in question, while free from details of the other bands.

We shall show in this subsubsection that this is indeed the case for the $S U(2) \mathrm{RKE}$ given in eqs. (35|36) at least up to 2 nd order in $\lambda$. To be more specific, we will transform eq. (35) in combination with eq. (36) into a more compact form rigorously up to $\mathcal{O}\left(\lambda^{2}\right)$, only to find that they actually are given solely in terms of the $S U(2)$ gauge covariant quantities such as $\hat{\Omega}_{X X^{\prime}}^{\alpha}$ and $\left[\hat{D}_{X}^{\alpha}, \cdots\right]$. During this transformation, several formula described in the previous subsubsection, such as eqs. (42,45), become very useful.

To see this, let us begin with eq. (35), i.e. the $(\alpha, \alpha)$-th component (diagonal component) of the original dissipationless Keldysh equation;

$$
-\left[\hat{L}_{d}, \hat{A}_{\alpha}\right]=\hat{F}_{\alpha}^{(1)}\left(\hat{A}_{\alpha}\right)+F_{\alpha}^{(2)}\left(\left\{\hat{A}_{\alpha \eta}\right\}\right)+F_{\alpha}^{(3)}\left(\left\{\hat{A}_{\eta \delta}\right\}\right)
$$

For the later clarity, the right hand side was decoupled with respect to different elements of $\hat{A}$. This becomes possible clearly because our differential equation is at 
most linear in $\hat{A}$. Up to 1 st order in $\lambda_{1}$ the first term $\hat{L}_{d}$; in the right hand side reads; $\underline{28}$

$$
\begin{gathered}
\hat{F}_{\alpha}^{(1)}\left(\hat{A}_{\alpha}\right)=\frac{i}{2}\left[\left[\hat{D}_{X_{j}}^{\alpha}, \hat{L}_{d, \alpha}\right],\left[\hat{D}_{Q_{j}}^{\alpha}, \hat{A}_{\alpha}\right]\right]_{+}-\left\{X_{j} \leftrightarrow Q_{j}\right\} \\
-\left[\hat{\mathcal{M}}_{\alpha}, \hat{A}_{\alpha}\right]_{-}-\frac{1}{4}\left[\hat{\mathcal{N}}_{\alpha}, \hat{A}_{\alpha}\right]_{+}+\mathcal{O}\left(\lambda_{1}^{2}\right),
\end{gathered}
$$

where we have introduced following 2 by 2 hermite and anti-hermite matrices respectively;

$$
\begin{aligned}
\hat{\mathcal{M}}_{\alpha} & \equiv \frac{i}{2}\left\{\hat{\mathcal{A}}_{Q_{j}}^{\alpha \bar{\alpha}}\left(\hat{L}_{d, \bar{\alpha}}-L_{d, \alpha}^{(0)} \hat{1}\right) \hat{\mathcal{A}}_{X_{j}}^{\bar{\alpha} \alpha}-\left\{X_{j} \leftrightarrow Q_{j}\right\}\right\} \\
\hat{\mathcal{N}}_{\alpha} & \equiv\left[\hat{\epsilon}_{\alpha}, \hat{\Omega}_{X_{j} Q_{j}}^{\alpha}\right]_{-} .
\end{aligned}
$$

$\hat{L}_{d, \bar{\alpha}}$ denotes a $\left(2 N_{b}-2\right) \times\left(2 N_{b}-2\right)$ diagonal block of

Observing eq. (47), notice first the commutator between $\hat{\mathcal{M}}_{\alpha}$ and the $\alpha$-th band spectral function, i.e $\hat{A}_{\alpha}$. This commutator implies that the former hermitian matrix is the 1 st order correction to the $\alpha$-th band dispersion. Notice also that $\hat{\mathcal{N}}_{\alpha}$ enters into the anti-commutator with $\hat{A}_{\alpha}$. As will be shown later, this anti-commutator lets $\hat{\mathcal{N}}_{\alpha}$ play a relevant role in determining the 1st order gradient expansion correction to the spectral weight (see the section. IV for details).

The 2nd order contributions in $\hat{F}_{\alpha}^{(1)}\left(\hat{A}_{\alpha}\right)$ and $\hat{F}_{\alpha}^{(2)}\left(\hat{A}_{\alpha \bar{\alpha}}\right)$ and $\hat{F}_{\alpha}^{(3)}\left(\hat{A}_{\bar{\alpha}}\right)$ are given as follows;

$$
\begin{aligned}
\hat{F}^{(1)}\left(\hat{A}_{\alpha}\right)= & \cdots-\frac{1}{8}\left\{\partial_{X_{j}} \partial_{X_{k}} L_{d, \alpha}^{(0)} \hat{1}+\hat{\mathcal{A}}_{X_{j}}^{\alpha \bar{\alpha}}\left(L_{d, \alpha}^{(0)} \hat{1}-\hat{L}_{d, \bar{\alpha}}\right) \hat{\mathcal{A}}_{X_{k}}^{\bar{\alpha} \alpha}+\hat{\mathcal{A}}_{X_{k}}^{\alpha \bar{\alpha}}\left(L_{d, \alpha}^{(0)} \hat{1}-\hat{L}_{d, \bar{\alpha}}\right) \hat{\mathcal{A}}_{X_{j}}^{\bar{\alpha} \alpha}\right\} \\
& \cdot\left\{\left[D_{Q_{j}}^{\alpha},\left[D_{Q_{k}}^{\alpha}, \hat{A}_{\alpha}\right]\right]+\hat{\mathcal{A}}_{Q_{j}}^{\alpha \bar{\alpha}} \hat{\mathcal{A}}_{Q_{k}}^{\bar{\alpha} \alpha} \hat{A}_{\alpha}+\hat{A}_{\alpha} \hat{\mathcal{A}}_{Q_{k}}^{\alpha \bar{\alpha}} \hat{\mathcal{A}}_{Q_{j}}^{\bar{\alpha} \alpha}\right\} \\
- & \frac{1}{8}\left\{-\mathcal{A}_{X_{k}}^{\alpha \bar{\alpha}}\left[D_{X_{j}}^{\bar{\alpha}}, L_{d, \alpha}^{(0)} \hat{1}-\hat{L}_{d, \bar{\alpha}}\right]-\mathcal{A}_{X_{j}}^{\alpha \bar{\alpha}}\left[D_{X_{k}}^{\bar{\alpha}}, L_{d, \alpha}^{(0)} \hat{1}-\hat{L}_{d, \bar{\alpha}}\right]-\left[D_{X_{j}}^{\alpha \bar{\alpha}}, \hat{\mathcal{A}}_{X_{k}}^{\alpha \bar{\alpha}}\right]\left(L_{d, \alpha}^{(0)} \hat{1}-\hat{L}_{d, \bar{\alpha}}\right)\right\} \\
& \cdot\left\{\left[D_{Q_{j}}^{\bar{\alpha} \alpha}, \hat{\mathcal{A}}_{Q_{k}}^{\bar{\alpha} \alpha} \hat{A}_{\alpha}+\hat{\mathcal{A}}_{Q_{k}}^{\bar{\alpha} \alpha}\left[D_{Q_{j}}^{\alpha}, \hat{A}_{\alpha}\right]+\hat{\mathcal{A}}_{Q_{j}}^{\bar{\alpha} \alpha}\left[D_{Q_{k}}^{\alpha}, \hat{A}_{\alpha}\right]\right\}\right. \\
& -\left\{X_{j} \leftrightarrow Q_{j} \mid X_{k}, Q_{k}\right\}-\left\{X_{j}, Q_{j} \mid X_{k} \leftrightarrow Q_{k}\right\}+\left\{X_{j} \leftrightarrow Q_{j} \mid X_{k} \leftrightarrow Q_{k}\right\}-\text { h.c. }+\mathcal{O}\left(\lambda^{3}\right) . \\
\hat{F}_{\alpha}^{(2)}\left(\hat{A}_{\alpha \bar{\alpha}}\right)= & \frac{i}{2}\left\{\partial_{X_{j}} L_{d, \alpha}^{(0)}\left(\hat{\mathcal{A}}_{Q_{j}}^{\alpha \bar{\alpha}} \hat{A}_{\bar{\alpha} \alpha}-\hat{A}_{\alpha \bar{\alpha}} \hat{\mathcal{A}}_{Q_{j}}^{\bar{\alpha} \alpha}\right)-\hat{\mathcal{A}}_{X_{j}}^{\alpha \bar{\alpha}}\left(L_{d, \alpha}^{(0)} \hat{1}-\hat{L}_{d, \bar{\alpha}}\right)\left[D_{Q_{j}}^{\bar{\alpha} \alpha}, \hat{A}_{\bar{\alpha} \alpha}\right]\right\} \\
& -\left\{X_{j} \leftrightarrow Q_{j}\right\}-\text { h.c. }+\mathcal{O}\left(\lambda^{2}\right), \\
\hat{F}_{\alpha}^{(3)}\left(\hat{A}_{\bar{\alpha}}\right)= & \frac{i}{2} \hat{\mathcal{A}}_{Q_{j}}^{\alpha \bar{\alpha}} \hat{A}_{\bar{\alpha}}\left(L_{d, \alpha}^{(0)} \hat{1}-\hat{L}_{d, \bar{\alpha}}\right) \hat{\mathcal{A}}_{X_{j}}^{\bar{\alpha} \alpha}-\left\{X_{j} \leftrightarrow Q_{j}\right\}-\text { h.c. }+\mathcal{O}\left(\lambda^{2}\right) .
\end{aligned}
$$

where "..." in $\hat{F}^{(1)}$ stands for those terms explicit in the right hand side of eq. (47). To rewrite the latter two, i.e. $\hat{F}_{\alpha}^{(2)}$ and $\hat{F}_{\alpha}^{(3)}$, only in terms of $\hat{A}_{\alpha}$, we first solve $\hat{A}_{\alpha \bar{\alpha}}$ and $\hat{A}_{\bar{\alpha}}$ in favor of $\hat{A}_{\alpha}$;

$$
\begin{aligned}
\hat{A}_{\alpha \bar{\alpha}}\left(\hat{A}_{\alpha}\right)= & \frac{i}{2}\left\{-\hat{A}_{\alpha} \hat{\mathcal{A}}_{Q_{k}}^{\alpha \bar{\alpha}}\left(\partial_{X_{k}} L_{d, \alpha}^{(0)} \hat{1}+\left[D_{X_{k}}^{\bar{\alpha}}, \hat{L}_{d, \bar{\alpha}}\right]\right)+\left[D_{Q_{k}}^{\alpha}, \hat{A}_{\alpha}\right] \hat{\mathcal{A}}_{X_{k}}^{\alpha \bar{\alpha}}\left(\hat{L}_{d, \bar{\alpha}}-L_{d, \alpha}^{(0)} \hat{1}\right)\right\} \cdot\left(\hat{L}_{d, \bar{\alpha}}-L_{d, \alpha}^{(0)} \hat{1}\right)^{-1} \\
& -\left\{X_{k} \leftrightarrow Q_{k}\right\}+\mathcal{O}\left(\lambda^{2}\right), \\
\hat{A}_{\eta \delta}\left(\hat{A}_{\alpha}\right)= & \frac{i}{2}\left(\hat{L}_{d, \eta} \hat{\mathcal{A}}_{X_{k}}^{\eta \alpha} \hat{A}_{\alpha} \hat{\mathcal{A}}_{Q_{k}}^{\alpha \delta}-\hat{\mathcal{A}}_{X_{k}}^{\eta \alpha} \hat{A}_{\alpha} \hat{\mathcal{A}}_{Q_{k} \delta}^{\alpha \delta} \hat{L}_{d, \delta}\right) \cdot\left(\hat{L}_{d, \delta}-\hat{L}_{d, \eta}\right)^{-1}-\left\{X_{k} \leftrightarrow Q_{k}\right\}+\mathcal{O}\left(\lambda^{2}\right), \\
= & -\frac{i}{2} \hat{\mathcal{A}}_{X_{k}}^{\eta \alpha} \hat{A}_{\alpha} \hat{\mathcal{A}}_{Q_{k}}^{\alpha \delta}-\left\{X_{k} \leftrightarrow Q_{k}\right\}+\mathcal{O}\left(\lambda^{2}\right),
\end{aligned}
$$

with $\eta, \delta \neq \alpha$. One can obtain these relations, by looking into the inter-band components of the dissipationless Keldysh equation, precisely as in eq.(36). We then substitute these two back into eqs. (5152), only to obtain $\hat{F}_{\alpha}^{(2)}$ and $\hat{F}_{\alpha}^{(3)}$ as a functional of $\hat{A}_{\alpha}$;

$$
\begin{aligned}
\hat{F}_{\alpha}^{(2)}\left(\hat{A}_{\alpha}\right)= & \frac{1}{2}\left(\partial_{X_{j}} L_{d, \alpha}^{(0)}\right) \hat{A}_{\alpha} \hat{\mathcal{A}}_{Q_{k}}^{\alpha \bar{\alpha}}\left[\hat{D}_{X_{k}}^{\bar{\alpha}}, L_{d, \alpha}^{(0)} \hat{1}+\hat{L}_{d, \bar{\alpha}}\right]\left[L_{d, \alpha}^{(0)} \hat{1}-\hat{L}_{d, \bar{\alpha}}\right]^{-1} \hat{\mathcal{A}}_{Q_{j}}^{\bar{\alpha} \alpha}+\frac{1}{2}\left(\partial_{X_{j}} L_{d, \alpha}^{(0)}\right)\left[\hat{D}_{Q_{k}}^{\alpha}, \hat{A}_{\alpha}\right] \hat{\mathcal{A}}_{X_{k}}^{\alpha \bar{\alpha}} \hat{\mathcal{A}}_{Q_{j}}^{\bar{\alpha} \alpha} \\
& -\frac{1}{4}\left[\hat{D}_{Q_{j}}^{\alpha}, \hat{A}_{\alpha}\right] \hat{\mathcal{A}}_{Q_{k}}^{\alpha \bar{\alpha}}\left[\hat{D}_{X_{k}}^{\bar{\alpha}}, L_{d, \alpha}^{(0)} \hat{1}+\hat{L}_{d, \bar{\alpha}}\right] \hat{\mathcal{A}}_{X_{j}}^{\bar{\alpha} \alpha}-\frac{1}{4} \hat{A}_{\alpha}\left[\hat{D}_{Q_{j}}^{\alpha \bar{\alpha}}, \hat{\mathcal{A}}_{Q_{k}}^{\alpha \bar{\alpha}}\right]\left[\hat{D}_{X_{k}}^{\bar{\alpha}}, L_{d, \alpha}^{(0)} \hat{1}+\hat{L}_{d, \bar{\alpha}}\right] \hat{\mathcal{A}}_{X_{j}}^{\bar{\alpha} \alpha} \\
& -\frac{1}{4} \hat{A}_{\alpha} \hat{\mathcal{A}}_{Q_{k}}^{\alpha \bar{\alpha}}\left[\hat{D}_{Q_{j}}^{\alpha},\left[\hat{D}_{X_{k}}^{\bar{\alpha}}, L_{d, \alpha}^{(0)} \hat{1}+\hat{L}_{d, \bar{\alpha}}\right]\right] \hat{\mathcal{A}}_{X_{j}}^{\bar{\alpha} \alpha}-\frac{1}{4}\left[\hat{D}_{Q_{j}}^{\alpha},\left[\hat{D}_{Q_{k}}^{\alpha}, \hat{A}_{\alpha}\right]\right] \hat{\mathcal{A}}_{X_{k}}^{\alpha \bar{\alpha}}\left(L_{d, \alpha}^{(0)} \hat{1}-\hat{L}_{d, \bar{\alpha}}\right) \hat{\mathcal{A}}_{X_{j}}^{\bar{\alpha} \alpha} \\
& -\frac{1}{4}\left[\hat{D}_{Q_{k}}^{\alpha}, \hat{A}_{\alpha}\right]\left[\hat{D}_{Q_{j}}^{\alpha \bar{\alpha}}, \hat{\mathcal{A}}_{X_{k}}^{\alpha \bar{\alpha}}\right]\left(L_{d, \alpha}^{(0)} \hat{1}-\hat{L}_{d, \bar{\alpha}}\right) \hat{\mathcal{A}}_{X_{j}}^{\bar{\alpha} \alpha}-\frac{1}{4}\left[\hat{D}_{Q_{k}}^{\alpha}, \hat{A}_{\alpha}\right] \hat{\mathcal{A}}_{X_{k}}^{\alpha \bar{\alpha}}\left[\hat{D}_{Q_{j}}^{\bar{\alpha}}, L_{d, \alpha}^{(0)} \hat{1}-\hat{L}_{d, \bar{\alpha}}\right] \hat{\mathcal{A}}_{X_{j}}^{\bar{\alpha} \alpha}
\end{aligned}
$$




$$
\begin{aligned}
& -\frac{1}{4} \hat{A}_{\alpha} \hat{\mathcal{A}}_{Q_{k}}^{\alpha \bar{\alpha}}\left[\hat{D}_{X_{k}}^{\bar{\alpha}}, L_{d, \alpha}^{(0)} \hat{1}+\hat{L}_{d, \bar{\alpha}}\right]\left[\hat{D}_{Q_{j}}^{\bar{\alpha}},\left(L_{d, \alpha}^{(0)} \hat{1}-\hat{L}_{d, \bar{\alpha}}\right)^{-1}\right]\left(L_{d, \alpha}^{(0)} \hat{1}-\hat{L}_{d, \bar{\alpha}}\right) \hat{\mathcal{A}}_{X_{j}}^{\bar{\alpha} \alpha} \\
& +\frac{1}{4}\left[\hat{D}_{Q_{k}}^{\alpha}, \hat{A}_{\alpha}\right] \hat{\mathcal{A}}_{X_{k}}^{\alpha \bar{\alpha}}\left(\hat{L}_{d, \bar{\alpha}}-L_{d, \alpha}^{(0)} \hat{1}\right)\left[\hat{D}_{Q_{j}}^{\bar{\alpha}},\left(L_{d, \alpha}^{(0)} \hat{1}-\hat{L}_{d, \bar{\alpha}}\right)^{-1}\right]\left(L_{d, \alpha}^{(0)} \hat{1}-\hat{L}_{d, \bar{\alpha}}\right) \hat{\mathcal{A}}_{X_{j}}^{\bar{\alpha} \alpha} . \\
& -\left\{X_{j} \leftrightarrow Q_{j} \mid X_{k}, Q_{k}\right\}-\left\{X_{j}, Q_{j} \mid X_{k} \leftrightarrow Q_{k}\right\}+\left\{X_{j} \leftrightarrow Q_{j} \mid X_{k} \leftrightarrow Q_{k}\right\}-\text { h.c. }+\mathcal{O}\left(\lambda^{3}\right),
\end{aligned}
$$

and

$$
\begin{aligned}
& \hat{F}_{\alpha}^{(3)}\left(\hat{A}_{\alpha}\right)=\frac{1}{4} \hat{\mathcal{A}}_{Q_{j}}^{\alpha \bar{\alpha}} \hat{\mathcal{A}}_{X_{k}}^{\bar{\alpha} \alpha} \hat{A}_{\alpha} \hat{\mathcal{A}}_{Q_{k}}^{\alpha \bar{\alpha}}\left(L_{d, \alpha}^{(0)} \hat{1}-\hat{L}_{d, \bar{\alpha}}\right) \hat{\mathcal{A}}_{X_{j}}^{\bar{\alpha} \alpha} \\
& \quad-\left\{X_{j} \leftrightarrow Q_{j} \mid X_{k}, Q_{k}\right\}-\left\{X_{j}, Q_{j} \mid X_{k} \leftrightarrow Q_{k}\right\} \\
& \quad+\left\{X_{j} \leftrightarrow Q_{j} \mid X_{k} \leftrightarrow Q_{k}\right\}-\text { h.c. }+\mathcal{O}\left(\lambda^{3}\right) .
\end{aligned}
$$

Eqs. (50), (55) and (56) in combination with eq. (47) are all the terms that enter into the right hand side of eq. (46)) up to $\mathcal{O}\left(\lambda_{1}^{2}, \lambda_{1} \lambda_{2}, \lambda_{2}^{2}\right)$. .

Now that we have obtained an explicit form of eq. (46), we shall next find out a $2 \times 2$ matrix $\hat{A}_{\alpha}$ that satisfies this equation. Before doing this, however, it would be clearly helpful to simplify these more than 100 terms. In fact, we can further transform all these terms precisely into eqs. (67, 69), whose transparent expression helps us to find $\hat{A}_{\alpha}$ in sec. IV. To be more specific, we can find, for any single term in eqs. (50155156), several counterpart terms with which it constitutes a certain $S U(2)$ gauge covariant quantity enumerated in eq. (67,69).

Terms proportional to $\left[\hat{D}_{Q}^{\alpha}, \hat{A}_{\alpha}\right]$ To see this explicitly, focus first on those terms in eqs. (50]55156) that are linear in $\left[\hat{D}^{\alpha}, \hat{A}_{\alpha}\right]$;

$$
\begin{aligned}
\frac{1}{8}\left\{\mathcal{A}_{X_{k}}^{\alpha \bar{\alpha}}\left[D_{X_{j}}^{\bar{\alpha}}, L_{d, \alpha}^{(0)} \hat{1}-\hat{L}_{d, \bar{\alpha}}\right]+\mathcal{A}_{X_{j}}^{\alpha \bar{\alpha}}\left[D_{X_{k}}^{\bar{\alpha}}, L_{d, \alpha}^{(0)} \hat{1}-\hat{L}_{d, \bar{\alpha}}\right]\right. \\
\left.+\left[D_{X_{j}}^{\alpha \bar{\alpha}}, \hat{\mathcal{A}}_{X_{k}}^{\alpha \bar{\alpha}}\right]\left(L_{d, \alpha}^{(0)} \hat{1}-\hat{L}_{d, \bar{\alpha}}\right)\right\} \\
\quad \times\left\{\hat{\mathcal{A}}_{Q_{k}}^{\bar{\alpha} \alpha}\left[D_{Q_{j}}^{\alpha}, \hat{A}_{\alpha}\right]+\hat{\mathcal{A}}_{Q_{j}}^{\bar{\alpha} \alpha}\left[D_{Q_{k}}^{\alpha}, \hat{A}_{\alpha}\right]\right\} \\
\quad+\frac{1}{2}\left(\partial_{X_{j}} L_{d, \alpha}^{(0)}\right)\left[\hat{D}_{Q_{k}}^{\alpha}, \hat{A}_{\alpha}\right] \hat{\mathcal{A}}_{X_{k}}^{\alpha \bar{\alpha}} \hat{\mathcal{A}}_{Q_{j}}^{\bar{\alpha} \alpha} \\
\quad-\frac{1}{4}\left[\hat{D}_{Q_{j}}^{\alpha}, \hat{A}_{\alpha}\right] \hat{\mathcal{A}}_{Q_{k}}^{\alpha \bar{\alpha}}\left[\hat{D}_{X_{k}}^{\bar{\alpha}}, L_{d, \alpha}^{(0)} \hat{1}+\hat{L}_{d, \bar{\alpha}}\right] \hat{\mathcal{A}}_{X_{j}}^{\bar{\alpha} \alpha} \\
\quad-\frac{1}{4}\left[\hat{D}_{Q_{k}}^{\alpha}, \hat{A}_{\alpha}\right]\left[\hat{D}_{Q_{j}}^{\alpha \bar{\alpha}_{j}}, \hat{\mathcal{A}}_{X_{k}}^{\alpha \bar{\alpha}}\right]\left(L_{d, \alpha}^{(0)} \hat{1}-\hat{L}_{d, \bar{\alpha}}\right) \hat{\mathcal{A}}_{X_{j}}^{\bar{\alpha} \alpha} \\
\quad+\cdots .
\end{aligned}
$$

where we have already used $\left[\hat{D}_{Q}^{\bar{\alpha}},\left(L_{d, \alpha}^{(0)} \hat{1}-\hat{L}_{d, \bar{\alpha}}\right)^{-1}\right]$. $\left(L_{d, \alpha}^{(0)} \hat{1}-\hat{L}_{d, \bar{\alpha}}\right)=-\left(L_{d, \alpha}^{(0)} \hat{1}-\hat{L}_{d, \bar{\alpha}}\right)^{-1} \cdot\left[\hat{D}_{Q}^{\bar{\alpha}},\left(L_{d, \alpha}^{(0)} \hat{1}-\hat{L}_{d, \bar{\alpha}}\right)\right]$. Note also that "..." above indicates those 3 kinds of counterpart terms with $X$ and $Q$ exchanged and their hermite conjugate terms;

$$
\begin{aligned}
“ \ldots " \equiv & -\left\{X_{j} \leftrightarrow Q_{j} \mid X_{k}, Q_{k}\right\}-\left\{X_{j}, Q_{j} \mid X_{k} \leftrightarrow Q_{k}\right\} \\
& +\left\{X_{j} \leftrightarrow Q_{j} \mid X_{k} \leftrightarrow Q_{k}\right\} \text { - h.c. }
\end{aligned}
$$

When taking these terms implicit into account, we may rewrite the 1st term in Eq.(157) into the following;

$$
\frac{1}{8}\left\{\mathcal{A}_{X_{k}}^{\alpha \bar{\alpha}}\left[\hat{D}_{X_{j}}^{\bar{\alpha}}, L_{d, \alpha}^{(0)} \hat{1}-\hat{L}_{d, \bar{\alpha}}\right]+\mathcal{A}_{X_{j}}^{\alpha \bar{\alpha}}\left[\hat{D}_{X_{k}}^{\bar{\alpha}}, L_{d, \alpha}^{(0)} \hat{1}-\hat{L}_{d, \bar{\alpha}}\right]\right.
$$

$$
\begin{aligned}
&+ {\left.\left[\hat{D}_{X_{j}}^{\alpha \bar{\alpha}}, \hat{\mathcal{A}}_{X_{k}}^{\alpha \bar{\alpha}}\right]\left(L_{d, \alpha}^{(0)} \hat{1}-\hat{L}_{d, \bar{\alpha}}\right)\right\} } \\
& \times\left\{\hat{\mathcal{A}}_{Q_{k}}^{\bar{\alpha} \alpha}\left[D_{Q_{j}}^{\alpha}, \hat{A}_{\alpha}\right]+\hat{\mathcal{A}}_{Q_{j}}^{\bar{\alpha} \alpha}\left[D_{Q_{k}}^{\alpha}, \hat{A}_{\alpha}\right]\right\}+\cdots \\
&=- \frac{1}{4}\left[\hat{D}_{Q_{j}}^{\alpha}, \hat{A}_{\alpha}\right] \hat{\mathcal{A}}_{Q_{k}}^{\alpha \bar{\alpha}}\left[\hat{D}_{X_{k}}^{\bar{\alpha}}, L_{d, \alpha}^{(0)} \hat{1}-\hat{L}_{d, \bar{\alpha}}\right] \mathcal{A}_{X_{j}}^{\bar{\alpha} \alpha} \\
&+ \frac{1}{4} \mathcal{A}_{X_{k}}^{\alpha \bar{\alpha}}\left[\hat{D}_{X_{j}}^{\bar{\alpha}}, L_{d, \alpha}^{(0)} \hat{1}-\hat{L}_{d, \bar{\alpha}}\right] \hat{\mathcal{A}}_{Q_{k}}^{\bar{\alpha} \alpha}\left[\hat{D}_{Q_{j}}^{\alpha}, \hat{A}_{\alpha}\right] \\
&+\frac{1}{4}\left[\hat{D}_{X_{j}}^{\alpha \bar{\alpha}}, \hat{\mathcal{A}}_{X_{k}}^{\alpha \bar{\alpha}}\right]\left(L_{d, \alpha}^{(0)} \hat{1}-\hat{L}_{d, \bar{\alpha}}\right) \hat{\mathcal{A}}_{Q_{k}}^{\bar{\alpha} \alpha}\left[\hat{D}_{Q_{j}}^{\alpha}, \hat{A}_{\alpha}\right]+\cdots
\end{aligned}
$$

Namely, we replaced several terms in the left hand side by either their counterparts or their hermitian conjugates implicit in "...". We also used eq. (42), only to obtain the $3 \mathrm{rd}$ term in the right hand side. Since we can regard that these terms were just swapped among their 3 other copies and their hermtian conjugates, we can begin with the following, instead of eq. (57);

Eq. (57) =

$$
\begin{aligned}
& \frac{1}{2}\left(\partial_{X_{j}} L_{d, \alpha}^{(0)}\right)\left[D_{Q_{k}}^{\alpha}, \hat{A}_{\alpha}\right]\left(\hat{\mathcal{A}}_{X_{k}}^{\alpha \bar{\alpha}} \hat{\mathcal{A}}_{Q_{j}}^{\bar{\alpha} \alpha}-\hat{\mathcal{A}}_{Q_{j}}^{\alpha \bar{\alpha}} \hat{\mathcal{A}}_{X_{k}}^{\bar{\alpha} \alpha}\right) \\
& +\frac{1}{4} \hat{\mathcal{A}}_{X_{j}}^{\alpha \bar{\alpha}}\left(L_{d, \alpha}^{(0)} \hat{1}-\hat{L}_{d, \bar{\alpha}}\right)\left[D_{Q_{j}}^{\bar{\alpha} \alpha}, \hat{\mathcal{A}}_{X_{k}}^{\bar{\alpha} \alpha}\right]\left[D_{Q_{k}}^{\alpha}, \hat{A}_{\alpha}\right] \\
& +\frac{1}{4} \hat{\mathcal{A}}_{X_{k}}^{\alpha \bar{\alpha}}\left[D_{X_{j}}^{\bar{\alpha}}, L_{d, \alpha} \hat{1}-\hat{L}_{d, \bar{\alpha}}\right] \hat{\mathcal{A}}_{Q_{k}}^{\bar{\alpha} \alpha}\left[D_{Q_{j}}^{\alpha}, \hat{A}_{\alpha}\right] \\
& +\frac{1}{4}\left[D_{X_{j}}^{\alpha \bar{\alpha}}, \hat{\mathcal{A}}_{X_{k}}^{\alpha \bar{\alpha}}\right]\left(L_{d, \alpha}^{(0)} \hat{1}-\hat{L}_{d, \bar{\alpha}}\right) \hat{\mathcal{A}}_{Q_{k}}^{\bar{\alpha} \alpha}\left[D_{Q_{j}}^{\alpha}, \hat{A}_{\alpha}\right] \\
& +\cdots,
\end{aligned}
$$

where "..." defined in eq. (58).

Compare the 2nd, 3rd and 4-th terms above with the decomposition rule for derivatives, i.e. eq. (43). Namely, we can unify these 3 into a single $\alpha$-th band covariant derivative term;

$$
\begin{aligned}
& \text { Eq. (57) }=-\frac{i}{2}\left(\partial_{X_{j}} L_{d, \alpha}^{(0)}\right)\left[D_{Q_{k}}^{\alpha}, \hat{A}_{\alpha}\right] \hat{\Omega}_{Q_{j} X_{k}}^{\alpha} \\
& +\frac{1}{4}\left[\hat{D}_{X_{j}}^{\alpha}, \hat{\mathcal{A}}_{X_{k}}^{\alpha \bar{\alpha}}\left(L_{d, \alpha}^{(0)} \hat{1}-\hat{L}_{d, \bar{\alpha}}\right) \hat{\mathcal{A}}_{Q_{k}}^{\bar{\alpha} \alpha}\right]\left[D_{Q_{j}}^{\alpha}, \hat{A}_{\alpha}\right]+\cdots .
\end{aligned}
$$

When combined explicitly with its counterpart with $X_{k}$ and $Q_{k}$ exchanged, the 2nd term above is expressed by $\hat{\mathcal{M}}_{\alpha}$ (see eq. (48)). Accordingly, eq. (57) can be rigorously transformed into the following $S U(2)$ covariant quantities,

$$
\begin{aligned}
& \text { Eq. (57) }=-\frac{i}{2}\left(\partial_{X_{j}} L_{d, \alpha}^{(0)}\right)\left[\hat{\Omega}_{Q_{j} X_{k}}^{\alpha},\left[\hat{D}_{Q_{k}}^{\alpha}, \hat{A}_{\alpha}\right]\right]_{+} \\
& -\left\{X_{j} \leftrightarrow Q_{j} \mid X_{k}, Q_{k}\right\}-\left\{X_{j}, Q_{j} \mid X_{k} \leftrightarrow Q_{k}\right\} \\
& +\left\{X_{j} \leftrightarrow Q_{j} \mid X_{k} \leftrightarrow Q_{k}\right\} \\
& -\frac{i}{2}\left[\left[\hat{D}_{X_{j}}^{\alpha}, \hat{\mathcal{M}}_{\alpha}\right],\left[\hat{D}_{Q_{j}}^{\alpha}, \hat{A}_{\alpha}\right]\right]_{+}-\left\{Q_{j} \leftrightarrow X_{j}\right\} .
\end{aligned}
$$


Terms proportional to $\hat{A}_{\alpha}$ Employing a similar manipulation, we can further simplify all the remaining terms, which are linear in either (a) $\hat{A}_{\alpha}$ itself or (b) $\left[D_{Q_{j}}^{\alpha},\left[D_{Q_{k}}^{\alpha}, \hat{A}_{\alpha}\right]\right]$. As will be shown next, the latter one can be easily proved to be zero in total up to the order of $\mathcal{O}\left(\lambda^{2}\right)$. Thus, we will henceforth look into those terms in eqs. (50155556), which are proportional to $\hat{A}_{\alpha}$;

$$
\begin{aligned}
& -\frac{1}{8}\left\{\hat{\mathcal{A}}_{X_{j}}^{\alpha \bar{\alpha}}\left(L_{d, \alpha}^{(0)} \hat{1}-\hat{L}_{d, \bar{\alpha}}\right) \hat{\mathcal{A}}_{X_{k}}^{\bar{\alpha} \alpha}+\left(X_{j} \leftrightarrow X_{k}\right)\right\} \\
& \times\left\{\hat{\mathcal{A}}_{Q_{j}}^{\alpha \bar{\alpha}} \hat{\mathcal{A}}_{Q_{k}}^{\bar{\alpha} \alpha} \hat{A}_{\alpha}+\hat{A}_{\alpha} \hat{\mathcal{A}}_{Q_{k}}^{\alpha \bar{\alpha}} \hat{\mathcal{A}}_{Q_{j}}^{\bar{\alpha} \alpha}\right\} \\
& +\frac{1}{8}\left\{\mathcal{A}_{X_{k}}^{\alpha \bar{\alpha}}\left[D_{X_{j}}^{\bar{\alpha}}, L_{d, \alpha}^{(0)} \hat{1}-\hat{L}_{d, \bar{\alpha}}\right]+\left(X_{j} \leftrightarrow X_{k}\right)\right\} \\
& \quad \times\left[D_{Q_{j}}^{\bar{\alpha} \alpha}, \hat{\mathcal{A}}_{Q_{k}}^{\bar{\alpha} \alpha} \hat{A}_{\alpha}\right. \\
& -\frac{1}{4} \hat{A}_{\alpha}\left[\hat{D}_{Q_{j}}^{\alpha \bar{\alpha}}, \hat{\mathcal{A}}_{Q_{k}}^{\alpha \bar{\alpha}}\right]\left[\hat{D}_{X_{k}}^{\bar{\alpha}}, L_{d, \alpha}^{(0)} \hat{1}+\hat{L}_{d, \bar{\alpha}}\right] \hat{\mathcal{A}}_{X_{j}}^{\bar{\alpha} \alpha} \\
& -\frac{1}{4} \hat{A}_{\alpha} \hat{\mathcal{A}}_{Q_{k}}^{\alpha \bar{\alpha}}\left[\hat{D}_{Q_{j}}^{\bar{\alpha}},\left[\hat{D}_{X_{k}}^{\bar{\alpha}}, L_{d, \alpha}^{(0)} \hat{1}+\hat{L}_{d, \bar{\alpha}}\right]\right] \hat{\mathcal{A}}_{X_{j}}^{\bar{\alpha} \alpha} \\
& +\frac{1}{4} \hat{\mathcal{A}}_{Q_{j}}^{\alpha \bar{\alpha}} \hat{\mathcal{A}}_{X_{k}}^{\bar{\alpha} \alpha} \hat{A}_{\alpha} \hat{\mathcal{A}}_{Q_{k}}^{\alpha \bar{\alpha}}\left(L_{d, \alpha}^{(0)} \hat{1}-\hat{L}_{d, \bar{\alpha}}\right) \hat{\mathcal{A}}_{X_{j}}^{\bar{\alpha} \alpha}+\cdots,
\end{aligned}
$$

where "..." defined in eq. (58). We have already neglected those which are canceled either by their counterparts or by their hermite conjugates.

Notice first that, to 2 nd order in $\lambda$, we can regard that $\hat{A}_{\alpha}$ in eq. (61) commutes with other $2 \times 2$ matrices. This is because $\hat{A}_{\alpha}$ reduces to a unit matrix at equilibrium, while eq. (61) is already at least 2nd order in $\lambda_{1}$. Thus, commutators between $\hat{A}_{\alpha}$ and other 2 by 2 matrices inevitably end up with the 3rd order contributions in $\lambda$, e.g.

$$
\begin{aligned}
& \left(\partial_{X_{k}} L_{d, \alpha}^{(0)}\right) \hat{A}_{\alpha}\left[\hat{D}_{Q_{k}}^{\alpha \bar{\alpha}}, \hat{\mathcal{A}}_{X_{j}}^{\alpha \bar{\alpha}}\right] \hat{\mathcal{A}}_{Q_{j}}^{\bar{\alpha} \alpha} \\
& \quad=\left(\partial_{X_{k}} L_{d, \alpha}^{(0)}\right)\left[\hat{D}_{Q_{k}}^{\alpha \bar{\alpha}}, \hat{\mathcal{A}}_{X_{j}}^{\alpha \bar{\alpha}}\right] \hat{\mathcal{A}}_{Q_{j}}^{\bar{\alpha} \alpha} \hat{A}_{\alpha}+\mathcal{O}\left(\lambda_{1}^{2} \lambda_{2}, \lambda_{1}^{3}\right) .
\end{aligned}
$$

Observing this, one can easily unify the 2nd term and (the hermitian conjugate of) 3rd term in eq. (61), so that they are given solely in terms of the Berry's curvature for the $\alpha$-th band,

$$
\begin{gathered}
\frac{1}{8}\left\{\mathcal{A}_{X_{k}}^{\alpha \bar{\alpha}}\left[D_{X_{j}}^{\bar{\alpha}}, L_{d, \alpha}^{(0)} \hat{1}-\hat{L}_{d, \bar{\alpha}}\right]+\left(X_{j} \leftrightarrow X_{k}\right)\right\}\left[D_{Q_{j}}^{\bar{\alpha} \alpha}, \hat{\mathcal{A}}_{Q_{k}}^{\bar{\alpha} \alpha}\right] \hat{A}_{\alpha} \\
+\frac{1}{4} \hat{\mathcal{A}}_{X_{j}}^{\alpha \bar{\alpha}}\left[\hat{D}_{X_{k}}^{\bar{\alpha}}, L_{d, \alpha}^{(0)} \hat{1}+\hat{L}_{d, \bar{\alpha}}\right]\left[\hat{D}_{Q_{j}}^{\bar{\alpha} \alpha}, \hat{\mathcal{A}}_{Q_{k}}^{\bar{\alpha} \alpha}\right] \hat{A}_{\alpha} \\
=\frac{1}{2}\left(\partial_{X_{k}} L_{d, \alpha}^{(0)}\right) \hat{\mathcal{A}}_{X_{j}}^{\alpha \bar{\alpha}}\left[\hat{D}_{Q_{k}}^{\bar{\alpha} \alpha}, \hat{A}_{Q_{j}}^{\bar{\alpha} \alpha}\right] \hat{A}_{\alpha} .
\end{gathered}
$$

Namely, when combined with its counterpart with $X_{j}$ and $Q_{j}$ exchanged and their hermitian conjugate, $\mathcal{O}\left(\lambda^{2}\right)$ contribution of the right hand side above can be expressed only by $\hat{\Omega}_{X_{j} Q_{j}}^{\alpha}$;

$$
\begin{aligned}
& \frac{1}{2}\left(\partial_{X_{k}} L_{d, \alpha}^{(0)}\right)\left\{\hat{\mathcal{A}}_{X_{j}}^{\alpha \bar{\alpha}}\left[\hat{D}_{Q_{k}}^{\bar{\alpha} \alpha}, \hat{A}_{Q_{j}}^{\bar{\alpha} \alpha}\right] \hat{A}_{\alpha}+\hat{A}_{\alpha}\left[\hat{D}_{Q_{k}}^{\alpha \bar{\alpha}}, \hat{A}_{X_{j}}^{\alpha \bar{\alpha}}\right] \hat{\mathcal{A}}_{Q_{j}}^{\bar{\alpha} \alpha}\right\} \\
& \quad-\left\{X_{j} \leftrightarrow Q_{j} \mid X_{k}, Q_{k}\right\} \\
& \quad=\frac{i}{2}\left(\partial_{X_{k}} L_{d, \alpha}^{(0)}\right)\left[\hat{D}_{Q_{k}}^{\alpha}, \hat{\Omega}_{X_{j} Q_{j}}^{\alpha}\right] \hat{A}_{\alpha}+\mathcal{O}\left(\lambda^{3}\right)
\end{aligned}
$$

where we used eqs. 62 63/44).

The 1st, 4-th and 5-th terms in eq. (61) are described in terms of $\hat{\mathcal{M}}_{\alpha}$ and $\hat{\Omega}_{X_{j} Q_{j}}$ in total. To see this, let us begin with the 4 th term;

$$
\begin{aligned}
- & \frac{1}{4} \hat{A}_{\alpha} \hat{\mathcal{A}}_{Q_{k}}^{\alpha \bar{\alpha}}\left[\hat{D}_{Q_{j}}^{\bar{\alpha}},\left[\hat{D}_{X_{k}}^{\bar{\alpha}}, L_{d, \alpha}^{(0)} \hat{1}+\hat{L}_{d, \bar{\alpha}}\right]\right] \hat{\mathcal{A}}_{X_{j}}^{\bar{\alpha} \alpha}+\cdots \\
= & -\frac{1}{8} \hat{A}_{\alpha} \hat{\mathcal{A}}_{Q_{k}}^{\alpha \bar{\alpha}}\left[\hat{D}_{Q_{j}}^{\bar{\alpha}},\left[\hat{D}_{X_{k}}^{\bar{\alpha}}, L_{d, \alpha}^{(0)} \hat{1}+\hat{L}_{d, \bar{\alpha}}\right]\right] \hat{\mathcal{A}}_{X_{j}}^{\bar{\alpha} \alpha} \\
& +\frac{1}{8} \hat{\mathcal{A}}_{Q_{j}}^{\alpha \bar{\alpha}}\left[\hat{D}_{X_{j}}^{\bar{\alpha}},\left[\hat{D}_{Q_{k}}^{\bar{\alpha}}, L_{d, \alpha}^{(0)} \hat{1}+\hat{L}_{d, \bar{\alpha}}\right]\right] \hat{\mathcal{A}}_{X_{k}}^{\bar{\alpha} \alpha} \hat{A}_{\alpha}+\cdots \\
= & -\frac{1}{8} \hat{A}_{\alpha} \hat{\mathcal{A}}_{Q_{k}}^{\alpha \bar{\alpha}}\left(\left[\hat{D}_{Q_{j}}^{\bar{\alpha}},\left[\hat{D}_{X_{k}}^{\bar{\alpha}}, L_{d, \alpha}^{(0)} \hat{1}+\hat{L}_{d, \bar{\alpha}}\right]\right]\right. \\
& \left.-\left[\hat{D}_{X_{k}}^{\bar{\alpha}},\left[\hat{D}_{Q_{j}}^{\bar{\alpha}}, L_{d, \alpha}^{(0)} \hat{1}+\hat{L}_{d, \bar{\alpha}}\right]\right]\right) \hat{\mathcal{A}}_{X_{j}}^{\bar{\alpha} \alpha}+\mathcal{O}\left(\lambda^{3}\right)+\cdots \\
= & \frac{i}{8} \hat{A}_{\alpha} \hat{\mathcal{A}}_{Q_{k}}^{\alpha \bar{\alpha}}\left[\hat{\Omega}_{X_{k} Q_{j}}^{\bar{\alpha}}, L_{d, \alpha}^{(0)} \hat{1}-\hat{L}_{d, \bar{\alpha}}\right]_{-} \hat{\mathcal{A}}_{X_{j}}^{\bar{\alpha} \alpha}+\cdots \\
= & \frac{1}{8} \hat{A}_{\alpha}\left[\hat{\mathcal{A}}_{Q_{k}}^{\alpha \bar{\alpha}} \hat{\mathcal{A}}_{X_{k}}^{\bar{\alpha} \alpha}, \hat{\mathcal{A}}_{Q_{j}}^{\alpha \bar{\alpha}}\left(L_{d, \alpha}^{(0)} \hat{1}-\hat{L}_{d, \bar{\alpha}}\right) \hat{\mathcal{A}}_{X_{j}}^{\bar{\alpha} \alpha}\right]_{-} \\
- & \frac{1}{8} \hat{A}_{\alpha}\left[\hat{\mathcal{A}}_{Q_{k}}^{\alpha \bar{\alpha}} \hat{\mathcal{A}}_{Q_{j}}^{\bar{\alpha} \alpha}, \hat{\mathcal{A}}_{X_{k}}^{\alpha \bar{\alpha}}\left(L_{d, \alpha}^{(0)} \hat{1}-\hat{L}_{d, \bar{\alpha}}\right) \hat{\mathcal{A}}_{X_{j}}^{\bar{\alpha} \alpha}\right]_{-}+\cdots(64)
\end{aligned}
$$

where "..." stands for 3 other counterparts and their hermitian conjugates. In the 1st equality, we have swapped $1 / 2$ of the 1 st term in the left hand side for its counterpart in (the hermitian conjugates of) $\left\{X_{j} \leftrightarrow Q_{j} \mid X_{k} \leftrightarrow Q_{k}\right\}$. In the 2 nd equality, we have ignored $\mathcal{O}\left(\lambda_{1}^{2} \lambda_{2}, \lambda_{1}^{3}\right)$ - contribution associated with the commutators between $\hat{A}_{\alpha}$ and other $2 \times 2$ matrices. We further used eqs. (39|45), only to reach the final expression.

Within $\mathcal{O}\left(\lambda^{2}\right)$, the 2 nd term in eq. (64) is canceled by the 1 st and 5 th term in eq. (61);

$$
\begin{aligned}
& \text { (1st) }+(4 \text { th })+(5 \text { th }) \text { in eq. (61) } \\
& =\frac{1}{8} \hat{A}_{\alpha}\left[\hat{\mathcal{A}}_{Q_{k}}^{\alpha \bar{\alpha}} \hat{\mathcal{A}}_{X_{k}}^{\bar{\alpha} \alpha}, \hat{\mathcal{A}}_{Q_{j}}^{\alpha \bar{\alpha}}\left(L_{d, \alpha}^{(0)} \hat{1}-\hat{L}_{d, \bar{\alpha}}\right) \hat{\mathcal{A}}_{X_{j}}^{\bar{\alpha} \alpha}\right]_{-} \\
& \quad+\mathcal{O}\left(\lambda^{3}\right)+\cdots .
\end{aligned}
$$

Then notice that, when combined with its 3 other counterparts implicit in "...", the coefficient of $\hat{A}_{\alpha}$ above clearly reduces to the commutator between $\hat{\Omega}_{Q_{k} X_{k}}^{\alpha}$ and $\hat{\mathcal{M}}_{\alpha}$ (see eqs. (44|48)). In combination with eq. (63), this dictates that $\mathcal{O}\left(\lambda^{2}\right)$-contributions in eq. (61) are indeed given only by $\hat{\mathcal{M}}_{\alpha}$ and $\hat{\Omega}_{X_{j} Q_{j}}^{\alpha}$;

$$
\begin{aligned}
& \text { eq. (61) }=\frac{i}{4}\left(\partial_{X_{k}} L_{d, \alpha}^{(0)}\right)\left[\left[\hat{D}_{Q_{k}}^{\alpha}, \hat{\Omega}_{X_{j} Q_{j}}^{\alpha}\right], \hat{A}_{\alpha}\right]_{+} \\
& \quad-\left\{X_{k} \leftrightarrow Q_{k}\right\}-\frac{1}{4}\left[\left[\hat{\mathcal{M}}_{\alpha}, \hat{\Omega}_{X_{j} Q_{j}}^{\alpha}\right]_{-}, \hat{A}_{\alpha}\right]_{+}+\mathcal{O}\left(\lambda^{3}\right) .
\end{aligned}
$$

Terms proportional to $\left[\hat{D}_{Q}^{\alpha},\left[\hat{D}_{Q^{\prime}}^{\alpha}, \hat{A}_{\alpha}\right]\right]$ Those terms in eqs. (50155156) which are linear in the 2nd covariant derivative vanish up to $\mathcal{O}\left(\lambda^{2}\right)$. One can easily see this, by noting that any commutator between $\hat{A}_{\alpha}$ and other matrices in eqs. (50|55156) ends up with $\mathcal{O}\left(\lambda_{1}^{2} \lambda_{2}, \lambda_{1}^{3}\right)$

$$
-\frac{1}{8}\left\{\partial_{X_{j}} \partial_{X_{k}} L_{d, \alpha}^{(0)} \hat{1}+\hat{\mathcal{A}}_{X_{j}}^{\alpha \bar{\alpha}}\left(L_{d, \alpha}^{(0)} \hat{1}-\hat{L}_{d, \bar{\alpha}}\right) \hat{\mathcal{A}}_{X_{k}}^{\bar{\alpha} \alpha}\right.
$$




$$
\begin{aligned}
+ & \left.\hat{\mathcal{A}}_{X_{k}}^{\alpha \bar{\alpha}}\left(L_{d, \alpha}^{(0)} \hat{1}-\hat{L}_{d, \bar{\alpha}}\right) \hat{\mathcal{A}}_{X_{j}}^{\bar{\alpha} \alpha}\right\} \cdot\left[D_{Q_{j}}^{\alpha},\left[D_{Q_{k}}^{\alpha}, \hat{A}_{\alpha}\right]\right] \\
- & \frac{1}{4}\left[\hat{D}_{Q_{j}}^{\alpha},\left[\hat{D}_{Q_{k}}^{\alpha}, \hat{A}_{\alpha}\right]\right] \hat{\mathcal{A}}_{X_{k}}^{\alpha \bar{\alpha}}\left(L_{d, \alpha}^{(0)} \hat{1}-\hat{L}_{d, \bar{\alpha}}\right) \hat{\mathcal{A}}_{X_{j}}^{\bar{\alpha} \alpha}+\cdots \\
=- & \frac{1}{8}\left\{\hat{\mathcal{A}}_{X_{j}}^{\alpha \bar{\alpha}}\left(L_{d, \alpha}^{(0)} \hat{1}-\hat{L}_{d, \bar{\alpha}}\right) \hat{\mathcal{A}}_{X_{k}}^{\bar{\alpha} \alpha}-\left\{X_{k} \leftrightarrow X_{j}\right\}\right\} \\
& \times \partial_{Q_{j}} \partial_{Q_{k}}\left(\hat{A}_{\alpha}\right)+\mathcal{O}\left(\lambda^{3}\right)+\cdots=\mathcal{O}\left(\lambda^{3}\right),
\end{aligned}
$$

where "..." already defined in eq. (58).
3. $S U(2)$ RKE up to the 2nd order accuracy and its physical implications

According to the analyses up to the previous subsubsection such as eqs. (47/60]66), the $\mathcal{O}\left(\lambda^{2}\right)$-contributions of eqs. (50155156) can be rigorously transformed into the following;

$$
\begin{aligned}
\hat{\mathcal{L}}_{1}\left(\hat{A}_{\alpha}\right)+\hat{\mathcal{L}}_{2}\left(\hat{A}_{\alpha}\right) \equiv & {\left[\hat{L}_{d, \alpha}, \hat{A}_{\alpha}\right]+\hat{F}_{\alpha}^{(1)}\left(\hat{A}_{\alpha}\right)+\hat{F}_{\alpha}^{(2)}\left(\hat{A}_{\alpha}\right)+\hat{F}_{\alpha}^{(3)}\left(\hat{A}_{\alpha}\right)+\mathcal{O}\left(\lambda^{3}\right) . } \\
\hat{\mathcal{L}}_{1}\left(\hat{A}_{\alpha}\right)=- & -\left[\hat{\epsilon}_{\alpha}+\hat{\mathcal{M}}_{\alpha}, \hat{A}_{\alpha}\right]_{-}+i\left(\partial_{X_{k}} L_{d, \alpha}^{(0)}\right)\left[\hat{D}_{Q_{k}}^{\alpha}, \hat{A}_{\alpha}\right]-i\left(\partial_{Q_{k}} L_{d, \alpha}^{(0)}\right)\left[\hat{D}_{X_{k}}^{\alpha}, \hat{A}_{\alpha}\right] \\
\hat{\mathcal{L}}_{2}\left(\hat{A}_{\alpha}\right)=- & -\frac{1}{4}\left[\left[\hat{\epsilon}_{\alpha}, \hat{\Omega}_{X_{j}, Q_{j}}^{\alpha}\right]_{-}, \hat{A}_{\alpha}\right]_{+}-\frac{1}{4}\left[\left[\hat{\mathcal{M}}_{\alpha}, \hat{\Omega}_{X_{j}, Q_{j}}^{\alpha}\right]_{-}, \hat{A}_{\alpha}\right]_{+} \\
& +\frac{1}{4}\left[i\left(\partial_{X_{k}} L_{d, \alpha}^{(0)}\right)\left[\hat{D}_{Q_{k}}^{\alpha}, \hat{\Omega}_{X_{j}, Q_{j}}^{\alpha}\right]-i\left(\partial_{Q_{k}} L_{d, \alpha}^{(0)}\right)\left[\hat{D}_{X_{k}}^{\alpha}, \hat{\Omega}_{X_{j}, Q_{j}}^{\alpha}\right], \hat{A}_{\alpha}\right]_{+} \\
& -\frac{i}{2}\left[\left[\hat{D}_{X_{j}}^{\alpha}, \hat{\epsilon}_{\alpha}+\hat{\mathcal{M}}_{\alpha}\right],\left[\hat{D}_{Q_{j}}^{\alpha}, \hat{A}_{\alpha}\right]\right]_{+}+\frac{i}{2}\left[\left[\hat{D}_{Q_{j}}^{\alpha}, \hat{\epsilon}_{\alpha}+\hat{\mathcal{M}}_{\alpha}\right],\left[\hat{D}_{X_{j}}^{\alpha}, \hat{A}_{\alpha}\right]\right]_{+} \\
& -\frac{i}{2}\left(\partial_{X_{k}} L_{d, \alpha}^{(0)}\right)\left[\hat{\Omega}_{Q_{k}, X_{j}}^{\alpha},\left[\hat{D}_{Q_{j}}^{\alpha}, \hat{A}_{\alpha}\right]\right]_{+}+\frac{i}{2}\left(\partial_{Q_{k}} L_{d, \alpha}^{(0)}\right)\left[\hat{\Omega}_{X_{k}, X_{j}}^{\alpha},\left[\hat{D}_{Q_{j}}^{\alpha}, \hat{A}_{\alpha}\right]\right]_{+} \\
& +\frac{i}{2}\left(\partial_{X_{k}} L_{d, \alpha}^{(0)}\right)\left[\hat{\Omega}_{Q_{k}, Q_{j}}^{\alpha},\left[\hat{D}_{X_{j}}^{\alpha}, \hat{A}_{\alpha}\right]\right]_{+}-\frac{i}{2}\left(\partial_{Q_{k}} L_{d, \alpha}^{(0)}\right)\left[\hat{\Omega}_{X_{k}, Q_{j}}^{\alpha},\left[\hat{D}_{X_{j}}^{\alpha}, \hat{A}_{\alpha}\right]\right]_{+} .
\end{aligned}
$$

$\mathcal{L}_{i}$ above stands for the $\mathcal{O}\left(\lambda^{i}\right)$-contribution.Observing these results, notice that the first term in $\hat{\mathcal{L}}_{2}\left(\hat{\mathcal{A}}_{\alpha}\right)$ is nothing but the anti-commutator between $\hat{\mathcal{N}}_{\alpha}$ and $\hat{A}_{\alpha}$, encoded in $\mathcal{O}\left(\lambda_{1}\right)$-contribution of $F^{(1)}\left(\hat{A}_{\alpha}\right)$ (see eqs. (4749) $)$. On the one hand, the 2 nd term in $\hat{\mathcal{L}}_{2}\left(\hat{\mathcal{A}}_{\alpha}\right)$ is obtained from the $\mathcal{O}\left(\lambda_{1}^{2}\right)$-contribution in $\hat{F}^{(1)}, \hat{F}^{(2)}$ and $\hat{F}^{(3)}$, i.e. eq. (66). In spite of these apparently different origins, $\hat{\epsilon}_{\alpha}$ and $\hat{\mathcal{M}}_{\alpha}$ in these first two terms appear in a totally parallel fashion. We can see a similar feature also in the 5 -th and 6 -th term in $\mathcal{L}_{2}\left(\hat{A}_{\alpha}\right)$. These observations not only imply the consistency of our derived $S U(2)$ RKE but also dictate that $\hat{\mathcal{M}}_{\alpha}$ indeed plays role of the $\mathcal{O}\left(\lambda_{1}\right)$-correction to the quasi-particle energy dispersion. Therefore, its non-trivial matrix structure as well as that of $\hat{\epsilon}_{\alpha}$ induces the spin-precession of quasi-particles.

When a disequilibration is created only by the external electromagnetic fields, $\hat{\mathcal{M}}_{\alpha}$ defined in eq. (48) is composed by the spatial (magnetic) component and temporal (electric) one. The former is the Zeeman coupling between an external magnetic field and internal magnetic moment associated quasi-particle wavepacket. The later one is that between an applied electric field and internal electric dipole moment. To see these internal dipole moments explicitly, consider a situation in the presence of physical (i.e. external) electromagnetic gauge fields $\left(\mathrm{a}_{0}, \mathbf{a}\right)$, with corresponding physical electromagnetic fields $\mathbf{b}=\nabla \times \mathbf{a}, \mathbf{e}=\nabla_{R} \mathrm{a}_{0}-\partial_{T} \mathbf{a}$. Then, following the standard recipe, let us introduce the canoni- cal momentum $k \equiv q+\mathbf{a}(T, R)$ and canonical frequency $\omega^{\prime} \equiv \omega-\mathrm{a}_{0}(R) \stackrel{29}{2} ;$

$$
\left|u_{X ; Q}^{\alpha \sigma}\right\rangle \equiv\left|u_{k \equiv q+\mathrm{a}, \omega^{\prime} \equiv \omega-\mathrm{a}_{0}}^{(0), \alpha \sigma}\right\rangle .
$$

Substituting this into eq. (48), one can then re-express $\hat{\mathcal{M}}_{\alpha}$, such that it is given solely by the partial derivative with respect to these canonical quantities. Namely, this $\mathcal{O}\left(\lambda_{1}\right)$-correction to a q.p. energy dispersion precisely reduces into an inner product between external electromagnetic fields and sort of internal dipole moments;

$$
\begin{aligned}
& {\left[\hat{\mathcal{M}}_{\alpha}\right]=\mathbf{b} \cdot\left[\hat{\mathbf{M}}_{\alpha}\right]+\mathbf{e} \cdot\left[\hat{\mathbf{P}}_{\alpha}\right] } \\
& {\left[\hat{\mathbf{M}}_{\alpha, m}\right]_{\sigma \sigma^{\prime}}=} \frac{i \epsilon_{m n l}}{2} \\
& \times\left\langle\partial_{k_{n}} u_{k, \omega^{\prime}}^{(0), \alpha \sigma}\left|L_{d, \alpha}^{(0)}-\hat{\mathrm{L}}\right| \partial_{k_{l}} u_{k, \omega^{\prime}}^{(0), \alpha \sigma^{\prime}}\right\rangle,(70) \\
& {\left[\hat{\mathbf{P}}_{\alpha, m}\right]_{\sigma \sigma^{\prime}} }=\frac{i}{2}\left(\left\langle\partial_{\omega^{\prime}} u_{k, \omega^{\prime}}^{(0), \alpha \sigma}\left|L_{d, \alpha}^{(0)}-\hat{\mathrm{L}}\right| \partial_{k_{m}} u_{k, \omega^{\prime}}^{(0), \alpha \sigma^{\prime}}\right\rangle\right. \\
&\left.-\left\langle\partial_{k_{m}} u_{k, \omega^{\prime}}^{(0), \alpha \sigma}\left|L_{d, \alpha}^{(0)}-\hat{\mathrm{L}}\right| \partial_{\omega^{\prime}} u_{k, \omega^{\prime}}^{(0), \alpha \sigma^{\prime}}\right\rangle\right) .
\end{aligned}
$$

Note that the magnetic dipole moment $\mathbf{M}_{\alpha}$ above reproduces eq. (4) in a non-interacting limit. On the other hand, the electric dipole moment $\mathbf{P}_{\alpha}$ has no noninteracting counterpart, since it is purely associated with the energy-derivative of the quasi-particle Bloch wavefunction.

As is trivial from its coupling with $\mathbf{e}$, when considered in $U(1) \mathrm{FLs}$, this electric dipole moment $\mathbf{P}_{\alpha}$ is literally 
time-reversally even while parity odd;

$$
\begin{aligned}
& \mathbf{P}_{\alpha}(k)=\mathbf{P}_{\alpha}(-k) \quad T \text {-reversal, } \\
& \mathbf{P}_{\alpha}(k)=-\mathbf{P}_{\alpha}(-k) I \text {-invserse. }
\end{aligned}
$$

Therefore, in $S U(2)$ FLs where both of these symmetries are guaranteed, $\mathbf{P}_{\alpha}$ becomes traceless just in a same way as $\mathbf{M}_{\alpha}$ does;

$$
\operatorname{Tr}\left[\mathbf{P}_{\alpha}(k)\right] \equiv \operatorname{Tr}\left[\mathbf{M}_{\alpha}(k)\right] \equiv 0 .
$$

When translated into the the $S U(2)$ effective EOM, these two dipole moments, first of all, enter into that for the $C P^{1}$ vector;

$$
i \frac{d \mathbf{z}}{d t}=\left\{\hat{\mathbf{M}}_{\alpha} \cdot \mathbf{b}+\hat{\mathbf{P}}_{\alpha} \cdot \mathbf{e}+\cdots\right\} \mathbf{z},
$$

which describes the spin-precession due to the external electromagnetic fields. Observing eqs. (67, 69), note also that every derivative term found in a conventional Keldysh equation is now replaced by the corresponding $S U(2)$ covariant derivative in our $S U(2)$ RKE;

$$
\partial_{X}(\cdots) \rightarrow\left[\hat{D}_{X}^{\alpha}, \cdots\right] \equiv \partial_{X}(\cdots)+\left[\hat{\mathcal{A}}_{X}^{\alpha}, \cdots\right] .
$$

Therein, its usual derivative part describes the charge degree of freedom, while the commutator with $\hat{\mathcal{A}}_{X}^{\alpha}$ stands for the precession of quasi-particle spin due to this gauge field. In this sense, our derived RKE treats the charge and spin degrees of freedom on a equal footing way, by using the $S U(2)$ covariant derivative.

To uncover the physical significance of remaining terms in $\hat{\mathcal{L}}_{2}$ such as the 1 st 4 terms and the final 4 terms, we further need to solve this reduced Keldysh equation in $\mathrm{fa}$ vor of $\hat{A}_{\alpha}$ (sec. IV) and to derive the effective Boltzmann equation (sec. V) respectively.

\section{PERTURBATIVE SOLUTION FOR RKES}

\section{A. prescription}

When obtaining the reduced Keldysh equation described in the previous section, we have chosen the spectral function at equilibrium as a unit matrix with its coefficient to be a delta function of $L_{d, \alpha}^{(0)}$;

$$
\hat{A}_{\alpha}=\delta\left(L_{d, \alpha}^{(0)}\right) \hat{1}=Z_{\alpha}^{(0)} \delta\left(\omega-\epsilon_{\alpha}^{(0)}\right) \hat{1} \equiv f_{0}\left(L_{d, \alpha}^{(0)}\right) \hat{1} .
$$

This is not only because such solutions are physically sensible, but also because, starting from this zero-th order spectral functions, we can indeed keep on satisfying the RKE up to higher order in $\lambda$. We will prove this point, by solving actually the derived RKE in favor for $\hat{A}_{\alpha}$ perturbatively in $\lambda$ (see eq. (81)).

Our choice of the spectral function at equilibrium clearly satisfies the derived $S U(2)$ RKE up to the 1st order;

$$
\begin{aligned}
\hat{\mathcal{L}}_{1}\left(f_{0} \hat{1}\right) & =i\left\{\left(\partial_{X_{k}} L_{d, \alpha}^{(0)}\right)\left(\partial_{Q_{k}} L_{d, \alpha}^{(0)}\right)-\left\{X_{k} \leftrightarrow Q_{k}\right\}\right\} f_{0}^{\prime} \hat{1} \\
& =\hat{0} .
\end{aligned}
$$

However, it does not up to the 2 nd order, i.e. $\hat{\mathcal{L}}_{2}\left(f_{0} \hat{1}\right) \neq$ $\hat{0}$. To resolve this, we will introduce the 1 st order correction to the spectral functions;

$$
\hat{A}_{\alpha}=f_{0} \hat{1}+\hat{f}_{1}+\cdots,
$$

such that $\hat{A}_{\alpha}$ satisfies the $S U(2)$ RKEs even up to the 2nd order accuracy;

$$
\hat{0}=\hat{\mathcal{L}}_{1}\left(\hat{f}_{1}\right)+\hat{\mathcal{L}}_{2}\left(f_{0} \hat{1}\right) .
$$

In general, we could further obtain the higher order correction of the spectral function $\hat{A}_{\alpha}$, provided that the RKE is given up to the 3rd order's accuracy or higher than that, i.e. $\hat{\mathcal{L}}=\hat{\mathcal{L}}_{1}+\hat{\mathcal{L}}_{2}+\hat{\mathcal{L}}_{3}+\hat{\mathcal{L}}_{4}+\cdots$. Namely, its 3rd order part $\hat{\mathcal{L}}_{3}$ determines the the 2 nd order correction to $\hat{A}_{\alpha}$ in terms of $\hat{f}_{1}$ and $f_{0}$;

$$
\hat{0}=\hat{\mathcal{L}}_{1}\left(\hat{f}_{2}\right)+\hat{\mathcal{L}}_{2}\left(\hat{f}_{1}\right)+\hat{\mathcal{L}}_{3}\left(f_{0} \hat{1}\right),
$$

The following equations further specify the higher order corrections such as $\hat{f}_{3}, \cdots$, in terms of $\hat{f}_{2}, \hat{f}_{1}$ and $f_{0}$ iteratively;

$$
\begin{aligned}
& \hat{0}=\hat{\mathcal{L}}_{1}\left(\hat{f}_{3}\right)+\hat{\mathcal{L}}_{2}\left(\hat{f}_{2}\right)+\hat{\mathcal{L}}_{3}\left(\hat{f}_{1}\right)+\hat{\mathcal{L}}_{4}\left(f_{0} \hat{1}\right), \\
& \hat{0}=\cdots
\end{aligned}
$$

However, our derived RKE being exact up to $\mathcal{O}\left(\lambda_{1}^{2}, \lambda_{1} \lambda_{2}, \lambda_{2}^{2}\right)$, the highest order to which accuracy we could determine $\hat{A}_{\alpha}$ is $\hat{f}_{1}$.

\section{B. first order correction to $\hat{A}_{\alpha}$}

As we will show below, even up to this lowest order analysis, we obtain a non-trivial correction to the spectral function, having an interesting physical implication (next subsection). Notice first the last 4 terms in eq. (69) do not contribute at all to $\mathcal{L}_{2}\left(f_{0} \hat{1}\right)$, since they are antisymmetrized with respect to the exchange between $X$ and $Q$;

$$
\begin{aligned}
& \mathcal{L}_{2}\left(f_{0} \hat{1}\right)=-\frac{f_{0}}{2}\left\{\left[\hat{\epsilon}_{\alpha}+\hat{\mathcal{M}}_{\alpha}, \hat{\Omega}_{X_{j} Q_{j}}^{\alpha}\right]_{-}+\right. \\
& \left.-i\left(\partial_{X_{k}} L_{d, \alpha}^{(0)}\right)\left[\hat{D}_{Q_{k}}^{\alpha}, \hat{\Omega}_{X_{j} Q_{j}}^{\alpha}\right]+i\left(\partial_{Q_{k}} L_{d, \alpha}^{(0)}\right)\left[\hat{D}_{X_{k}}^{\alpha}, \hat{\Omega}_{X_{j} Q_{j}}^{\alpha}\right]\right\} \\
& -f_{0}^{\prime}\left\{i\left(\partial_{Q_{j}} L_{d, \alpha}^{(0)}\right)\left[\hat{D}_{X_{j}}^{\alpha}, \hat{\epsilon}_{t, \alpha}\right]-i\left(\partial_{X_{j}} L_{d, \alpha}^{(0)}\right)\left[\hat{D}_{Q_{j}}^{\alpha}, \hat{\epsilon}_{t, \alpha}\right]\right\} .
\end{aligned}
$$

where $\hat{\epsilon}_{t, \alpha} \equiv \hat{\epsilon}_{\alpha}+\hat{\mathcal{M}}_{\alpha}$. Then, the last two terms above are readily canceled in eq. (76), when $\hat{f}_{1}=-\hat{\epsilon}_{t, \alpha} f_{0}^{\prime}$ is substituted into $\hat{\mathcal{L}}_{1}$;

$$
\begin{aligned}
& \hat{\mathcal{L}}_{1}\left(-\hat{\epsilon}_{t, \alpha} f_{0}^{\prime}\right)+\hat{\mathcal{L}}_{2}\left(f_{0} \hat{1}\right)= \\
& -\frac{f_{0}}{2}\left\{\left[\hat{\epsilon}_{t, \alpha}, \hat{\Omega}_{X_{j} Q_{j}}^{\alpha}\right]_{-}-i\left(\partial_{X_{k}} L_{d, \alpha}^{(0)}\right)\left[\hat{D}_{Q_{k}}^{\alpha}, \hat{\Omega}_{X_{j} Q_{j}}^{\alpha}\right]\right. \\
& \left.\quad+i\left(\partial_{Q_{k}} L_{d, \alpha}^{(0)}\right)\left[\hat{D}_{X_{k}}^{\alpha}, \hat{\Omega}_{X_{j} Q_{j}}^{\alpha}\right]\right\} .
\end{aligned}
$$


Since $\hat{\epsilon}_{t, \alpha}$ is regarded as a small quantity, $-\hat{\epsilon}_{t, \alpha} f_{0}^{\prime}$ is nothing but the $\mathcal{O}(\lambda)$-correction to the argument of $f_{0}$, i.e. $\hat{L}_{d, \alpha}^{(0)}$.

To set off also those terms in $\hat{\mathcal{L}}_{2}\left(f_{0} \hat{1}\right)$ which are linear in $f_{0}$ itself, we need to introduce a correction to an overall coefficient of $f_{0}$, in addition to that to its argument. Namely, consider the following $\hat{f}_{1}$;

$$
\hat{f}_{1} \equiv-\hat{\epsilon}_{t, \alpha} f_{0}^{\prime}-\frac{1}{2} \hat{\Omega}_{X_{j} Q_{j}}^{\alpha} f_{0}
$$

Then, when entering into $\hat{\mathcal{L}}_{1}$, the 2 nd term above totally cancel all the terms in the right hand side of eq. (79).

Notice that, this cancellation becomes possible, only because the relative ratio among the coefficients of first 3 terms in $\hat{\mathcal{L}}_{2}\left(\hat{A}_{\alpha}\right)$ perfectly match with the ratio among the corresponding three terms in $\hat{\mathcal{L}}_{1}\left(\hat{A}_{\alpha}\right)$;

$$
\begin{aligned}
\hat{\mathcal{L}}_{2}\left(\hat{A}_{\alpha}\right)=- & \frac{1}{4}\left[\left[\hat{\epsilon}_{\alpha}, \hat{\Omega}_{X_{j}, Q_{j}}^{\alpha}\right]_{-}, \hat{A}_{\alpha}\right]_{+} \\
- & \frac{1}{4}\left[\left[\hat{\mathcal{M}}_{\alpha}, \hat{\Omega}_{X_{j}, Q_{j}}^{\alpha}\right]_{-}, \hat{A}_{\alpha}\right]_{+} \\
+ & \frac{i}{4}\left[\left(\partial_{X_{k}} L_{d, \alpha}^{(0)}\right)\left[\hat{D}_{Q_{k}}^{\alpha}, \hat{\Omega}_{X_{j}, Q_{j}}^{\alpha}\right], \hat{A}_{\alpha}\right]_{+} \\
- & \left\{X_{k} \leftrightarrow Q_{k}\right\}+\cdots, \\
\leftrightarrow \quad \hat{\mathcal{L}}_{1}\left(\hat{A}_{\alpha}\right)= & -\left[\hat{\epsilon}_{\alpha}, \hat{A}_{\alpha}\right]_{-}-\left[\hat{\mathcal{M}}_{\alpha}, \hat{A}_{\alpha}\right]_{-} \\
& +i\left(\partial_{X_{k}} L_{d, \alpha}^{(0)}\right)\left[\hat{D}_{Q_{k}}^{\alpha}, \hat{A}_{\alpha}\right]-\left\{X_{k} \leftrightarrow Q_{k}\right\} .
\end{aligned}
$$

If either signs or coefficients of any one of the 3 terms in $\hat{\mathcal{L}}_{2}$ were not to meet with those of corresponding 3 terms in $\hat{\mathcal{L}}_{1}$, we could never have any $\hat{f}_{1}$ satisfying eq. (76). In other words, there is no a priori guarantee for the existence of $\hat{f}_{1}$ which satisfies eq. (76). In spite of this, both signs and coefficients in $\hat{\mathcal{L}}_{2}$ completely met with those in $\hat{\mathcal{L}}_{1}$, we safely have eq. (80) as an appropriate $\mathcal{O}(\lambda)$-correction to $\hat{A}_{\alpha}$. Reversely speaking, this perfect coincidence between $\mathcal{L}_{1}$ and $\mathcal{L}_{2}$ indicates the validity and the consistency of our derived $S U(2)$ RKE.

\section{Physical Implications}

Let us next argue the physical consequence of our solution $\hat{A}_{\alpha}$;

$$
\hat{A}_{\alpha}=f_{0}\left(L_{d, \alpha}^{(0)}\right) \hat{1}-\hat{\epsilon}_{t, \alpha} f_{0}^{\prime}\left(L_{d, \alpha}^{(0)}\right)-\frac{1}{2} \hat{\Omega}_{X_{j} Q_{j}}^{\alpha} f_{0}\left(L_{d, \alpha}^{(0)}\right) .
$$

As was already mentioned, the 2nd term above clearly stands for the renormalization of the energy dispersion. Namely, diagonalizing $\hat{\epsilon}_{\alpha}+\hat{\mathcal{M}}_{\alpha} \equiv \hat{\epsilon}_{t, \alpha}$, we can transcribe this correction into that of the arguments of $f_{0}$,

$f_{0} \hat{1}-\hat{\epsilon}_{t, \alpha} f_{0}^{\prime} \simeq\left[\begin{array}{ll}f_{0}\left(L_{d, \alpha}^{(0)}-\epsilon_{t, \alpha,+}\right) & \\ & f_{0}\left(L_{d, \alpha}^{(0)}-\epsilon_{t, \alpha,-}\right)\end{array}\right]$.

A finite $\epsilon_{t, \alpha,+}-\epsilon_{t, \alpha,-}$ thereby lifts the doubly degeneracy at equilibrium (Fig. 4(b)).
On the other hand, the last term in eq. (81) changes the weight of the spectral function. To see this, let us integrate eq. (81) with respect to frequency;

$$
\int_{-\Lambda}^{\Lambda} \hat{A}_{\alpha} d \omega=Z_{\alpha}^{(0)} \times\left\{1-\frac{1}{2}\left(\hat{\Omega}_{X_{j} Q_{j}}\right)_{\mid \omega=\epsilon_{\alpha}^{(0)}}\right\},
$$

where $Z_{\alpha}^{(0)}$ stands for the residue at $L_{d, \alpha}^{(0)}=0$. This clearly dictates that, apart from the conventional renormalization factor $Z_{\alpha}^{(0)}$, the integrated spectral weight acquires an additional $\mathcal{O}(\lambda)$-correction which is given by the $S U(2)$ Berry's curvature.

This curvature correction $\hat{\Omega}_{X_{j} Q_{j}}^{\alpha}$ has a spin-selective effect. Namely, choosing the basis diagonalizing this $S U(2)$ Berry's curvature, we have,

$\int_{-\Lambda}^{\Lambda} \hat{A}_{\alpha} d \omega \sim Z_{\alpha}^{(0)} \times\left[\begin{array}{cc}1-\frac{1}{2} \Omega_{X_{j} Q_{j}}^{\alpha,+} & 0 \\ 0 & 1-\frac{1}{2} \Omega_{X_{j} Q_{j}}^{\alpha,-}\end{array}\right]_{\mid \omega=\epsilon_{\alpha}^{(0)}}$

where the up-chirality electron and down one generally acquire the different correction to its spectral weight with each other.

Observing eq. (83), we can propose several photoemission experiments as a candidate experimental tool to visualize the dual $S U(2)$ electromagnetic fields in a momentum resolved way. To see this, consider the situation in the presence of applied electromagnetic fields, i.e. $\mathbf{b}=\nabla \times \mathbf{a}$ and $\mathbf{e}=\nabla \mathrm{a}_{0}-\partial_{T} \mathbf{a}$. Then, using the canonical momentum and frequency introduced previously, eq. (82) reduces into the vector product between the real electromagnetic fields and the a sort of dual version of $S U(2)$ electromagnetic fields;

$$
\begin{aligned}
Z_{\alpha} & \equiv Z_{\alpha}^{(0)} \times\left\{1-\frac{1}{2}\left(\hat{\Omega}_{X_{j} Q_{j}}^{\alpha}\right)_{\mid \omega=\epsilon_{\alpha}^{(0)}}\right\} \\
& =Z_{\alpha}^{(0)} \times\left\{1+\frac{1}{2} \overline{\mathcal{B}}^{\alpha} \cdot \mathbf{b}+\frac{1}{2} \overline{\mathcal{E}}^{\alpha} \cdot \mathbf{e}\right\}
\end{aligned}
$$

Here the dual quantities in the right hand side are estimated on shell at equilibrium;

$$
\begin{aligned}
\overline{\mathcal{B}}_{m}^{\alpha}= & i \epsilon_{m n l}\left\{\partial_{k_{n}} \mathcal{A}_{l}^{\alpha}+\mathcal{A}_{n}^{\alpha} \mathcal{A}_{l}^{\alpha}\right\}_{\mid \omega=\epsilon_{\alpha}^{(0)}}, \\
\overline{\mathcal{E}}_{m}^{\alpha}= & i\left\{\partial_{0} \mathcal{A}_{m}^{\alpha}-\partial_{m} \mathcal{A}_{0}^{\alpha}+\left[\mathcal{A}_{0}^{\alpha}, \mathcal{A}_{m}^{\alpha}\right]\right\}_{\mid \omega=\epsilon_{\alpha}^{(0)}}, \\
& {\left[\mathcal{A}_{m}^{\alpha}\right]_{\sigma \sigma^{\prime}} \equiv\left\langle u_{k, \omega}^{(0), \alpha \sigma} \mid \partial_{k_{m}} u_{k, \omega}^{(0), \alpha \sigma^{\prime}}\right\rangle, } \\
& {\left[\mathcal{A}_{0}^{\alpha}\right]_{\sigma \sigma^{\prime}} \equiv\left\langle u_{k, \omega}^{(0), \alpha \sigma} \mid \partial_{\omega} u_{k, \omega}^{(0), \alpha \sigma^{\prime}}\right\rangle, }
\end{aligned}
$$

with $m=1, \cdots d$.

As is clear from their coupling with real electromagnetic fields, $\overline{\mathcal{B}}_{m}^{\alpha}(k)$ and $\overline{\mathcal{E}}_{m}^{\alpha}(k)$ as functions of $k$ obey precisely same symmetries as $\mathbf{M}_{\alpha, m}(k)$ and $\mathbf{P}_{\alpha, m}(k)$ do respectively. Thus, in a $S U(2)$ FL having time-reversal and spatial inversion symmetry, these $S U(2)$ matrices become both traceless;

$$
\operatorname{Tr}\left[\overline{\mathcal{E}}_{m}^{\alpha}(k)\right] \equiv \operatorname{Tr}\left[\overline{\mathcal{B}}_{m}^{\alpha}(k)\right] \equiv 0
$$




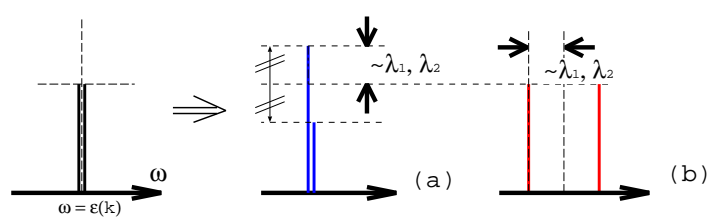

FIG. 4: A schematic picture of spectral functions in $S U(2)$ case. (a): The correction due to the Berry's curvature changes its weight. Furthermore, the change for the up-spin weight and that for the down-spin are in general different. (b): Usual 1st order correction change an energy spectrum.

just as in eq. (72). This suggests that the (integrated) spectral weights for the doubly degenerate quasiparticles, subjected under applied electromagnetic fields, acquire the 1st order corrections having a reverse sign with each other (see Fig. 廿4(a)).

The spectral weight for quasi-particle is in principle detectable in a momentum resolved way in terms of photoemission experiments such as angle resolved photoemission spectroscopy (ARPES) and resonating inelastic X-ray scattering (RIXS). The above theoretical observation therefore leads us to raise the spin-resolved ARPES as the potential candidate tool to measure the dual $S U(2)$ electromagnetic fields. Namely, observing the change of the spin-resolved spectral weight at each $k$-point under small $\mathbf{e}$ or $\mathbf{b}$, we can identify the dual electric or magnetic field as its linear response. Furthermore, eq. (87) claims that, maximizing the measured linear responses with respect to the resolved-spin's direction, one can even determine the spin-quantization axis for the eigenbasis diagonalizing $\overline{\mathcal{B}}^{\alpha}$ or $\overline{\mathcal{E}}^{\alpha}$. Thus, provided that these processes are performed for all the components of $\mathbf{e}$ and $\mathbf{b}$ separately, we could determine, as 2 by 2 matrices, all the $\mathbf{2} \times \mathbf{d}$ components of these $S U(2)$ Berry's curvatures at an arbitrary $k$ point on a Fermi surface.

The (spin-resolved) ARPES experiment under external electromagnetic fields is, however, a difficult experiment. A more relatively less unrealistic proposal might be detecting abelian Berry's curvatures in $U(1)$ FLs, such as ferromagnetic metals or paramagnetic metals without any centrosymmetric lattice point. In such $U(1)$ FLs, we have only to regard all the $S U(2)$ hermitian matrices in eqs. 67]69) as a scalar quantity, to obtain the $U(1)$ RKEs and its solutions up to the 1st order accuracy 30 ;

$$
\begin{aligned}
A_{\alpha} & =\left(1-\frac{1}{2} \Omega_{X_{j} Q_{j}}^{\alpha}\right) \delta\left(L_{d, \alpha}-\mathcal{M}_{\alpha}\right) \\
& =\left(1+\frac{1}{2} \overline{\mathcal{B}}^{\alpha} \cdot \mathbf{b}+\overline{\mathcal{E}}^{\alpha} \cdot \mathbf{e}\right) Z_{\alpha} \delta\left(\omega-\epsilon_{\alpha}\right) .
\end{aligned}
$$

Note that the dual abelian electromagnetic fields are again defined to be on-shell as in $S U(2)$ case;

$$
\begin{aligned}
\overline{\mathcal{B}}_{j}^{\alpha} & \equiv i \epsilon_{j m l}\left(\partial_{k_{m}} \mathcal{A}_{l}^{\alpha}\right)_{\mid \omega=\epsilon_{\alpha}}, \overline{\mathcal{E}}_{j}^{\alpha} \equiv i\left(\partial_{\omega} \mathcal{A}_{j}^{\alpha}-\partial_{j} \mathcal{A}_{0}^{\alpha}\right)_{\mid \omega=\epsilon_{\alpha}}, \\
\mathcal{A}_{\mu}^{\alpha} & \equiv\left\langle u^{(0), \alpha} \mid \partial_{\mu} u^{(0), \alpha}\right\rangle, \\
\text { with } \mu & \mu \omega, k .
\end{aligned}
$$

In such a $U(1) \mathrm{FL}$, the time-reversal (spatial inversion) counterpart being already lifted at equilibrium, these linear responses against $\mathbf{b}$ and $\mathbf{e}$ can be measured only by the spectral weight itself for a given Fermi surface. Namely, the spin-filter are already implemented in matters themselves. Thereby, in stead of ARPES experiments, the resonating inelastic X-ray scattering (RIXS) experiment, which is by far compatible with applied electromagnetic fields, is a more promising photoemission experiment.

\section{V. $U(1)$ EFFECTIVE BOLTZMANN EQUATION AND EOM FOR QUASI-PARTICLES}

In the previous section, we clarified the physical implications of the first 4 terms of $\hat{\mathcal{L}}_{2}$ given in eq. (69). We will now study on quasi-particle dynamics, only to interpret remaining 4 terms in $\hat{\mathcal{L}}_{2}$ such as

$$
\begin{aligned}
\hat{\mathcal{L}}_{2}= & \cdots-\frac{i}{2}\left(\partial_{X_{k}} L_{d, \alpha}^{(0)}\right)\left[\hat{\Omega}_{Q_{k} X_{j}}^{\alpha},\left[\hat{D}_{Q_{j}}^{\alpha}, \hat{A}_{\alpha}\right]\right] \\
& -\left\{X_{j} \leftrightarrow Q_{j} \mid X_{k}, Q_{k}\right\}-\left\{X_{j}, Q_{j} \mid X_{k} \leftrightarrow Q_{k}\right\} \\
& +\left\{X_{j} \leftrightarrow Q_{j} \mid X_{k} \leftrightarrow Q_{k}\right\} .
\end{aligned}
$$

Strictly speaking, when it comes to an EOM for quasiparticles, we in principle have to begin with the Keldysh equation for the lesser (or greater) Green functions $\hat{\mathrm{g}}^{<(>)}$, i.e.

$$
\begin{aligned}
& {\left[G_{0}{ }^{-1}-\Sigma^{\mathrm{HF}}-\sigma, \mathrm{g}^{<(>)}\right]_{\otimes,-}-\left[\Sigma_{\mathrm{c}}^{<(>)}, \mathrm{b}\right]_{\otimes,-}} \\
& =\frac{1}{2}\left[\Sigma_{\mathrm{c}}^{>}, \mathrm{g}^{<}\right]_{\otimes,+}-\frac{1}{2}\left[\Sigma_{\mathrm{c}}^{<}, \mathrm{g}^{>}\right]_{\otimes,+}
\end{aligned}
$$

which is different from that for the spectral function $\hat{A}$.

As far as a system is not so far from its equilibration, however, this difference could be regarded as small at $T \simeq 0$ and $\omega \simeq \mu$. To be more specific, thanks to the boundary condition imposed on the Matsubara selfenergy and its continuity, both lesser and greater collisional self-energies $\hat{\Sigma}_{\mathrm{c}}^{<,>}(\omega)$ above become small around $\omega \simeq \mu$ (see the arguments in Appendix A2d and A3). As a result, we could begin with the following dissipationless equation, instead of eq. (91);

$$
\left[{\hat{G_{0}}}^{-1}-\hat{\Sigma}^{\mathrm{HF}}-\hat{\sigma}, \hat{\mathrm{g}}^{<(>)}\right]_{\otimes,-}=\hat{0} .
$$

Note that a small $\hat{\Sigma}_{\mathrm{c}}^{<,>}(\omega)_{\mid \omega \simeq \mu}$ does not necessarily lead to a small $\hat{\sigma}(\omega)_{\mid \omega \simeq \mu}$. Namely, the latter one is given by the energy- (principal) integral of the former two;

$$
\hat{\sigma}(\omega) \equiv \int_{-\infty}^{+\infty} \frac{d \omega^{\prime}}{2 \pi} \frac{\mathcal{P}}{\omega-\omega^{\prime}}\left(\hat{\Sigma}_{\mathbf{c}}^{<}\left(\omega^{\prime}\right)+\hat{\Sigma}_{\mathbf{c}}^{>}\left(\omega^{\prime}\right)\right) .
$$

With a help of this biased treatment of the collisional self-energies for different frequency regions, however, we can obtain clear physical interpretations for all the remaining terms in $\hat{\mathcal{L}}_{2}$ given in eq. (90). Namely, we can 
apply precisely a same projection process onto eq. (92) as we did for $\hat{A}$. Thus, we begin with the exactly same reduced Keldysh equation for $g_{\alpha}^{<}$as in eqs. (67,69), with $g_{\alpha}^{<}$being the $(\alpha, \alpha)$-th element of $N_{b}$ times $N_{b}$ matrix $\hat{g}^{<} \equiv \hat{U}^{\dagger} \hat{\mathrm{g}}^{<} \hat{U}$. By constructions, this diagonal element $g_{\alpha}^{<}$can be decomposed into the product between the generalized Fermi distribution function $f_{\alpha}$ (scalar quantity) and the spectral function for the $\alpha$-th band, later of which was already derived in the previous section ${ }^{34}$;

$$
g_{\alpha}^{<}(Q ; X)=A_{\alpha}(Q ; X) f_{\alpha}(Q ; X) .
$$

Generally speaking, based on this decomposition, one must derive a coupled effective Boltzmann equation for spin and charge, out of the $S U(2)$ RKEs for $g_{\alpha}^{<}$. However, since this analysis is somehow involved, we will henceforth restrict ourselves to $U(1) \mathrm{FLs}$, only to derive effective Boltzmann equation for charge degree of freedom, i.e. EOM only for $f_{\alpha}$.

In $U(1)$ case, substituting eq. (93) into the abelian RKE, we first obtain;

$$
\begin{aligned}
& 0=\left\{\mathcal{L}_{1}\left(A_{\alpha}\right)+\mathcal{L}_{2}\left(A_{\alpha}\right)\right\} \times f_{\alpha} \\
& +A_{\alpha} \times\left\{\partial_{X_{j}}\left(L_{d, \alpha}-\mathcal{M}_{\alpha}\right) \partial_{Q_{j}} f_{\alpha}\right. \\
& \left.-\partial_{X_{k}} \Omega_{Q_{k} X_{j}}^{\alpha} \partial_{Q_{j}} f_{\alpha}+\partial_{Q_{k}} \Omega_{X_{k} X_{j}}^{\alpha} \partial_{Q_{j}} f_{\alpha}-\left(X_{j} \leftrightarrow Q_{j}\right)\right\} .
\end{aligned}
$$

Notice that the spectral function $A_{\alpha}$ was determined such that $\mathcal{L}_{1}\left(A_{\alpha}\right)+\mathcal{L}_{2}\left(A_{\alpha}\right) \equiv 0$ (up to the 2 nd order in gradient expansion). Thus, in the right hand side, we can safely drop those terms which are proportional to $f_{\alpha}$ itself. Notice also that the spectral function $A_{\alpha}$ thus determined is sharply peaked at $\omega=\epsilon_{\alpha}$. Thereby, we have only to integrate this equation over frequency, such that $f_{\alpha}$ is replaced by the physical quasi-particle occupation number in the $q-R$ space;

$$
n_{\alpha}(q ; R, T) \equiv f_{\alpha}\left(q, \omega \equiv \epsilon_{\alpha} ; R, T\right) .
$$

The EOM thus obtained reads as follows;

$$
\begin{aligned}
& \left(1-\bar{\Omega}_{T \epsilon_{\alpha}}^{\alpha}+\left(\partial_{R_{j}} \epsilon_{\alpha}\right) \bar{\Omega}_{q_{j} \epsilon_{\alpha}}^{\alpha}-\left(\partial_{q_{j}} \epsilon_{\alpha}\right) \bar{\Omega}_{R_{j} \epsilon_{\alpha}}^{\alpha}\right) \partial_{T} n_{\alpha} \\
& =\left(\partial_{R_{j}} \epsilon_{\alpha}+\left(\partial_{T} \epsilon_{\alpha}\right) \bar{\Omega}_{\epsilon_{\alpha} R_{j}}^{\alpha}-\left(\partial_{R_{k}} \epsilon_{\alpha}\right) \bar{\Omega}_{q_{k} R_{j}}^{\alpha}+\bar{\Omega}_{T R_{j}}^{\alpha}\right. \\
& \left.\quad+\left(\partial_{q_{k}} \epsilon_{\alpha}\right) \bar{\Omega}_{R_{k} R_{j}}^{\alpha}\right) \partial_{q_{j}} n_{\alpha}-\left\{q_{j} \leftrightarrow R_{j}\right\},
\end{aligned}
$$

which we can regard as the Boltzmann equation for the $\alpha$-th band quasi-particles. Note that the partial $q, R$ and $T$-derivatives encoded into the curvatures therein apply only onto their explicit dependences and do not apply to their arguments of $\epsilon_{\alpha}$, e.g.

$$
\bar{\Omega}_{q_{i} R_{j}}^{\alpha} \equiv\left(\Omega_{q_{i} R_{j}}^{\alpha}\right)_{\mid \omega=\epsilon_{\alpha}}, \bar{\Omega}_{\epsilon_{\alpha} R_{j}}^{\alpha} \equiv\left(\Omega_{\epsilon_{\alpha} R_{j}}^{\alpha}\right)_{\mid \omega=\epsilon_{\alpha}} .
$$

As was explained in the introduction, however, one can also introduce the curvature defined in the codimensional space associated with $\omega \equiv \epsilon_{\alpha}(q, R, T)$, e.g.

$$
\begin{aligned}
\tilde{\Omega}_{q_{i} R_{j}}^{\alpha} & \equiv i\left(\partial_{q_{i}} \tilde{\mathcal{A}}_{R_{j}}^{\alpha}-\partial_{R_{j}} \tilde{\mathcal{A}}_{q_{i}}^{\alpha}\right), \\
\tilde{\mathcal{A}}_{R_{j}}^{\alpha} & \equiv\left\langle\tilde{u}^{\alpha} \mid \partial_{R_{j}} \tilde{u}^{\alpha}\right\rangle,\left|\tilde{u}^{\alpha}\right\rangle \equiv\left|u^{\alpha}\right\rangle_{\mid \omega=\epsilon_{\alpha}} .
\end{aligned}
$$

Then the Boltzmann equation above can be also expressed solely in terms of these curvatures in the $q-R-T$ space. Specifically, normalizing the coefficient of $\partial_{T} n_{\alpha}$, we obtain the following up to the 2 nd order in $\lambda$;

$$
\begin{aligned}
& 0=\partial_{T} n_{\alpha}+ \\
& +\left\{\partial_{R_{j}} \epsilon+\tilde{\Omega}_{T R_{j}}^{\alpha}-\left(\partial_{R_{k}} \epsilon\right) \tilde{\Omega}_{q_{k} R_{j}}^{\alpha}+\left(\partial_{q_{k}} \epsilon\right) \tilde{\Omega}_{R_{k} R_{j}}^{\alpha}\right\} \partial_{q_{j}} n_{\alpha} \\
& -\left\{\partial_{q_{j}} \epsilon+\tilde{\Omega}_{T q_{j}}^{\alpha}-\left(\partial_{R_{k}} \epsilon\right) \tilde{\Omega}_{q_{k} q_{j}}^{\alpha}+\left(\partial_{q_{k}} \epsilon\right) \tilde{\Omega}_{R_{k} q_{j}}^{\alpha}\right\} \partial_{R_{j}} n_{\alpha} \cdot(95)
\end{aligned}
$$

Accordingly, comparing this Boltzmann equation with the continuity equation, i.e. $0=\partial_{T} n_{\alpha}+\left(\partial_{T} q\right) \partial_{q} n_{\alpha}+$ $\left(\partial_{T} R\right) \partial_{R} n_{\alpha}$, one can readily read the effective EOM for the quasi-particle;

$$
\begin{gathered}
\frac{d R_{j}}{d T}=-\partial_{q_{j}} \epsilon-\tilde{\Omega}_{T q_{j}}^{\alpha}+\left(\partial_{R_{k}} \epsilon_{\alpha}\right) \tilde{\Omega}_{q_{k} q_{j}}^{\alpha}-\left(\partial_{q_{k}} \epsilon_{\alpha}\right) \tilde{\Omega}_{R_{k} q_{j}}^{\alpha}, \\
\frac{d q_{j}}{d T}=\partial_{R_{j}} \epsilon+\tilde{\Omega}_{T R_{j}}^{\alpha}-\left(\partial_{R_{k}} \epsilon_{\alpha}\right) \tilde{\Omega}_{q_{k} R_{j}}^{\alpha}+\left(\partial_{q_{k}} \epsilon_{\alpha}\right) \tilde{\Omega}_{R_{k} R_{j}}^{\alpha} .
\end{gathered}
$$

This effective EOM for quasi-particles proves, at least to the accuracy of 2 nd order in $\lambda$, that the EOM valid in "non-interacting" Fermi system ${ }^{\frac{5}{}}$ can be also generalized into "interacting" Fermi systems with those curvatures defined in the co-dimensional space, such as eq. (94). However, observing that the 1st order correction to the renormalization factor is characterized by the Berry's curvature in Euclidean $q-\omega-R-T$ space instead of that in co-dimensional space (see eq. (88)), studying the 3rd order correction to this EOM is still an interesting open question (see also sec. IV).

As an immediate application of this effective EOM, we can again consider the case with external electromagnetic fields;

$$
\begin{aligned}
& \frac{d R}{d T}=\mathbf{v}_{\alpha}+\tilde{\mathcal{B}}^{\alpha} \times \frac{d k}{d T}, \\
& \frac{d k}{d T}=-\mathbf{e}+\mathbf{b} \times \frac{d R}{d T} .
\end{aligned}
$$

where $\mathbf{v}_{\alpha} \equiv \partial_{k} \epsilon_{\alpha}$ and $\tilde{\mathcal{B}}^{\alpha}$ reads,

$$
\tilde{\mathcal{B}}^{\alpha} \equiv \overline{\mathcal{B}}^{\alpha}-\overline{\mathcal{E}}^{\alpha} \times \mathbf{v}_{\alpha} .
$$

Note that $\overline{\mathcal{B}}^{\alpha}$ and $\overline{\mathcal{E}}^{\alpha}$ are curvatures in Euclidean $k$ $\omega$ space (eqs.(213) and eqs.(1516) respectively), while $\tilde{\mathcal{B}}^{\alpha}$ is the curvature defined in the co-dimensional space (eqs. (910)). Observing eq. (96), one then see that the intrinsic AHE in $U(1)$ FLs should be defined in terms of both electromagnetic fields in the dual space;

$$
\sigma_{j m}=\epsilon_{j m l} \frac{e^{2}}{\hbar} \sum_{\alpha} \int \frac{d k}{(2 \pi)^{d}}\left(\overline{\mathcal{B}}^{\alpha}-\overline{\mathcal{E}}^{\alpha} \times \mathbf{v}_{\alpha}\right){ }_{l} n_{\mathrm{f}}\left(\epsilon_{\alpha, k}\right),
$$

where $n_{\mathrm{f}}$ denotes a Fermi distribution function.

Notice that the terminology "intrinsic" is now generalized into a slightly wider sense. Namely, the above expression for the Hall conductivity contains not only the " $k$-space magnetic field" effect, which is already present in a "non-interacting" limit, but also the "many-body 
effect" via the corresponding "electric field" component. Based on the Ishikawa-Matsuyama formula and Fermi liquid assumptions, one can also see that this "manybody" correction indeed takes over (a part of) the socalled vertex correction to the static transverse conductivity (see appendix B). This consistency check at the linear response regime strongly supports the validity of our derived effective EOM i.e. eqs.(96) and (97).

\section{SUMMARY AND OPEN PROBLEMS}

To extract an information of low-energy effective theory in a generic multiple-band interacting Fermi systems, we have derived the Reduced Keldysh Equation (RKE) which effectively describes the charge and spin degrees of freedom for a specific single-band forming a Fermi surface. Our derivation is perturbative with respect to the gradient expansion whose coupling constant measures how a system is disequilibrated. It is, however, nonperturbative in the electron-electron interactions. Instead, it relies only on the "adiabatic assumption" which is also utilized to validate the usual Fermi liquid framework. This assumption claims that, when it comes to the low-frequency region at sufficiently low temperature, the intrinsic life time of a quasi-particle (due to electronelectron interactions), which is $\mathcal{O}\left(T^{-2},(\omega-\mu)^{-2}\right)$, becomes much longer than the inverse of thermal broadening of the spectral functions $\left(\sim \mathcal{O}\left(T^{-1}\right)\right)$. This assumption usually provides a finite energy region within which Fermi liquid theory works. Based on the same spirit and as a sort of zero-th order approximation, we completely ignored the life-time part (anti-hermitian part) of the collisional self-energies, while studying on its hermitian part on a general ground.

Out of the RKE thus derived, we have succeeded in extracting several intriguing physical implications both in $S U(2)$ and $U(1)$ FLs. A first observation is that the linear response of the spectral weight for a quasi-particle with respect to an applied electromagnetic field, $\mathbf{e} / \mathbf{b}$, is characterized by (what we call) the dual electromagnetic fields, i.e. $\overline{\mathcal{E}}^{\alpha} / \overline{\mathcal{B}}^{\alpha}$. Since the linear response condition of small $\mathbf{e}$ and $\mathbf{b}$ precisely coincides with the condition of validity of the gradient expansion, these linear response expressions are asymptotically exact for any quasi-particle on a Fermi surface at zero temperature. Based on this theoretical observation, we also tried to give some rough idea of how to measure the $S U(2)$ Berry's curvatures in terms of photoemission experiments, so as to provide some future directions for the (spin) galvanomagnetic community (see sec. IV).

Another important achievement is our derivation of the effective equation of motion (EOM) for a quasi-particle in a $U(1) \mathrm{FL}$ up to the 2nd order accuracy in gradient expansion (see sec. V). From this equation, one sees that both the " $k$-space magnetic field" and its corresponding temporal component, dubbed as "electric field", contribute to the Lorentz force which acts on quasi-particles in $k$-space. In the linear response regime, this effective EOM is indeed consistent with the exact many-body formula for the static transverse conductivity (see appendix B).

We conclude with a discussion of future problems and issues. First, one ambiguity remains unresolved in our derivation of the reduced EOM. Specifically, working only to 2nd order in the gradient expansion, we cannot exclude the possibility that $\mathbf{v}_{\alpha}$ in eq. (98) may be more generally replaced by $\frac{d R}{d T}$. These two forms are equivalent up to 2nd order but not more generally. To fix this ambiguity, a further analysis including 3rd order effects in the gradient expansion would be necessary, which we leave for future work.

A more pressing issue is the well-controlled treatment of the anti-hermitian part of the collisional self-energies. Having completely ignored the corresponding terms by hand, our results are valid, in a strict sense, only for those quasi-particles exactly on a Fermi surface and at zero temperature. Since any physical quantities do involve non-zero excitation of quasiparticles (even in linear response), this is clearly not satisfactory. While one might naïvely expect that collisional effects can be included by direct analogy to classical kinetics (e.g. in the relaxation time or more sophisticated approximations), the possibility of some more interesting interplay between collisions and the Berry phase physics captured herein cannot be excluded. This may be of particular interest if one considers the instabilities of conventional metals toward ordered states such as superconductors, dielectrics, etc. A treatment of both the anti-hermitian part (decay of quasi-particles) and hermitian part (adiabatic transport of quasi-particles) of the RKE on an equal footing is certainly warranted in the future 22 .

\section{Acknowledgments}

The authors are pleased to acknowledge F. Duncan M. Haldane, Qian Niu, Congjun Wu, Jiangping Hu, Akio Kimura, Takayuki Kiss, Syunsuke Tsuda, Allan H. MacDonald and Akira Furusaki for their discussions and encouragements. The authors especially would like to thank Q. Niu for drawing our attention to the temporal component of $\hat{\mathcal{M}}_{\alpha}$, and to C.j. Wu for the discussion about the source of the dual electric fields. R. S. appreciated informations from A. Kimura, S. Tsuda and T. Kiss about photoemission experiments under external electromagnetic fields. This work was supported by NSF Grant DMR04-57440, PHY99-07949, and the Packard Foundation. R.S. was supported by JSPS Research Fellowships for Young Scientists. 


\section{APPENDIX A: BRIEF REVIEW OF KELDYSH EQUATION AND COLLISIONAL SELF-ENERGIES}

To make this paper self-contained, we will briefly review the derivation of the Keldysh equation ${ }^{25}$, defining several kinds of self-energies introduced in section II, such as $\hat{\Sigma}^{\mathrm{HF}}, \hat{\sigma}$ and $\hat{\Sigma}_{\mathrm{c}}^{<,>}$. In addition to this, we will also look into the boundary condition especially imposed on the collisional self-energy $\hat{\Sigma}_{\mathrm{c}}^{<,>}$. This boundary condition with additional arguments validates the dissipationless Keldysh equation for green functions, i.e. eqs. (92/A26), starting from which we derived the $U(1)$ effective Boltzmann equation in sec.V.

In the next subsection, we will begin with the timeordered Green function defined on the imaginary time domain $G\left(1,1^{\prime}\right)$ (see eq. (A1) for its definition). Specifically, the Dyson equation for this temperature (Matsubara) Green function is derived first. The Hartree-Fock and collisional self-energy encoded there are also iteratively defined in terms of this Matsubara Green function (see eqs. A5, A7) ).

The time-ordered Green function as a function of imaginary time $t$ is analytic separately in two region: $\operatorname{Im} t \in$ $[-\beta, 0]$ and $[0, \beta]$. In other words, we can introduce two functions, usually dubbed as lesser and greater Green functions, which coincide with this time-ordered one and which are analytic in these two regions respectively

$$
G\left(1,1^{\prime}\right)=\left\{\begin{array}{lll}
G^{>}\left(1,1^{\prime}\right) & \text { for } & \operatorname{Im} t_{1}<\operatorname{Im} t_{1^{\prime}} \\
-G^{<}\left(1,1^{\prime}\right) & \text { for } & \operatorname{Im} t_{1^{\prime}}<\operatorname{Im} t_{1}
\end{array}\right.
$$

(compare eq. (A1) with eqs. (A8|A9)). In the 2nd subsection of this appendix, keeping the analyticity of these two, we will extend its time domain from the imaginary time into the real time domain. Correspondingly, the Dyson equation is also analytically continued onto the real time domains (see eqs. A20|A22 A23).

We will see in the final subsection that periodic boundary condition imposed along the imaginary time axis relates the lesser and greater collisional self-energies defined on the real time domain with each other. (see eq. (A28)). This relation guarantees that, at zero temperature and at equilibrium, lesser and greater self-energies have no weight at $\omega<\mu$ and $\omega>\mu$ respectively, i.e. eqs. (A24/A25). These observations allow us to approximate the Keldysh equation derived further into the so-called dissipationless Keldysh equation (see the arguments from eq. (A22) to eq. (A26)).

\section{Dyson equation for temperature Green function}

Let us begin with the imaginary time Green function defined in the "interaction" representations:

$$
G\left(1,1^{\prime} ; \phi, t_{0}\right) \equiv \frac{1}{i} \frac{\left\langle\mathcal{T}\left\{\hat{S} \psi(1) \psi^{\dagger}\left(1^{\prime}\right)\right\}\right\rangle}{\langle\mathcal{T}\{\hat{S}\}\rangle}
$$

$$
\begin{aligned}
& =\frac{1}{i} \frac{\operatorname{Tr}\left[e^{-\beta(\mathcal{H}-\mu N)} \mathcal{T}\left\{\hat{S} \psi(1) \psi^{\dagger}\left(1^{\prime}\right)\right\}\right]}{\operatorname{Tr}\left[e^{-\beta(\mathcal{H}-\mu N)} \mathcal{T}\{\hat{S}\}\right]}, \\
\hat{S} & \equiv \exp \left[-i \int_{t_{0}}^{t_{0}-i \beta} d 2 \phi(2) \cdot \hat{n}(2)\right] \cdot(\mathrm{A} 1)
\end{aligned}
$$

Note that the argument of the fermion operator " $j$ " is an abbreviation of $\left(r_{j}, t_{j}, \alpha_{j}\right)$. Accordingly, $\int_{t_{0}}^{t_{0}-i \beta} d j$ includes not only the integral with respect to the imaginary time $t_{j}$, but also the summation over the band indices $\alpha_{j}$ and the integral over the spatial coordinate;

$$
\int_{t_{0}}^{t_{0}-i \beta} d j \equiv \sum_{\alpha_{j}} \int_{t_{0}}^{t_{0}-i \beta} d t_{j} \int d r_{j} .
$$

" $\mathcal{T}$ " is the imaginary-time-ordering operator along $\left[t_{0}, t_{0}-i \beta\right]$, where $t_{0}$ is always real-valued (In the next subsection, we will set $t_{0}$ to $-\infty$, after analytically continue $t_{1}$ and $t_{1}$, onto the real time domain.);

$\mathcal{T}\left\{\psi(1) \psi\left(1^{\prime}\right)\right\} \equiv \begin{cases}\psi(1) \psi\left(1^{\prime}\right) & \text { for } \operatorname{Im} t_{1}<\operatorname{Im} t_{1^{\prime}}, \\ -\psi\left(1^{\prime}\right) \psi(1) & \text { for } \operatorname{Im} t_{1^{\prime}}<\operatorname{Im} t_{1} .\end{cases}$

The time dependence of the fermion operator $\psi(1) \equiv$ $\psi_{\alpha_{1}}\left(r_{1}, t_{1}\right)$ is specified by $\mathcal{H} \equiv \mathcal{H}_{0}+\mathcal{H}_{1}$ :

$$
-i \partial_{t_{1}} \psi(1)=[\mathcal{H}, \psi(1)],
$$

with $\mathcal{H}_{0}$ and $\mathcal{H}_{1}$ given in eqs. (1112). Then taking the $t_{1}$-derivative of eq. (A1), we have the following EOM for this 1-point Green function;

$$
\begin{aligned}
& \int_{t_{0}}^{t_{0}-i \beta} d \overline{1} G_{0}^{-1}(1, \overline{1}) G\left(\overline{1}, 1^{\prime}\right)=\delta\left(1-1^{\prime}\right) \\
& \quad-i \int d 2 V(1,2) G_{2}\left(1,2,1^{\prime}, 2+\right)_{\left|t_{2}=t_{1}-i\right| \epsilon \mid},
\end{aligned}
$$

where the inverse of a bare Green function $G_{0}^{-1}\left(1,1^{\prime}\right)$ and two-point Green function are defined as follows;

$$
\begin{aligned}
G_{0}^{-1}\left(1,1^{\prime}\right) & \equiv\left[\left(i \partial_{t_{1}}-\phi(1)\right) \delta_{\alpha_{1} \alpha_{1^{\prime}}}-\hat{H}_{0}\right] \delta\left(1-1^{\prime}\right), \\
G_{2}\left(1,2,1^{\prime}, 2^{\prime}\right) & \equiv-\frac{\left\langle\mathcal{T}\left\{\hat{S} \psi(1) \psi(2) \psi^{\dagger}\left(2^{\prime}\right) \psi^{\dagger}\left(1^{\prime}\right)\right\}\right\rangle}{\langle\mathcal{T}\{\hat{S}\}\rangle} .
\end{aligned}
$$

" $2+$ " in eq. (A2) means that its temporal argument is chosen to be infinitesimally later than $t_{2}$ along the imaginary time axis; $2+\equiv\left(\alpha_{2}, r_{2}, t_{2}-i|\epsilon|\right)$.

The auxiliary scalar potential $\phi(2)$ entering into eq. (A1) as an "interaction" is utilized so as to describe the above 2-point Green function in terms of the selfenergy $\Sigma$ and 1-point Green's function $G$;

$$
\int_{t_{0}}^{t_{0}-i \beta} d \overline{1}\left[G_{0}^{-1}(1, \overline{1})-\Sigma(1, \overline{1})\right] G\left(\overline{1}, 1^{\prime}\right)=\delta\left(1-1^{\prime}\right)(\mathrm{A} 3)
$$

Notice first that the functional derivative of the 1-point Green function with respect to $\phi(2)$ brings about the 2point Green function;

$$
G_{2}\left(1,2,1^{\prime}, 2+\right)=\left[G(2,2+)-\frac{\delta}{\delta \phi(2)}\right] G\left(1,1^{\prime}\right) .
$$


Thus, substituting this into eq. A2 , we obtained a closed equation for the 1-point Green function;

$$
\begin{aligned}
& \int_{t_{0}}^{t_{0}-i \beta} d \overline{1} G_{0}^{-1}(1, \overline{1}) G\left(\overline{1}, 1^{\prime}\right)=\delta\left(1-1^{\prime}\right) \\
- & i \int d 2 V(1,2)\left[G(2,2+)-\frac{\delta}{\delta \phi(2)}\right]_{\left|t_{2}=t_{1}-i\right| \epsilon \mid} G\left(1,1^{\prime}\right) .(
\end{aligned}
$$

This self-consistent equation can be readily transcribed into that for the self-energy $\Sigma$. Matrix-multiplying eq. (A4) by $G^{-1} \equiv G_{0}^{-1}-\Sigma$, we first obtain the following;

$$
\begin{aligned}
& \Sigma\left(1,1^{\prime}\right)=-i \delta\left(1-1^{\prime}\right) \int d 2 V(1,2) G(2,2+)_{\left|t_{2}=t_{1}-i\right| \epsilon \mid} \\
& -i \int_{t_{0}}^{t_{0}-i \beta} d \overline{1} \int d 2 V(1,2) G(1, \overline{1}){\frac{\delta G^{-1}\left(\overline{1}, 1^{\prime}\right)}{\delta \phi(2)}}_{\left|t_{2}=t_{1}-i\right| \epsilon \mid} .
\end{aligned}
$$

Using $G^{-1}=G_{0}^{-1}-\Sigma$ and $\frac{\delta G_{0}^{-1}\left(1,1^{\prime}\right)}{\delta \phi(2)}=-\delta\left(1-1^{\prime}\right) \delta(1-$ 2 ) in the right hand side above, the following iterative equation for the self-energy $\Sigma$ is derived:

$$
\begin{aligned}
& \Sigma\left(1,1^{\prime}\right)=-i \delta\left(1-1^{\prime}\right) \int d 2 V(1,2) G(2,2+)_{\left|t_{2}=t_{1}-i\right| \epsilon \mid} \\
& \quad+i \delta\left(t_{1}-t_{1^{\prime}}\right) V\left(1,1^{\prime}\right) G\left(1,1^{\prime}\right)_{\left|t_{1^{\prime}}=t_{1}-i\right| \epsilon \mid} \\
& \quad+i \int_{t_{0}}^{t_{0}-i \beta} d \overline{1} \int d 2 V(1,2) G(1, \overline{1}){\frac{\delta \Sigma\left(\overline{1}, 1^{\prime}\right)}{\delta \phi(2)}}_{\left|t_{2}=t_{1}-i\right| \epsilon \mid}^{(\mathrm{A} 5)}
\end{aligned}
$$

Being proportional to $\delta\left(t_{1}-t_{1^{\prime}}\right)$, the first 2 terms are temporally instantaneous, which we usually call the Hartree-Fock term;

$$
\begin{aligned}
\Sigma^{\mathrm{HF}} \equiv- & i \delta\left(1-1^{\prime}\right) \int d 2 V(1,2) G(2,2+)_{\left|t_{2}=t_{1}-i\right| \epsilon \mid} \\
& +i \delta\left(t_{1}-t_{1^{\prime}}\right) V\left(1,1^{\prime}\right) G\left(1,1^{\prime}\right)_{\left|t_{1^{\prime}}=t_{1}-i\right| \epsilon \mid} \cdot(\mathrm{A} 6)
\end{aligned}
$$

A successive iteration through the 3rd term of eq. (A5 leads to the self-energy with higher order in $V$, which is clearly temporally non-instantaneous. We dub this noninstantaneous self-energy as the collisional self-energy henceforth;

$$
\Sigma_{\mathrm{c}}\left(1,1^{\prime}\right) \equiv \Sigma\left(1,1^{\prime}\right)-\Sigma^{\mathrm{HF}}\left(1,1^{\prime}\right) .
$$

Eq. (A3) in combination with eq. A5 is the Dyson equation for the imaginary-time 1-point Green function.

\section{Analytic continuations}

a. analytic continuation of $G^{<}$and $G^{>}$

Real-time Green functions are analytically continued from the following lesser and greater Green functions defined on the imaginary time domain;

$$
\begin{aligned}
G^{>}\left(1,1^{\prime} ; \phi, t_{0}\right) & =-i \frac{\left\langle\mathcal{U}\left(t_{0}, t_{0}-i \beta\right) \mathcal{U}^{-1}\left(t_{0}, t_{1}\right) \psi(1) \mathcal{U}\left(t_{0}, t_{1}\right) \mathcal{U}^{-1}\left(t_{0}, t_{1^{\prime}}\right) \psi^{\dagger}\left(1^{\prime}\right) \mathcal{U}\left(t_{0}, t_{1^{\prime}}\right)\right\rangle}{\left\langle\mathcal{U}\left(t_{0}, t_{0}-i \beta\right)\right\rangle} \\
G^{<}\left(1,1^{\prime} ; \phi, t_{0}\right) & =i \frac{\left\langle\mathcal{U}\left(t_{0}, t_{0}-i \beta\right) \mathcal{U}^{-1}\left(t_{0}, t_{1^{\prime}}\right) \psi^{\dagger}\left(1^{\prime}\right) \mathcal{U}\left(t_{0}, t_{1^{\prime}}\right) \mathcal{U}^{-1}\left(t_{0}, t_{1}\right) \psi(1) \mathcal{U}\left(t_{0}, t_{1}\right)\right\rangle}{\left\langle\mathcal{U}\left(t_{0}, t_{0}-i \beta\right)\right\rangle}
\end{aligned}
$$

$\mathcal{U}\left(t, t^{\prime}\right) \equiv \mathcal{T}\left\{\exp \left[-i \int_{t}^{t^{\prime}} d 1 \phi(1) \cdot \hat{n}(1)\right]\right\}$ characterizes the temporal evolution along the imaginary time axis $\left[t_{0}, t_{0}-\right.$ $i \beta]$ and thus is not unitary operator.

Note that, at equilibrium and with $\phi \equiv 0$, these two functions are analytic in $\operatorname{Im}\left(t_{1}-t_{1^{\prime}}\right) \in[-\beta, 0]$ and $[0, \beta]$ respectively, regardless of Re $t_{1}$ and Re $t_{1^{\prime}}$. Then, assuming that these analyticities also hold true in a weakly disequilibrated system with finite $\phi$, we will introduce separately the lesser and greater Green functions defined on the real time domain as follows;

$$
\left\{\begin{array}{l}
\mathrm{g}_{\alpha_{1} \alpha_{1^{\prime}}}^{<}\left(r_{1}, r_{1^{\prime}}: \operatorname{Re} t_{1}, \operatorname{Re} t_{1^{\prime}} ; \phi\right) \\
\equiv \lim _{t_{0} \rightarrow-\infty} G^{<}\left(1,1^{\prime} ; \phi, t_{0}\right) \mid \operatorname{Im} t_{1}=\operatorname{Im} t_{1^{\prime}+|\epsilon|=|\epsilon|}(\mathrm{A} 10) \\
\mathrm{g}_{\alpha_{1} \alpha_{1^{\prime}}}^{>}\left(r_{1}, r_{1^{\prime}}: \operatorname{Re} t_{1}, \operatorname{Re} t_{1^{\prime}} ; \phi\right) \\
\equiv \lim _{t_{0} \rightarrow-\infty} G^{>}\left(1,1^{\prime} ; \phi, t_{0}\right)\left|\operatorname{Im} t_{1}+\right| \epsilon \mid=\operatorname{Im} t_{1^{\prime}}=0
\end{array}\right.
$$

by taking $\epsilon$ infinitesimally small. Choose the test scalar field $\phi(1)$ such that it vanishes at Re $t_{1} \rightarrow-\infty$. Then, $\mathcal{U}\left(t_{0}, t_{0}-i \beta\right)$ appearing in both the denominator and the numerator of eqs. (A8A9 reduces to unit, when $t_{0} \rightarrow$ $-\infty$. Accordingly, the two functions in the right hand side are solely defined on the real domain;

$$
\begin{aligned}
& \mathrm{g}^{>}\left(1,1^{\prime} ; \phi\right)=-i\left\langle\psi_{\phi}(1) \psi_{\phi}^{\dagger}\left(1^{\prime}\right)\right\rangle \\
& \mathrm{g}^{<}\left(1,1^{\prime} ; \phi\right)=i\left\langle\psi_{\phi}^{\dagger}\left(1^{\prime}\right) \psi_{\phi}(1)\right\rangle,
\end{aligned}
$$

with $\psi_{\phi}(1) \equiv \mathcal{U}^{\dagger}\left(-\infty, t_{1}\right) \psi(1) \mathcal{U}\left(-\infty, t_{1}\right)$. Now that $\mathcal{U}(-\infty, t)$ is a unitary operator, we can construct a realtime-ordered Green function in terms of eqs. A11 A12);

$$
\begin{aligned}
\mathrm{g}\left(1,1^{\prime} ; \phi\right) & \equiv i\left\langle\mathcal{T}\left\{\psi_{\phi}(1) \psi_{\phi}\left(1^{\prime}\right)\right\}\right\rangle \\
& \equiv \begin{cases}\mathrm{g}^{>}\left(1,1^{\prime} ; \phi\right) & \text { for } t_{1}<t_{1^{\prime}} \\
-\mathrm{g}^{<}\left(1,1^{\prime} ; \phi\right) & \text { for } t_{1^{\prime}}<t_{1}\end{cases}
\end{aligned}
$$

$$
\text { b. analytic continuation of } \hat{\Sigma}_{\mathrm{c}}^{<} \text {and } \hat{\Sigma}_{\mathrm{c}}^{>}
$$

The Dyson equation being composed also of the selfenergy, let us next look into the analytic continuation 
of the collisional self-energy. The analytic property of the collisional self-energy can be obtained from that of Green functions at equilibrium. At equilibrium, both the 1-point Green function $G\left(1,1^{\prime}\right)$ and the 2-point Green function $G_{2}\left(1,2,1^{\prime}, 2+i|\epsilon|\right)\left|t_{2}=t_{1}-i\right| \epsilon \mid$ are analytic in two regions, i.e. $\operatorname{Im}\left(t_{1}-t_{1^{\prime}}\right) \in[-\beta, 0]$ and $[0, \beta]$, separately. Compare these analyticities with the relation among the self-energy, 1-point Green function and 2-point Green function;

$$
\begin{aligned}
& \int_{t_{0}}^{t_{0}-i \beta} d 2 V(1,2) G_{2}\left(1,2,1^{\prime}, 2+i|\epsilon|\right)\left|t_{2}=t_{1}-i\right| \epsilon \mid \\
& \equiv \int_{t_{0}}^{t_{0}-i \beta} d 2 \Sigma(1,2) \cdot G\left(2,1^{\prime}\right) .
\end{aligned}
$$

Then one can readily see that $\Sigma_{\mathrm{c}}\left(1,1^{\prime}\right)$ is also analytic both at these two regions, but separately. Thus, we can introduce the lesser and greater self-energy, such that they are analytic in the following two regions respectively;

$$
\Sigma_{\mathrm{c}}\left(1,1^{\prime}\right) \equiv \begin{cases}\Sigma_{\mathrm{c}}^{>}\left(1,1^{\prime}\right) & \text { for } \operatorname{Im} t_{1}<\operatorname{Im} t_{1^{\prime}} \\ -\Sigma_{\mathrm{c}}^{<}\left(1,1^{\prime}\right) & \text { for } \operatorname{Im} t_{1^{\prime}}<\operatorname{Im} t_{1} .\end{cases}
$$

Exactly in a same way as we did for the 1-point Green function, we can then formally extend these two selfenergies onto the real time domain separately ;

$$
\left\{\begin{array}{l}
\Sigma_{\mathrm{c} \alpha_{1}, \alpha_{1^{\prime}}}^{<}\left(r_{1}, r_{1^{\prime}}: \operatorname{Re} t_{1}, \operatorname{Re} t_{1^{\prime}} ; \phi\right) \\
\equiv \lim _{t_{0} \rightarrow-\infty} \Sigma_{\mathrm{c}}^{<}\left(1,1^{\prime} ; \phi, t_{0}\right) \mid \operatorname{Im} t_{1}=\operatorname{Im} t_{1^{\prime}+|\epsilon|=|\epsilon|}( \\
\Sigma_{\mathrm{c} \alpha_{1}, \alpha_{1^{\prime}}}^{>}\left(r_{1}, r_{1^{\prime}}: \operatorname{Re} t_{1}, \operatorname{Re} t_{1^{\prime}} ; \phi\right) \\
\equiv \lim _{t_{0} \rightarrow-\infty} \Sigma_{\mathrm{c}}^{>}\left(1,1^{\prime} ; \phi, t_{0}\right)\left|\operatorname{Im} t_{1}+\right| \epsilon \mid=\operatorname{Im} t_{1^{\prime}}=0
\end{array}\right.
$$

\section{c. analytic continuation of Dyson equation}

Now that both the self-energy and Green functions are analytically continued onto the real-time domain, we will derive the Dyson equation for these real-time functions. We begin with that for the imaginary time domain;

$$
\begin{aligned}
& \left(\hat{G}_{0}^{-1}-\hat{\Sigma}^{\mathrm{HF}}\right) \cdot G^{<}\left(1,1^{\prime}\right) \\
& =\int_{t_{0}}^{t_{0}-i \beta} d \overline{1} \Sigma_{\mathrm{c}}(1, \overline{1}) G\left(\overline{1}, 1^{\prime}\right) .
\end{aligned}
$$

Decompose first the right hand side into three terms, such that each term is expressed solely in terms of lesser / greater Green function / collisional self-energy. Namely, depending on whether $t_{\overline{1}}$ locates within $\left[t_{0}, t_{1}\right],\left[t_{1}, t_{1^{\prime}}\right]$ or $\left[t_{1^{\prime}}, t_{0}-i \beta\right]$, the right hand side can be divided into the following three parts;

$$
\begin{aligned}
& \left(\hat{G}_{0}^{-1}-\hat{\Sigma}^{\mathrm{HF}}\right) \cdot G^{<}\left(1,1^{\prime}\right) \\
& =\int_{t_{0}}^{t_{1}} d \overline{1} \Sigma_{\mathrm{c}}^{>}(1, \overline{1}) G^{<}\left(\overline{1}, 1^{\prime}\right)+\int_{t_{1}}^{t_{1^{\prime}}} d \overline{1} \Sigma_{\mathrm{c}}^{<}(1, \overline{1}) G^{<}\left(\overline{1}, 1^{\prime}\right) \\
& \quad+\int_{t_{1^{\prime}}}^{t_{0}-i \beta} d \overline{1} \Sigma_{\mathrm{c}}^{<}(1, \overline{1}) G^{>}\left(\overline{1}, 1^{\prime}\right),
\end{aligned}
$$

where the integral path is still along $\left[t_{0}, t_{0}-i \beta\right]$ (see Fig. 5(a)). Then, we will deform this integral path so that both $t_{1}$ and $t_{1}$, reach the real-time axis. During this deformation, however, to keep the analyticity for all the Green functions and self-energies in the both right and left hand sides, we have to observe the following condition;

$$
-\beta<\operatorname{Im} t_{1^{\prime}}<\operatorname{Im} t_{1}<0
$$

Due to this condition, for given "target" Re $t_{1}$ and Re $t_{1^{\prime}}$, our integral path is uniquely deformed into a "L" shaped path depicted in Fig. 5 (b-c);

$$
\begin{aligned}
& \left(\hat{G}_{0}^{-1}-\hat{\Sigma}^{\mathrm{HF}}\right) \cdot G^{<}\left(1,1^{\prime}\right) \\
& =\int_{t_{0}}^{t_{1}} d \overline{1} \Sigma_{\mathrm{c}}^{>}(1, \overline{1}) G^{<}\left(\overline{1}, 1^{\prime}\right)+\int_{t_{1}}^{t_{1^{\prime}}} d \overline{1} \Sigma_{\mathrm{c}}^{<}(1, \overline{1}) G^{<}\left(\overline{1}, 1^{\prime}\right)+ \\
& \int_{t_{1^{\prime}}}^{t_{0}-i 0} d \overline{1} \Sigma_{\mathrm{c}}^{<}(1, \overline{1}) G^{>}\left(\overline{1}, 1^{\prime}\right)+\int_{t_{0}-i 0}^{t_{0}-i \beta} d \overline{1} \Sigma_{\mathrm{c}}^{<}(1, \overline{1}) G^{>}\left(\overline{1}, 1^{\prime}\right) .
\end{aligned}
$$

Note that the 1st three integral paths in the right hand side are all along the real time domain, while the last one is strictly along $\left[t_{0}, t_{0}-i \beta\right]$. So as to eliminate the final term, we will take $t_{0}$ to be $-\infty$. In this limit, $G^{>}\left(\overline{1}, 1^{\prime} ; \phi\right)$ in the last term should vanish, since $\left|\operatorname{Re} t_{\overline{1}}-\operatorname{Re} t_{1^{\prime}}\right| \rightarrow \infty$. This procedure simultaneously completes the analytic continuations of all the Green functions / collisional selfenergies encoded in this equation (see eqs. (A10 A15) );

$$
\begin{aligned}
& \left(\hat{G}_{0}^{-1}-\hat{\Sigma}^{\mathrm{HF}}\right) \cdot \mathrm{g}^{<}\left(1,1^{\prime}\right) \\
& =\int_{-\infty}^{t_{1}} d \overline{1} \Sigma_{\mathrm{c}}^{>}(1, \overline{1}) \mathrm{g}^{<}\left(\overline{1}, 1^{\prime}\right)+\int_{t_{1}}^{t_{1^{\prime}}} d \overline{1} \Sigma_{\mathrm{c}}^{<}(1, \overline{1}) \mathrm{g}^{<}\left(\overline{1}, 1^{\prime}\right) \\
& \quad+\int_{t_{1^{\prime}}}^{-\infty} d \overline{1} \Sigma_{\mathrm{c}}^{<}(1, \overline{1}) \mathrm{g}^{>}\left(\overline{1}, 1^{\prime}\right) \\
& =\int_{-\infty}^{t_{1}} d \overline{1}\left[\Sigma_{\mathrm{c}}^{>}(1, \overline{1})-\Sigma_{\mathrm{c}}^{<}(1, \overline{1})\right] \mathrm{g}^{<}(\overline{1}, 1) \\
& \quad-\int_{-\infty}^{t_{1^{\prime}}} d \overline{1} \Sigma_{\mathrm{c}}^{<}(1, \overline{1})\left[\mathrm{g}^{>}\left(\overline{1}, 1^{\prime}\right)-\mathrm{g}^{<}\left(\overline{1}, 1^{\prime}\right)\right] .
\end{aligned}
$$

In a similar way, we can easily obtain an equation of motion for the greater Green function;

$$
\begin{aligned}
& \left(\hat{G}_{0}^{-1}-\hat{\Sigma}^{\mathrm{HF}}\right) \mathrm{g}^{>}\left(1,1^{\prime}\right) \\
& =\int_{-\infty}^{t_{1}} d \overline{1}\left[\Sigma_{\mathrm{c}}^{>}(1, \overline{1})-\Sigma_{\mathrm{c}}^{<}(1, \overline{1})\right] \mathrm{g}^{>}(\overline{1}, 1) \\
& \quad-\int_{-\infty}^{t_{1^{\prime}}} d \overline{1} \Sigma_{\mathrm{c}}^{>}(1, \overline{1})\left[\mathrm{g}^{>}\left(\overline{1}, 1^{\prime}\right)-\mathrm{g}^{<}\left(\overline{1}, 1^{\prime}\right)\right] .
\end{aligned}
$$

At equilibrium, both Green function and self-energy become translationally invariant in space and time, e.g.

$$
\begin{aligned}
\mathrm{g}^{<(>)}\left(1,1^{\prime} ; \phi \equiv 0\right) & =\mathrm{g}_{\alpha_{1}, \alpha_{1^{\prime}}}^{<(>)}\left(r_{1}-r_{1^{\prime}}, t_{1}-t_{1^{\prime}}\right) \\
\Sigma_{\mathrm{c}}^{<(>)}\left(1,1^{\prime} ; \phi \equiv 0\right) & =\Sigma_{\mathrm{c}}^{<(>)}{ }_{\alpha_{1}, \alpha_{1^{\prime}}}\left(r_{1}-r_{1^{\prime}}, t_{1}-t_{1^{\prime}}\right) .
\end{aligned}
$$




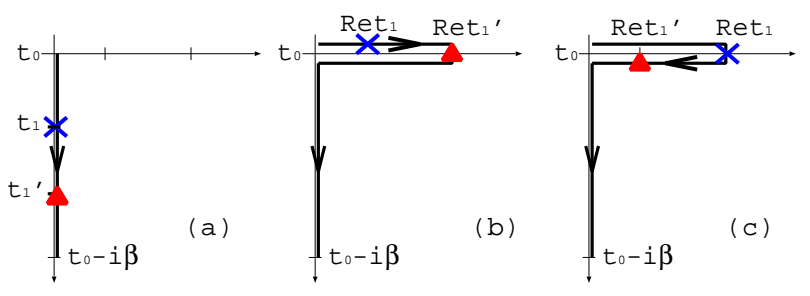

FIG. 5: (a): The integral path for eq. A16). (b): Integral path for eq. (A18) in the case of $\operatorname{Re} t_{1}<\operatorname{Re} t_{1}{ }^{\prime}$. (c) That for $\operatorname{Re} t_{1}>\operatorname{Re} t_{1}{ }^{\prime}$.

Thus, when Fourier-transformed with respect to these relative coordinates, the convolutions appearing in eq. A20 would have reduced to a simple product, if the associated integral region were to range $[-\infty, \infty]$. In reality, however, their temporal integral regions do not range from $-\infty$ to $+\infty$. As a result, when Fourier transformed, they end up with several principal integrals with respect to the frequency. The hermitian part of the collisional self-energy and Green function solely take over these principal integral parts. To be specific, introduce the following two functions;

$$
\begin{aligned}
\mathrm{b}\left(1,1^{\prime}\right) & \equiv \frac{1}{2} \frac{t_{1}-t_{1^{\prime}}}{\left|t_{1}-t_{1^{\prime}}\right|}\left[\mathrm{g}^{>}\left(1,1^{\prime}\right)-\mathrm{g}^{<}\left(1,1^{\prime}\right)\right], \\
\sigma\left(1,1^{\prime}\right) & \equiv \frac{1}{2} \frac{t_{1}-t_{1^{\prime}}}{\left|t_{1}-t_{1^{\prime}}\right|}\left[\Sigma_{\mathrm{c}}^{>}\left(1,1^{\prime}\right)-\Sigma_{\mathrm{c}}^{<}\left(1,1^{\prime}\right)\right] .(\mathrm{A} 21)
\end{aligned}
$$

Then we can formally rewrite the above equation in such a way that their convolutions with respect to time are always taken over $[-\infty, \infty]$;

$$
\begin{aligned}
\left(\hat{G}_{0}^{-1}-\hat{\Sigma}^{\mathrm{HF}}\right) \otimes \mathrm{g}^{<}= & \sigma \otimes \mathrm{g}^{<}+\Sigma_{\mathrm{c}}^{<} \otimes \mathrm{b} \\
& +\frac{1}{2}\left(\Sigma_{\mathrm{c}}^{>} \otimes \mathrm{g}^{<}-\Sigma_{\mathrm{c}}^{<} \otimes \mathrm{g}^{>}\right), \\
\left(\hat{G}_{0}^{-1}-\hat{\Sigma}^{\mathrm{HF}}\right) \otimes \mathrm{g}^{>}= & \sigma \otimes \mathrm{g}^{>}+\Sigma_{\mathrm{c}}^{>} \otimes \mathrm{b} \\
& +\frac{1}{2}\left(\Sigma_{\mathrm{c}}^{>} \otimes \mathrm{g}^{<}-\Sigma_{\mathrm{c}}^{<} \otimes \mathrm{g}^{>}\right) .
\end{aligned}
$$

where we used the following abbreviated notations;

$$
(A \otimes B)\left(1,1^{\prime}\right) \equiv \int_{-\infty}^{\infty} d \overline{1} A(1, \overline{1}) B\left(\overline{1}, 1^{\prime}\right) .
$$

\section{d. dissipationless Keldysh equation}

Eqs. A22 A23 are what we call the Keldysh equation, from which we readily obtain eq. $(13)^{32}$. Notice that the re-expression in terms of $\sigma$ and $\mathrm{b}$ is not just for its formality, but is in fact an important step to approximate this Keldysh equation, based on physical arguments. As we see in the next subsection, at zero temperature and at equilibrium, the periodic boundary condition along the imaginary time domain ensures that, when Fourier-transformed with respect to their relative coordinates, the lesser/greater collisional self-energy vanishes for those $\omega$ greater/less than chemical potential $\mu$ respectively;

$$
\begin{aligned}
& \hat{\Sigma}_{\mathrm{c}}^{<}(q, \omega)=\hat{0} \text { for } \omega>\mu, \\
& \hat{\Sigma}_{\mathrm{c}}^{>}(q, \omega)=\hat{0} \text { for } \omega<\mu .
\end{aligned}
$$

Thereby, provided that a system is weakly disturbed from this equilibrated situation, we could still assume that these relations hold true for the collisional self-energy, with $\mu$ generalized into a function depending on $R$ and $T ; \mu \rightarrow \mu(R, T)$. Observing further the continuity of these collisional self-energies around $\omega \simeq \mu$, we could regard both of them to be sufficiently small quantities at these low frequency regions, i.e. $\omega \simeq \mu$.

The hermitian part of the self-energy $\hat{\sigma}(\omega)$, however, is not necessarily a small quantity, even for $\omega \simeq \mu$. Namely, when Wigner-transformed, those lesser/greater self-energies for high frequency regions do contribute to $\hat{\sigma}(\omega)$ at the low frequency region ${ }^{34}$;

$$
\begin{aligned}
& \hat{\sigma}(q, R ; \omega, T)= \\
& \quad \int_{-\infty}^{+\infty} \frac{d \omega^{\prime}}{2 \pi} \frac{\mathcal{P}}{\omega-\omega^{\prime}}\left(\hat{\Sigma}_{\mathrm{c}}^{<}+\hat{\Sigma}_{\mathrm{c}}^{>}\right)\left(q, R ; \omega^{\prime}, T\right) .
\end{aligned}
$$

Due to this different behavior between $\sigma(\omega)$ and $\Sigma_{c}^{<(>)}(\omega)$ around $\omega \simeq \mu$, we can approximate eqs. (A22A23) into the following dissipationless Keldysh equations;

$$
\left(\hat{G}_{0}^{-1}-\hat{\Sigma}^{\mathrm{HF}}-\hat{\sigma}\right) \otimes \hat{\mathrm{g}}^{<(>)}=0 .
$$

which is supposed to be still valid for $\omega \simeq \mu$ at sufficiently low temperature.

\section{3. periodic boundary conditions}

Observing eq. (A14), we will first read the boundary condition (b.c.) for the collisional self-energy, out of those for the 1-point and 2-point Green functions. Notice that the 1-point Matsubara Green functions defined in eqs. A8 A9 obey the following boundary condition at equilibrium;

$$
G^{<}\left(1,1^{\prime}\right)=-e^{\beta \mu} G^{>}\left(1-i \beta, 1^{\prime}\right)
$$

for $-\beta<\operatorname{Im} t_{1^{\prime}}<\operatorname{Im} t_{1}<0$ and arbitrary Re $t_{1}$ and Re $t_{1^{\prime}}$. In a similar way, we can also see from its definition that the 2-point Matsubara Green function observes,

$$
\begin{aligned}
& G_{2}\left(1,2,1^{\prime}, 2+i|\epsilon|\right)_{\left|t_{2}=t_{1}-i\right| \epsilon \mid} \\
& \quad=-e^{\beta \mu} G_{2}\left(1-i \beta, 2,1^{\prime}, 2+i|\epsilon|\right)_{\left|t_{2}=t_{1}-i \beta-i\right| \epsilon \mid}
\end{aligned}
$$

for $-\beta<\operatorname{Im} t_{1^{\prime}}<\operatorname{Im} t_{1}<0$ and arbitrary Re $t_{1}$ and $\operatorname{Re} t_{1^{\prime}}$. When compared with eq. (A14), these two then lead to the following boundary condition for the collisional self-energy;

$$
\Sigma_{c}\left(1,1^{\prime}\right)=-e^{\beta \mu} \Sigma_{c}\left(1-i \beta, 1^{\prime}\right) .
$$


While being imposed on the imaginary time direction, this equation becomes useful, when Fourier-transformed with respect to $\operatorname{Re} t_{1}-\operatorname{Re}_{1}, \underline{34}$;

$$
\Sigma_{\mathrm{c}}^{<}\left(r_{1}, r_{1^{\prime}} ; \omega\right)=e^{-\beta(\omega-\mu)} \Sigma_{\mathrm{c}}^{>}\left(r_{1}, r_{1^{\prime}} ; \omega\right) .
$$

Namely, eq. A28) requires both of them to strictly observe eqs. A24A25) at zero temperature, since either $\Sigma_{\mathrm{c}}^{<}$or $\Sigma_{\mathrm{c}}^{>}$can not be singular in a usual metal.

\section{APPENDIX B: CONSISTENCY WITH ISHIKAWA-MATSUYAMA-HALDANE FORMULA}

The conductivities being given by the current-current correlation functions, they are usually subject under the vertex corrections in interacting Fermi systems. However, when it comes to the static and transverse component, the Ward identity relates this vertex part with the derivative of the 1-point Green function, such that the Hall conductivity is expressed solely in terms of the single-particle Green function;

$$
\sigma_{\lambda}=\frac{e^{2}}{2 \hbar} \frac{\epsilon_{\lambda \mu \nu}}{(2 \pi)^{d}} \int d k \int \frac{d \omega}{2 \pi} e^{i \omega 0+} \operatorname{Tr}\left[\frac{\partial \hat{g}}{\partial \omega} \frac{\partial \hat{g}^{-1}}{\partial k_{\mu}} \hat{g} \frac{\partial \hat{g}^{-1}}{\partial k_{\nu}}\right](\mathrm{B} 1)
$$

Specifically, let us choose the basis in eq. (B1), such that the Green function is diagonalized;

which is known as the Ishikawa-Matsuyama formula 21,31 . In this appendix, we will show that our derived expression for the $U(1)$ Hall conductivity, i.e. eq. (99), is in indeed consistent with this many-body formula, using only Fermi liquid assumptions. Namely, we will assume that, when diagonalized, each eigenvalue for the time-ordered 1-point Green function has a pole sufficiently closed to the real-axis, such that the corresponding quasi-particle life time is infinitely long;

$$
\begin{aligned}
{\left[\hat{g}_{d}\right]_{\alpha \alpha} } & \equiv\left[\hat{U}^{-1} \hat{g} \hat{U}\right]_{\alpha \alpha} \\
\quad= & \frac{1}{\omega-E_{\alpha, k}(\omega)-i \operatorname{sign}(\omega-\mu) \cdot 0+} .
\end{aligned}
$$

We can identify $\hat{U}$ above as the unitary matrix diagonalizing our Lagrangian $\hat{L}$.

$$
\begin{aligned}
\sigma_{\lambda}=\frac{e^{2}}{2 \hbar} \frac{\epsilon_{\lambda \mu \nu}}{(2 \pi)^{d}} \int d k \int \frac{d \omega}{2 \pi} e^{i \omega 0+} & \operatorname{Tr}\left[\left\{\left(\partial_{\omega} \hat{U}\right) \hat{g}_{d} \hat{U}^{-1}+\hat{U}\left(\partial_{\omega} \hat{g}_{d}\right) \hat{U}^{-1}+\hat{U} \hat{g}_{d}\left(\partial_{\omega} \hat{U}^{-1}\right)\right\}\right. \\
& \left\{\left(\partial_{k_{\mu}} \hat{U}\right) \hat{g}_{d}^{-1} \hat{U}^{-1}+\hat{U}\left(\partial_{k_{\mu}} \hat{g}_{d}^{-1}\right) \hat{U}^{-1}+\hat{U} \hat{g}_{d}^{-1}\left(\partial_{k_{\mu}} \hat{U}\right)^{-1}\right\} \\
& \left.\hat{U} \hat{g}_{d} \hat{U}^{-1}\left\{\left(\partial_{k_{\nu}} \hat{U}\right) \hat{g}_{d}^{-1} \hat{U}^{-1}+\hat{U}\left(\partial_{k_{\nu}} \hat{g}_{d}^{-1}\right) \hat{U}^{-1}+\hat{U} \hat{g}_{d}^{-1}\left(\partial_{k_{\nu}} \hat{U}^{-1}\right)\right\}\right]
\end{aligned}
$$

These 27 terms can be classified into 3 types, according to the matrix structure within the trace. To see this, notice first that, irrespective of being differentiated or not, $\hat{g}_{d}$ and $\hat{g}_{d}^{-1}$ alternate each other within the trace. When we have either $\hat{U}^{-1}\left(\partial_{Q} \hat{U}\right) \equiv \mathcal{A}_{Q}$ or $\left(\partial_{Q} \hat{U}^{-1}\right) \cdot\left(\partial_{Q^{\prime}} \hat{U}\right)$ between a pair of neighboring $\hat{g}_{d}$ and $\hat{g}_{d}^{-1}$, this pair of green function and its inverse can not be directly-connected. On the one hand, when both $\hat{U}$ and $\hat{U}^{-1}$ between a pair of $\hat{g}_{d}$ and $\hat{g}_{d}^{-1}$ are free from the derivative, these two can be clearly directly-connected. We will first classify all the terms appearing within the above integrand in terms of the number of directly-connected pairs of $\hat{g}_{d}$ and $\hat{g}_{d}^{-1}$.

One class is those terms having no pair of directly connected Green function and its inverse. For example, the following term belongs to this class;

$$
\begin{aligned}
& \operatorname{Tr}\left[\hat{U}\left(\partial_{\omega} \hat{g}_{d}\right) \hat{U}^{-1} \hat{U} \hat{g}_{d}^{-1}\left(\partial_{k_{\mu}} \hat{U}^{-1}\right) \hat{U} \hat{g}_{d} \hat{U}^{-1}\left(\partial_{k_{\nu}} \hat{U}\right) \hat{g}_{d}^{-1} \hat{U}^{-1}\right] \\
& \quad=\operatorname{Tr}\left[\left(\partial_{\omega} \hat{g}_{d}^{-1}\right) \hat{\mathcal{A}}_{k_{\mu}} \hat{g}_{d} \hat{\mathcal{A}}_{k_{\nu}}\right]
\end{aligned}
$$

where one pair of $\hat{g}_{d}$ and $\hat{g}_{d}^{-1}$ was directly-connected only to reduce into a unit, i.e. $\hat{g}_{d} \cdot \hat{g}_{d}^{-1}=1$. Such an anni- hilated pair is not regarded as a directly-connected pair. Among 27 terms enumerated above, there exist 16 terms belonging to this class, all of which can be summarized into several total derivatives;

$$
\begin{aligned}
& \partial_{k_{\nu}}\left\{\operatorname{Tr}\left[\hat{\mathcal{A}}_{\omega} \hat{g}_{d} \hat{\mathcal{A}}_{k_{\mu}} \hat{g}_{d}^{-1}\right]\right\} \\
& +\partial_{k_{\mu}}\left\{\operatorname{Tr}\left[\begin{array}{llll}
\hat{\mathcal{A}}_{k_{\nu}} & \hat{g}_{d} & \hat{\mathcal{A}}_{\omega} & \hat{g}_{d}^{-1}
\end{array}\right]\right\} \\
& \quad+\partial_{\omega}\left\{\operatorname{Tr}\left[\begin{array}{llll}
\hat{\mathcal{A}}_{k_{\nu}} & \hat{g}_{d} & \hat{\mathcal{A}}_{k_{\mu}} & \hat{g}_{d}^{-1}
\end{array}\right]\right\} \rightarrow 0 .
\end{aligned}
$$

Being contour-integrated, all these terms vanish as indicated.

The 2nd class is those terms having two directly connected pairs. Among 27 terms enumerated above, there clearly exists only one such a term;

$$
\begin{aligned}
& \operatorname{Tr}\left[\left(\partial_{\omega} \hat{g}_{d}\right)\left(\partial_{k_{\mu}} \hat{g}_{d}^{-1}\right) \hat{g}_{d}\left(\partial_{k_{\nu}} \hat{g}_{d}^{-1}\right)\right] \\
& \quad=\operatorname{Tr}\left[\left(\partial_{\omega} \hat{g}_{d}\right)\left(\partial_{k_{\nu}} \hat{g}_{d}^{-1}\right) \hat{g}_{d}\left(\partial_{k_{\mu}} \hat{g}_{d}^{-1}\right)\right],
\end{aligned}
$$

Then, observing the overall factor $\epsilon_{\lambda \mu \nu}$ in eq. (B2), we can readily drop this term. 
The 3rd class consists of 10 terms having only one directly connected pair. 4 terms being canceled by one another, we have the following 6 terms remained;

$$
\begin{aligned}
& -2 \operatorname{Tr}\left[\hat{g}_{d}^{-1}\left(\partial_{\omega} \hat{g}_{d}\right)\left(\partial_{k_{\mu}} \hat{U}^{-1}\right)\left(\partial_{k_{\nu}} \hat{U}\right)\right] \\
& -2 \operatorname{Tr}\left[\hat{g}_{d}^{-1}\left(\partial_{k_{\nu}} \hat{g}_{d}\right)\left(\partial_{\omega} \hat{U}^{-1}\right)\left(\partial_{k_{\mu}} \hat{U}\right)\right] \\
& \quad+2 \operatorname{Tr}\left[\hat{g}_{d}^{-1}\left(\partial_{k_{\nu}} \hat{g}_{d}\right)\left(\partial_{k_{\mu}} \hat{U}^{-1}\right)\left(\partial_{\omega} \hat{U}\right)\right] .
\end{aligned}
$$

Notice that, when either the band index $\alpha$ or its momentum $k$ denotes a filled Bloch state, the diagonalized time-ordered Green functions has a pole at the upper half plane in the complex $\omega$-plane, say, $\omega=\epsilon_{\alpha, k}+i 0+$;

$$
\left[\hat{g}_{d}^{-1}\left(\partial_{k_{\mu}} \hat{g}_{d}\right)\right]_{\alpha \alpha} \equiv \frac{\left(\partial_{k_{\mu}} \epsilon_{\alpha, k}\right)}{\omega-\epsilon_{\alpha, k}-i 0+}
$$

with $\epsilon_{\alpha, k}-E_{\alpha, k}\left(\epsilon_{\alpha, k}\right)=0$. Note that, in the right hand side, we have assumed the infinite life-time for these Bloch states. This assumption corresponds to ignoring the anti-Hermitian part of the collisional self-energy, i.e. $\hat{\Gamma}$.

Substituting eq. (B3) into eq. (B2), we then integrate over the frequency, such that the summation/integral regions over $\alpha / k$ are restricted within the filled Bloch states. Using further eq. (B4), we then observe that the Hall conductivity is indeed characterized by the dual version of $U(1)$ electromagnetic fields introduced in the text;

$$
\begin{aligned}
\sigma_{\lambda} & =\frac{e^{2}}{\hbar} \frac{1}{(2 \pi)^{d}} \sum_{\alpha} \int d k\left\{\overline{\mathcal{B}}^{\alpha}-\left(\overline{\mathcal{E}}^{\alpha} \times \mathbf{v}_{\alpha}\right)\right\}_{\lambda}, \\
\overline{\mathcal{B}}_{\lambda}^{\alpha} & =i \epsilon_{\lambda \mu \nu}\left\{\left[\left(\partial_{k_{\mu}} \hat{U}\right)^{\dagger}\left(\partial_{k_{\mu}} \hat{U}\right)\right]_{\alpha \alpha}\right\}_{\mid \omega=\epsilon_{\alpha, k}} \\
\overline{\mathcal{E}}_{\lambda}^{\alpha} & =i\left\{\left[\left(\partial_{\omega} \hat{U}\right)^{\dagger}\left(\partial_{k_{\lambda}} \hat{U}\right)-\text { c.c. }\right]_{\alpha \alpha}\right\}_{\mid \omega=\epsilon_{\alpha, k}},
\end{aligned}
$$

with $\mathbf{v}_{\alpha, \lambda}=\left(\partial_{k_{\lambda}} \epsilon_{\alpha, k}\right)$.

\section{APPENDIX C: AMPERE'S LAW}

In a non-interacting Fermi system, the Gauss law solely determines the distribution of the $U(1)$ magnetic field. When a doubly degeneracy point is formed by two neighboring energy dispersions, say $\alpha$-th and $(\alpha+1)$-th band at $k=k^{\mathrm{mm}}$,

$$
L_{d, \alpha}\left(k^{\mathrm{mm}}\right)=L_{d, \alpha+1}\left(k^{\mathrm{mm}}\right),
$$

this degeneracy point in a 3 -dimensional $k$-space becomes a source of the spatial divergence of the $U(1)$ magnetic field associated with these two bands;

$$
\nabla_{k} \cdot \overline{\mathcal{B}}^{\alpha}=\operatorname{sign}\left\{\operatorname{det} V^{\alpha}\right\} \delta^{3}\left(k-k^{\mathrm{mm}}\right) \equiv \rho_{\alpha}(k) .
$$

The sign of the magnetic charge is given by the asymptotic form of the effective 2 by 2 Hamiltonian around this degeneracy point;

$$
\hat{\mathrm{L}}^{(\alpha, \alpha+1)}(k) \simeq \sum_{\mu, \nu=x, y, z}\left(k_{\mu}-k_{\mu}^{\mathrm{mm}}\right) V_{\mu \nu}^{\alpha} \hat{\sigma}_{\nu}
$$
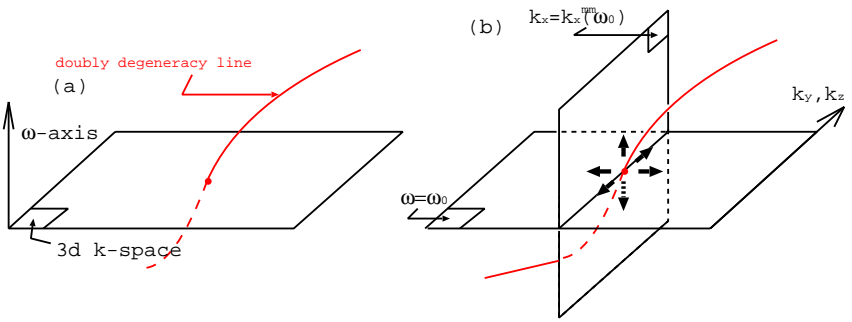

FIG. 6: (a) $L_{d, \alpha}$ and $L_{d, \alpha+1}$ are degenerated at a line in the dual $(3+1)$-dimensional space, which we dub as doubly degeneracy line (red curved line). The plane in this figure corresponds to a 3-dimensional space, while its complementary axis is specified by the four-th direction. (b) $k_{x}=k_{x}^{\mathrm{mm}}\left(\omega_{0}\right)$ plane and $\omega=\omega_{0}$ plane share a "magnetic charge" at $(\omega, k)=\left(\omega_{0}, k^{\mathrm{mm}}\left(\omega_{0}\right)\right)$. The relative sign between the charge viewed from the former and that from the latter is determined by the sign of the $x$-component of magnetic charge current (compare eq. (C1) and eq. (C5)).

where $\hat{\sigma}_{\mu}$ stands for the Pauli matrices ${ }^{33}$.

As was discussed in this paper, eigenvalues of the Lagrangian $\hat{L}$ in Fermi liquid acquire another argument $\omega$ in addition to the crystal momentum $k$. The doubly degeneracy point in a 3-dimensional space, however, is by construction stable against any four-th axis. Thus, along this $\omega$-direction, this degeneracy point forms a degeneracy line (see Fig. 6(a));

$$
L_{d, \alpha}\left(k^{\mathrm{mm}}(\omega), \omega\right) \equiv L_{d, \alpha+1}\left(k^{\mathrm{mm}}(\omega), \omega\right),
$$

Regarding $\omega$ as a sort of "time", one can therefore say that the $U(1)$ magnetic charge in 3-dimensional dual space is a conserved quantity. Corresponding to this conservation, we might as well introduce the $U(1)$ magnetic monopole current;

$$
j_{\alpha, \mu}^{\mathrm{mm}}(k, \omega) \equiv \operatorname{sign}\left\{\operatorname{det} V^{\alpha}\right\} \frac{d k_{\mu}^{\mathrm{mm}}}{d \omega} \delta^{3}\left(k-k^{\mathrm{mm}}(\omega)\right),
$$

so that its spatial divergence is balanced by the temporal derivative of the magnetic monopole density;

$$
\sum_{\mu=x, y, z} \partial_{k_{\mu}} j_{\alpha, \mu}^{\mathrm{mm}}+\partial_{\omega} \rho_{\alpha}(k, \omega) \equiv 0 .
$$

Then, in analogy to the Maxwell equation in a real space, we can introduce the dual version of "Ampere law" for the $U(1)$ electric field. Namely, the temporal derivative of the dual magnetic field and spatial rotation of the dual electric field is originated from this magnetic monopole current;

$$
\nabla_{k} \times \overline{\mathcal{E}}^{\alpha}+\partial_{\omega} \overline{\mathcal{B}}^{\alpha}=-j_{\alpha}^{\mathrm{mm}} .
$$

To see this law in a primitive way, one has only to consider the Gauss law in another 3-dimensional space, such as $k_{x}=$ constant plane, which crosses the doubly degeneracy line mentioned above (see Fig.6(b)). Namely, 
our $\omega-k_{y}-k_{z}$ space, say $k_{x}=k_{x}^{\mathrm{mm}}\left(\omega_{0}\right)$ plane, contain a doubly degeneracy point at

$$
\left(\omega, k_{y}, k_{z}\right)=\left(\omega_{0}, k_{y}^{\mathrm{mm}}\left(\omega_{0}\right), k_{z}^{\mathrm{mm}}\left(\omega_{0}\right)\right) .
$$

Therefore, generically, the doubly degeneracy at $k=$ $k^{\mathrm{mm}}\left(\omega_{0}\right)$ also becomes a source of the "magnetic field" defined in this 3 -dimensional space, which is now $\left(\overline{\mathcal{B}}_{x}^{\alpha},-\overline{\mathcal{E}}_{z}^{\alpha}, \overline{\mathcal{E}}_{y}^{\alpha}\right)$. To determine the sign of the corresponding "magnetic charge" viewed from this $\omega-k_{y}-k_{z}$ space, expand eq. (C2) with respect to $\omega$ around $\omega=\omega_{0}$,

$$
\begin{aligned}
\sum_{\mu, \nu=x, y, z}\left\{k_{\mu}-k_{\mu}^{\mathrm{mm}}(\omega)\right\} V_{\mu \nu}^{\alpha}(\omega) \hat{\sigma}_{\nu} \\
\quad \simeq\left[\omega-\omega_{0} k_{y}-k_{y}^{\mathrm{mm}}\left(\omega_{0}\right) k_{z}-k_{z}^{\mathrm{mm}}\left(\omega_{0}\right)\right] \cdot\left[\begin{array}{ccc}
-\frac{\partial k_{x}^{\mathrm{mm}}}{\partial \omega} & -\frac{\partial k_{y}^{\mathrm{mm}}}{\partial \omega} & -\frac{\partial k_{z}^{\mathrm{mm}}}{\partial \omega} \\
0 & 1 & 0 \\
0 & 0 & 1
\end{array}\right]_{\mid \omega=\omega_{0}} \cdot\left[V^{\alpha}\left(\omega_{0}\right)\right] \cdot\left[\begin{array}{c}
\hat{\sigma}_{x} \\
\hat{\sigma}_{y} \\
\hat{\sigma}_{z}
\end{array}\right] .
\end{aligned}
$$

where we imposed $k_{x}=k_{x}^{\mathrm{mm}}\left(\omega_{0}\right)$ in the right hand side, only to discuss the Gauss law in $k_{x}=$ constant plane. Namely, comparing this with eqs. (C1/C2), we obtain the Gauss law in this $\omega-k_{y}-k_{z}$ space;

$$
\begin{aligned}
\partial_{\omega} \overline{\mathcal{B}}_{x}^{\alpha}+ & \partial_{k_{y}}\left(-\overline{\mathcal{E}}_{z}^{\alpha}\right)+\partial_{k_{z}} \overline{\mathcal{E}}_{y}^{\alpha}=-\operatorname{sign}\left\{\operatorname{det} V^{\alpha} \frac{\partial k_{x}^{\mathrm{mm}}}{\partial \omega}\right\}_{\mid \omega=\omega_{0}} \\
& \times \delta\left(\omega-\omega_{0}\right) \delta\left(k_{y}-k_{y}^{\mathrm{mm}}\left(\omega_{0}\right)\right) \delta\left(k_{z}-k_{z}^{\mathrm{mm}}\left(\omega_{0}\right)\right) .(\mathrm{C} 5)
\end{aligned}
$$

By solving $\omega_{0}$ in favor of $k_{x}$, we can actually see that this equation is nothing but the $x$-component of eq. (C4).

\section{APPENDIX D: EXAMPLES - ELECTRON-PHONON COUPLING SYSTEMS -}

In this appendix, based on a specific model calculations, we will present the crude estimate of the dual version of $U(1)$ electric field. Basically, the electric component arises, in a perturbative calculation, from the frequency dependence of the self-energy. Moreover, we are interested in the hermitian part of the self-energy. This is analytically more difficult to extract than wellstudied anti-hermitian part. Namely, the latter involves an energy-conserving delta function, while the former takes over the corresponding principal integrals. As a result, a consideration for the case of the Coulomb interaction becomes quite complex, the frequency dependence arising only at two loops. We therefore consider instead the self-energy due to an electron-phonon interaction, for which we already have a non-trivial frequency dependence at one loop. Though we did not discuss the electron-phonon interaction explicitly in our derivations, the considerations there still apply to this problem. Namely, our formulations are clearly free from the specific origin of the self-energy, provided that its anti-hermitian part can be negligible at low frequency region.

As a simplest model, we considered the 2D Rashba model defined on a square lattice, subjected to an exter- nal magnetic field along the $z$-direction;

$$
\begin{aligned}
\mathcal{H}_{0}= & \frac{t}{2} \sum_{\langle j m\rangle, \alpha} \psi_{j, \alpha}^{\dagger} \psi_{m, \alpha}+h_{z} \sum_{j, \alpha, \beta} \psi_{j, \alpha}^{\dagger}\left[\hat{\tau}_{z}\right]_{\alpha, \beta} \psi_{j, \beta} \\
& -a \sum_{j} \sum_{\mu, \nu=x, y} \frac{i \epsilon_{\mu \nu}}{2}\left(\psi_{j+e_{\mu}, \alpha}^{\dagger}\left[\hat{\tau}_{\nu}\right]_{\alpha, \beta} \psi_{j, \beta}-\text { h.c. }\right) .
\end{aligned}
$$

We employ the lattice-regularized model, since the hermitian part of the self-energy usually depends on the ultraviolet cut-off. Namely, $\hat{\sigma}$ obtained from the Matsubara Green function is composed of a principal integral with respect to momentum, which shows a ultraviolet logarithmic divergence in a 2D model (see for example eq.(D1)). The above Hamiltonian, however, can be referred as a "Rashba" model, in a sense that, when Fourier transformed, it reproduces the same energy dispersion as the standard (i.e. continuum) Rashba model, around the $\Gamma$ point;

$$
\begin{aligned}
\mathcal{H}_{0} \equiv & \sum_{\alpha, \beta} \sum_{k} \psi_{\alpha}^{\dagger}(k)\left[\hat{H}_{0}(k)\right]_{\alpha, \beta} \psi_{\beta}(k), \\
\hat{H}_{0}(k) \equiv- & t\left(\cos k_{x}+\cos k_{y}-2\right) \hat{1} \\
& \quad+h_{z} \hat{\tau}_{z}+a \sin k_{x} \hat{\tau}_{y}-a \sin k_{y} \hat{\tau}_{x},
\end{aligned}
$$

with $\psi_{\alpha}^{\dagger}(k) \equiv \frac{1}{\sqrt{N}} \sum_{j} e^{i k \cdot j} \psi_{\alpha}^{\dagger}(j)$.

A finite magnetic field $h_{z}$ lifts the band degeneracy at $k=(0,0),(\pi, 0),(0, \pi)$ and $(\pi, \pi)$;

$$
\mathrm{e}_{ \pm, k}=-t\left(\cos k_{x}+\cos k_{y}-2\right) \pm\left|\lambda_{k}\right|
$$

where $\left|\lambda_{k}\right| \equiv \sqrt{h_{z}^{2}+a^{2}\left(\sin ^{2} k_{x}+\sin ^{2} k_{y}\right)}$. Concomitantly, the magnetic Bloch wavefunctions for these two energy bands also acquire finite $z$-components of spin in the presence of $h_{z}$;

$$
\left|\mathbf{u}_{k}^{+}\right\rangle=\left[\begin{array}{c}
\cos \left(\frac{\theta_{k}}{2}\right) \\
\sin \left(\frac{\theta_{k}}{2}\right) e^{i \varphi_{k}}
\end{array}\right],\left|\mathbf{u}_{k}^{-}\right\rangle=\left[\begin{array}{c}
-\sin \left(\frac{\theta_{k}}{2}\right) e^{-i \varphi_{k}} \\
\cos \left(\frac{\theta_{k}}{2}\right)
\end{array}\right],
$$


where $\theta_{k}$ and $\varphi_{k}$ are defined as follows;

$$
\begin{aligned}
\left(\cos \theta_{k}, \sin \theta_{k}\right) & \equiv \frac{1}{\left|\lambda_{k}\right|}\left(h_{z}, a \sqrt{\sin ^{2} k_{x}+\sin ^{2} k_{y}}\right) \\
\left(\cos \varphi_{k}, \sin \varphi_{k}\right) & \equiv \frac{1}{\sqrt{\sin ^{2} k_{x}+\sin ^{2} k_{y}}}\left(\sin k_{x}, \sin k_{y}\right)
\end{aligned}
$$

We have introduced the magnetic field, because, in a time-reversal symmetric system, the a.e.f.-contribution to the anomalous Hall effect (AHE) as well as that of the dual magnetic field (a.m.f.) always vanishes. Namely, the electric field is time-reversally "even" in a $T$-invariant system;

$$
\mathcal{E}^{\alpha}(k)=\mathcal{E}^{\alpha}(-k),
$$

while the quasi-particle velocity is T-reversal "odd";

$$
\mathbf{v}_{\alpha}(k)=-\mathbf{v}_{\alpha}(-k) .
$$

Thus, $\mathcal{E}^{\alpha}(k) \times \mathbf{v}_{\alpha}(k)$ would vanish after the $k$-integration in a $T$-invariant system.

As for "many-body" effects, we consider an electronphonon coupled Hamiltonian, in which the Einstein phonon having constant mass $\omega_{0}>0$ interacts with this Rashba fermion;

$$
\begin{aligned}
& \mathcal{H}_{\mathrm{ph}}+\mathcal{H}_{\mathrm{ep}}= \\
& \sum_{q} \omega_{0} b_{q}^{\dagger} b_{q}+\sum_{k, q, \alpha, \beta} \psi_{\alpha}^{\dagger}(k+q)\left[\hat{g}_{q}\right]_{\alpha, \beta} \psi_{\beta}(k) \cdot\left(b_{q}+b_{-q}^{\dagger}\right) .
\end{aligned}
$$

The hermiteness clearly requires $\hat{g}_{q}^{\dagger}=\hat{g}_{-q}$ in general. We take the simplest possible forms for this e-p coupling constant: $\hat{g}_{q} \simeq \hat{g}_{q=0}$, and $\hat{g}_{q=0} \simeq g \cdot \hat{1}$. Even employing this over-simplified coupling form, we already find a substantial magnitude of the electric field, as shown below.

The lowest order (imaginary-time) self-energy associated with this electron-phonon system is 2nd order in $g$

$$
\begin{aligned}
& \hat{\Sigma}\left(k, i \omega_{m}\right)=\frac{g^{2}}{N} \sum_{q} \sum_{\gamma= \pm}\left|\mathrm{u}_{k+q}^{\gamma}\right\rangle\left\langle\mathrm{u}_{k+q}^{\gamma}\right| \\
& \times\left\{\frac{n_{\mathrm{b}}\left(\omega_{0}\right)+n_{\mathrm{f}}\left(\mathrm{e}_{\gamma, k+q}\right)}{i \omega_{m}+\omega_{0}-\mathrm{e}_{\gamma, k+q}}+\frac{n_{\mathrm{b}}\left(\omega_{0}\right)+1-n_{\mathrm{f}}\left(\mathrm{e}_{\gamma, k+q}\right)}{i \omega_{m}-\omega_{0}-\mathrm{e}_{\gamma, k+q}}\right\},
\end{aligned}
$$

where $n_{\mathrm{b}}(\omega)=\frac{1}{e^{\beta \omega}-1}$ denotes the Bose distribution function.

At equilibrium, the life-time part of the self-energy and hermitian part of the self-energy $\hat{\sigma}$ are analytically continued from this imaginary-time one (see also eqs. (14-15);

$$
\begin{aligned}
& \Gamma_{\alpha \beta}(k, \omega) \equiv i \Sigma_{\alpha \beta}\left(k, i \omega_{m}=\omega+i|\delta|\right) \\
& -i \Sigma_{\alpha \beta}\left(k, i \omega_{m}=\omega-i|\delta|\right), \\
& \sigma_{\alpha \beta}(k, \omega) \equiv \frac{1}{2}\left\{\Sigma_{\alpha \beta}\left(k, i \omega_{m}=\omega+i|\delta|\right)\right. \\
& \left.+\Sigma_{\alpha \beta}\left(k, i \omega_{m}=\omega-i|\delta|\right)\right\} .
\end{aligned}
$$

The life-time term clearly becomes zero at $T=0$ and $\omega \simeq \mu$. Namely its integral region over the internal line is exponentially small:

$$
\begin{aligned}
\left(n_{\mathrm{b}}\left(\omega_{0}\right)+n_{\mathrm{f}}(\mathrm{e})\right) \cdot \delta\left(\omega+\omega_{0}-\mathrm{e}\right) & \simeq 0 \\
\left(n_{\mathrm{b}}\left(\omega_{0}\right)+1-n_{\mathrm{f}}(\mathrm{e})\right) \cdot \delta\left(\omega-\omega_{0}-\mathrm{e}\right) & \simeq 0
\end{aligned}
$$

when $|\omega-\mu| \ll \omega_{0}$ at $T \rightarrow 0$. On the other hand, the hermitian part of the self-energy, which is made up of the principal integral, remains finite even at $T=0$;

$$
\begin{aligned}
\hat{\sigma}(k, \omega)=g^{2} & \sum_{\gamma= \pm}\left\{\int_{\mathrm{e}_{\gamma, k^{\prime}} \leq \mu} \mathcal{P} \frac{\left|\mathrm{u}_{k^{\prime}}^{\gamma}\right\rangle\left\langle\mathrm{u}_{k^{\prime}}^{\gamma}\right|}{\omega+\omega_{0}-\mathrm{e}_{\gamma, k^{\prime}}}\right. \\
& \left.+\int_{\mathrm{e}_{\gamma, k^{\prime}} \geq \mu} \mathcal{P} \frac{\left|\mathrm{u}_{k^{\prime}}^{\gamma}\right\rangle\left\langle\mathrm{u}_{k^{\prime}}^{\gamma}\right|}{\omega-\omega_{0}-\mathrm{e}_{\gamma, k^{\prime}}}\right\} \frac{d k^{\prime}}{(2 \pi)^{2}}
\end{aligned}
$$

where we introduced the new integral variable $k^{\prime} \equiv k+q$.

When integrated over $k^{\prime}$, the off-diagonal elements of the 2 by 2 matrix $\left|\mathbf{u}_{k^{\prime}}^{\gamma}\right\rangle\left\langle\mathbf{u}_{k^{\prime}}^{\gamma}\right|$ vanish. Namely, they are always odd functions of $k_{x}^{\prime}$ or $k_{y}^{\prime}$, while $\mathrm{e}_{\gamma, k^{\prime}}$ is even;

$$
\begin{aligned}
\left|\mathbf{u}_{k^{\prime}}^{\gamma}\right\rangle\left\langle\mathbf{u}_{k^{\prime}}^{\gamma}\right| & =\frac{1}{2} \hat{1}+\frac{\operatorname{sign} \gamma}{2}\left(\cos \theta_{k^{\prime}} \hat{\tau}_{z}\right. \\
& \left.+\sin \theta_{k^{\prime}} \cos \varphi_{k^{\prime}} \hat{\tau}_{x}-\sin \theta_{k^{\prime}} \sin \varphi_{k^{\prime}} \hat{\tau}_{y}\right) .
\end{aligned}
$$

Accordingly, eq.(D1) becomes diagonal;

$$
\begin{aligned}
& \hat{\sigma}(k, \omega)=\{\cdots\} \cdot \hat{1}+\frac{g^{2}}{2} \sum_{\gamma= \pm} \\
& \left\{\int_{\epsilon_{k^{\prime}, \gamma} \leq \mu} \mathcal{P} \cdot \frac{\operatorname{sign} \gamma \cos \theta_{k^{\prime}}}{\omega+\omega_{0}-\epsilon_{k^{\prime}, \gamma}} \frac{d k^{\prime}}{(2 \pi)^{2}}\right. \\
& \left.+\int_{\epsilon_{k^{\prime}, \gamma} \geq \mu} \mathcal{P} \cdot \frac{\operatorname{sign} \gamma \cos \theta_{k^{\prime}}}{\omega-\omega_{0}-\epsilon_{k^{\prime}, \gamma}} \frac{d k^{\prime}}{(2 \pi)^{2}}\right\} \hat{\tau}_{z}, \\
& \equiv S_{0}(\omega) \cdot \hat{1}+S_{1}(\omega) \hat{\tau}_{z} .
\end{aligned}
$$

The scalar function $S_{1}(\omega)$ is always a smooth function at $|\omega-\mu| \ll \omega_{0}$. When this self-energy diagonalized in combination with $\hat{H}_{0}(k)$, a non-zero derivative of $S_{1}(\omega)$ with respect to $\omega$ becomes indispensable for a finite a.e.f., which we will see below.

In a $2 \mathrm{D}$ model, we generally have 2-component electric field and a single-component magnetic field;

$$
\begin{aligned}
& \left(-\mathcal{E}_{y}^{\gamma}, \mathcal{E}_{x}^{\gamma}, \mathcal{B}_{z}^{\gamma}\right) \equiv \nabla \times \mathcal{A}^{\gamma}, \\
& \mathcal{A}_{\mu}^{\gamma} \equiv i\left\langle u_{k, \omega}^{\gamma} \mid \partial_{\mu} u_{k, \omega}^{\gamma}\right\rangle
\end{aligned}
$$

with $\mu=\left(k_{x}, k_{y}, \omega\right)$. Note that $\left|u_{k, \omega}^{\gamma}\right\rangle$ diagonalizes the $\hat{\mathrm{L}}(k, \omega)=\hat{H}_{0}(k)+\hat{\sigma}(\omega)$. For any 2-band model, the Lagrangian can be decomposed in terms of the Pauli matrices;

$$
\hat{\mathrm{L}}(k, \omega)=\sum_{\mu=x, y, z} N_{\mu}(k, \omega) \cdot \hat{\tau}_{\mu}+\text { const. }
$$

where $N$ now reads $\left(-a \sin k_{y}, a \sin k_{x}, h_{z}+S_{1}(\omega)\right)$. Then, one readily see that the dual fields are identified as the 
solid angle subtended by this normalized vector $\hat{N} \equiv$ $N /|N|$. For example, its spatial component reads

$$
\begin{aligned}
\mathcal{B}_{z}^{\gamma} & =-\frac{\operatorname{sign} \gamma}{2}\left(\nabla_{k_{x}} \hat{N} \times \nabla_{k_{y}} \hat{N}\right) \cdot \hat{N}, \\
& =\frac{\operatorname{sign} \gamma \cdot N_{z}}{2|N|^{3}} \cdot \frac{\partial N_{y}}{\partial k_{x}} \frac{\partial N_{x}}{\partial k_{y}} \\
& =-\frac{\operatorname{sign} \gamma \cdot a^{2} N_{z}}{2|N|^{3}} \cos k_{x} \cos k_{y},
\end{aligned}
$$

where the band index $\gamma= \pm$. Similarly, the temporal components are given as follows;

$$
\begin{aligned}
\left(\mathcal{E}_{x}^{\gamma}, \mathcal{E}_{y}^{\gamma}\right) & =-\frac{\operatorname{sign} \gamma}{2|N|^{3}} \frac{\partial S_{1}}{\partial \omega} \hat{z} \cdot\left(\frac{\partial N}{\partial k_{x}} \times N, \frac{\partial N}{\partial k_{y}} \times N\right) \\
& =-\frac{\operatorname{sign} \gamma \cdot a^{2}}{2|N|^{3}} \frac{\partial S_{1}}{\partial \omega}\left(\cos k_{x} \sin k_{y},-\cos k_{y} \sin k_{x}\right), \\
& \equiv \operatorname{sign} \gamma \cdot\left|E_{k}\right|\left(\cos k_{x} \sin k_{y},-\cos k_{y} \sin k_{x}\right),
\end{aligned}
$$

which clearly indicates that a finite $\frac{\partial S_{1}}{\partial \omega}$ is the essential origin of the electric fields. With the lattice constant $a_{\text {lattice }}$ being explicit, the magnitude of the dual electric field estimated on a Fermi surface, i.e. $\omega=\mu$, is given as follows;

$$
\begin{aligned}
& \left|E_{k}\right|_{\mid \omega=\mu}=\frac{\mathrm{a}_{\text {lattice }}}{t} \cdot \frac{\bar{a}^{2} \bar{g}^{2} \bar{h}_{z}}{2\left|\bar{N}_{k}\right|^{3}} \times f, \\
& f=\sum_{\gamma= \pm}\left\{\int_{\overline{\mathrm{e}}_{\gamma, q} \leq 0} \frac{\operatorname{sign} \gamma}{\bar{\Delta}_{q} \cdot\left(\bar{\omega}_{0}-\overline{\mathrm{e}}_{\gamma, q}\right)^{2}}\right. \\
& \left.+\int_{\overline{\mathrm{e}}_{\gamma, q} \geq 0} \frac{\operatorname{sign} \gamma}{\bar{\Delta}_{q} \cdot\left(\bar{\omega}_{0}+\overline{\mathrm{e}}_{\gamma, q}\right)^{2}}\right\} \frac{d q}{(2 \pi)^{2}},
\end{aligned}
$$

where $\bar{\Delta}_{q}$ denotes the direct band gap at $q$-point, measured with the transfer integral " $t$ " being an energy unit; $\bar{\Delta}_{q} \equiv 2\left|\lambda_{q}\right| / t$. In a same sense, we also made it dimensionless, the Rashba coupling energy, e-p coupling energy, Zeeman energy, phonon energy and band dispersion, like $\bar{a}, \bar{g}, \bar{h}_{z}, \bar{\omega}_{0}$ and $\overline{\mathrm{e}}_{\gamma, q} \equiv\left(\mathrm{e}_{\gamma, q}-\mu\right) / t$ respectively. In terms of these, the other dimensionless function of $k$, i.e. $\left|\bar{N}_{k}\right|$, reads;

$$
\begin{aligned}
\left|\bar{N}_{k}\right|= & \sqrt{\bar{a}^{2}\left(\sin ^{2} k_{y}+\sin ^{2} k_{x}\right)+\bar{h}_{z}^{2}\left(1+\bar{S}_{1}\right)^{2}}, \\
\bar{S}_{1}= & \bar{g}^{2} \sum_{\gamma= \pm}\left\{\int_{\overline{\mathrm{e}}_{\gamma, q} \leq 0} \frac{\operatorname{sign} \gamma}{\bar{\Delta}_{q} \cdot\left(\bar{\omega}_{0}-\overline{\mathrm{e}}_{\gamma, q}\right)}\right. \\
& \left.-\int_{\overline{\mathrm{e}}_{\gamma, q} \geq 0} \frac{\operatorname{sign} \gamma}{\bar{\Delta}_{q} \cdot\left(\bar{\omega}_{0}+\overline{\mathrm{e}}_{\gamma, q}\right)}\right\} \frac{d q}{(2 \pi)^{2}} .
\end{aligned}
$$

The expression eq. (D5) clearly demonstrates that the magnitude of the electric field is finite only in the presence of the applied magnetic field $h_{z}$ and Rashba coupling $a$. It is also proportional to the dimensionless factor, i.e. $f$, whose value depends on a specific shape of the upper band and lower band in $k$-space. From its form, however, there is no reason that this real-valued factor always has to reduce identically to zero. In fact, a simple numerical

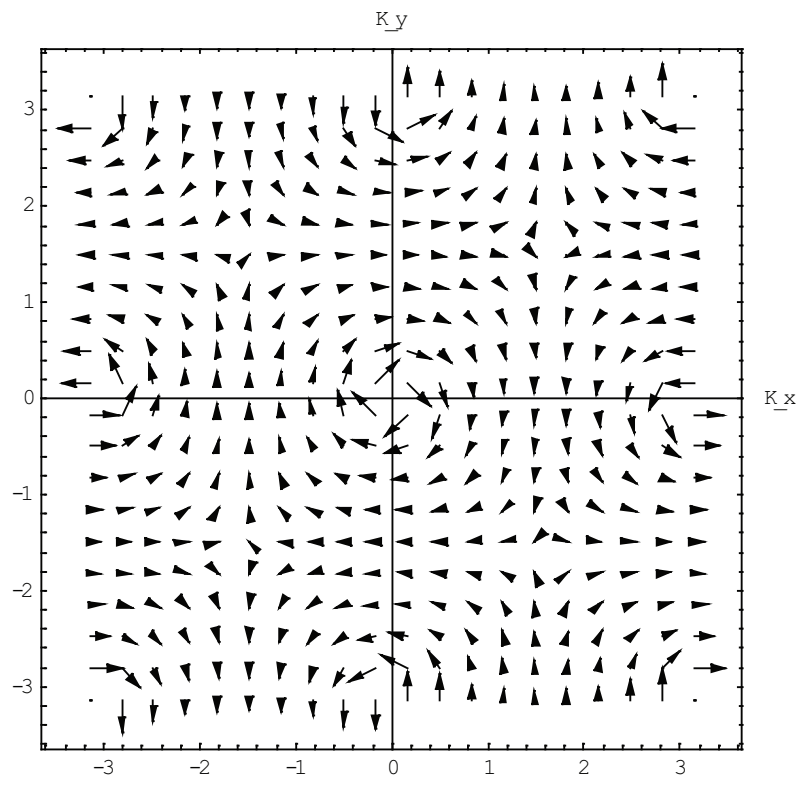

FIG. 7: A distribution of $\left(\mathcal{E}_{x}^{-}, \mathcal{E}_{y}^{-}\right)$estimated on a Fermi surface, i.e. $\omega=\mu . \quad \bar{a}=1.0, \bar{g}=2.0, \bar{h}_{z}=0.5, \bar{\omega}_{0}=0.5$ and $\mu=0$.

estimation shows that $f=-0.31$ and $\bar{S}_{1}=-0.24$, in the case of $\bar{a}=1.0, \bar{g}=2.0, \bar{h}_{z}=0.5, \mu=0$ with $\bar{\omega}_{0}=0.5$. Observing these numerical factors, the magnitude of the a.e.f. is estimated as follows;

$$
\left|\mathcal{E}^{\alpha}\right| \simeq 10^{-1} \times \frac{\text { alattice }}{t} .
$$

The distribution of the a.e.f. has a 4 vortex structure within the unit cell $[-\pi, \pi] \times[-\pi, \pi]$ (see Fig. 17). These vortices reflect the 4 band-crossing points located at $\left(k_{x}, k_{y}, \omega\right)=\left(0,0, \omega_{1}\right),\left( \pm \pi, \pm \pi, \omega_{1}\right),\left(0, \pm \pi, \omega_{1}\right)$ and $\left( \pm \pi, 0, \omega_{1}\right)$ with $\omega_{1}$ defined as follows;

$$
h_{z}+S_{1}\left(\omega_{1}\right)=0 .
$$

Namely, $\left(-\mathcal{E}_{y}^{-}, \mathcal{E}_{x}^{-}, \mathcal{B}_{z}^{-}\right)$has sources $(2 \pi$ "charges" $)$ from the former two points, while it has sinks ( $-2 \pi$ "charges") at the latter two.

The many-body correction to the anomalous velocity is given by the outer product between the a.e.f. and the quasi-particle velocity, i.e., $\left(\mathcal{E}_{k}^{\alpha} \times \mathbf{v}_{\alpha}\right)_{z}$. Thus, taking $\left|\mathbf{v}_{\alpha}\right|$ to be alattice $\cdot t$, we find it of the order of $10^{-1} \times \mathrm{a}_{\text {lattice }}^{2}$. The bare contribution of the anomalous velocity on a Fermi surface can be directly estimated from eq.(D4); $\mathcal{B}_{z}^{\alpha} \simeq$ $1.0 \times \mathrm{a}_{\text {lattice }}^{2}$. Observing these two quantities, we can then insist that the electric-field contribution becomes almost at the same order of this bare contribution and not negligible even in this oversimplified model. 
* Present address: Division of Materials Physics, Department of Physical Science, Graduate School of Engineering Science, Osaka University; Electronic address: rshindou@postman.riken.jp

1 E. Fradkin, Field Theories of Condensed Matter Systems (Addison-Wesley, Redwood City, 1991)

2 A. H. Castro Neto, P. Pujol and E. Fradkin, Physical Review $\mathrm{B}, \mathbf{7 4}$ 024302, (2006).

3 D. L. Bergman, G. A. Fiete and L. Balents, Physical Review B, 73 134402, (2006).

4 O. I. Motrunich and T. Senthil, Physical Review B, 71 125102, (2005).

5 G. Sundaram and Q. Niu, Physical Review B, 59 14915, (1999)

6 S. Murakami, N. Nagaosa and S. C. Zhang, Science 301 1348, (2003).

7 M. Onoda, S. Murakami, N. Nagaosa, Phys. Rev. E 74 066610, (2006).

${ }^{8}$ D. Culcer, Y. Yao and Q. Niu Physical Review B 72 085110, (2005).

${ }^{9}$ K. Y. Bliokh and Y. P. Bliokh Annals of Physics 319 13, (2005).

10 R. Shindou and K-i Imura Nuclear Physics B 720 [FS] 399, (2005).

11 Y. Kato, R. C. Myers, A. C. Gossard and D. D. Awschalom, Nature 429 50, (2004).

12 J. Sinova, D. Culcer, Q. Niu, N. A. Sinitsyn, T. Jungwirth, and A. H. MacDonald, Physical Review Letters, 92 126603, (2004).

13 Y. G. Yao, L. Kleinman, A. H. MacDonald, J. Sinova, T. Jungwirth, D. S. Wang, E. G. Wang and Q. Niu, Physical Review Letters 92 037204, (2004).

14 J. Wunderlich, B. Kaestner, J. Sinova and T. Jungwirth, Physical Review Letters, 94 047204, (2005).

15 W. L. Lee, S. Watauchi, V. L. Miller, R. J. Cava and N. P. Ong Science 303 1647, (2004).

16 S. O. Valenzuela and M. Tinkham, Nature, 442 176, (2006).

17 For example, see http://online.kitp.ucsb.edu/online/spintr06/

18 C. Day, Physics Today, 58 17, (2005)

19 J. P. Hu, D. W. Shen and D. L. Feng Physical Review B, 73 085325, (2006)

20 J. Shi and Q. Niu, cond-mat/0601531

21 F. D. M. Haldane, Physical Review Letters, 93206602 , (2004)

22 F. D. M. Haldane, Proceedings of the International School of Physics "Enrico Fermi", Course CXXI "Perspectives in Many-Particle Physics", eds. R. A. Broglia and J. R. Schrieffer (North-Holland, Amseterdam 1994) 5-29

23 see http://wwwphy.princeton.edu/ duncan/research.html

24 A similar result as we will obtain in $U(1)$ FL was also obtained by using different arguments in the context of non-interacting $U(1)$ Fermi system; D. Xiao, J. R. Shi and Q. Niu, Physical Review Letters, 95 137204, (2005)

25 L. P. Kadanoff and G. Baym, Quantum Statistical Mechan- ics (Perseus Press, Cambridge, 1989)

26 G. D. Mahan, Many-Particle Physics (Kluwer Academic/Plenum Publishers, New York, 2000)

27 J. Rammer and H. Smith, Review of Modern Physics, 58 $323,(1986)$

28 Note that we have neglected the following terms which is of the order of $\mathcal{O}\left(\lambda^{3}\right)$;

$$
\left[\left[\hat{\epsilon}_{\alpha}, \hat{\Omega}_{X_{j} Q_{j}}^{\alpha}\right]_{+}, \hat{A}_{\alpha}\right]_{-} \simeq \mathcal{O}\left(\lambda_{1}^{2} \lambda_{2}, \lambda_{1} \lambda_{2}^{2}\right) .
$$

Namely, $\hat{A}_{\alpha}$ being unit matrix at equilibrium, this commutator is 3rd order in $\lambda$.

29 E. M. Landau and L. P. Lifshitz, Statistical Physics Part.2 (Butterworth-Heinemann, Oxford, 2002)

30 R. Shindou and L. Balents, Physical Review Letters, 97 216601, (2006)

31 K. Ishikawa and T. Matsuyama, Zeitschrift Fur Physik C - Particles and Fields, 33 41, (1986); Nuclear Physics B, $\mathbf{2 8 0}$ [FS18] 523, (1987).

32 Note that the $\sigma, \mathrm{b}$ and $G_{0}^{-1}-\hat{\Sigma}^{\mathrm{HF}}$ observe the followings;

$$
\begin{aligned}
& \sigma\left(1,1^{\prime}\right)^{*} \equiv \sigma\left(1^{\prime}, 1\right), \mathrm{b}\left(1,1^{\prime}\right)^{*} \equiv \mathrm{b}\left(1^{\prime}, 1\right) \\
& \left(G_{0}^{-1}-\hat{\Sigma}^{\mathrm{HF}}\right)\left(1,1^{\prime}\right)^{*} \equiv\left(G_{0}^{-1}-\hat{\Sigma}^{\mathrm{HF}}\right)\left(1^{\prime}, 1\right),
\end{aligned}
$$

while

$$
\begin{aligned}
& \mathrm{g}^{<(>)}\left(1,1^{\prime}\right)^{*} \equiv-\mathrm{g}^{<(>)}\left(1^{\prime}, 1\right) \\
& \Sigma_{\mathrm{c}}^{<(>)}\left(1,1^{\prime}\right)^{*} \equiv-\Sigma_{\mathrm{c}}^{<(>)}\left(1^{\prime}, 1\right)
\end{aligned}
$$

${ }^{33}$ M. V. Berry, Proc. Roy. Soc. London A 392, 45 (1984)

34 As in the standard literature ${ }^{25}$, we took the following conventions of the Fourier transformation of lesser/greater green function/collisional self energy with respect to the frequency;

$$
\begin{aligned}
& \mathrm{g}^{<}(\omega)=-i \int_{-\infty}^{\infty} e^{i \omega t} \mathrm{~g}^{<}(t) d t \\
& \mathrm{~g}^{>}(\omega)=i \int_{-\infty}^{\infty} e^{i \omega t} \mathrm{~g}^{>}(t) d t \\
& \Sigma_{\mathrm{c}}^{<}(\omega)=-i \int_{-\infty}^{\infty} e^{i \omega t} \Sigma_{\mathrm{c}}^{<}(t) d t \\
& \Sigma_{\mathrm{c}}^{>}(\omega)=i \int_{-\infty}^{\infty} e^{i \omega t} \Sigma_{\mathrm{c}}^{>}(t) d t
\end{aligned}
$$

only to obtain the relation between the spectral function and green function given below;

$$
\mathrm{A}(\omega) \equiv \int_{-\infty}^{\infty} e^{i \omega t} \mathrm{~A}(t) d t=\mathrm{g}^{>}(\omega)+\mathrm{g}^{<}(\omega)
$$

(see also eq. (17)) 